\title{
TÉCNICAS DE CONTROL NO LINEAL EN SISTEMAS ELECTRÓNICOS DE POTENCIA
}

Jorge Alberto SOLSONA

Tesis presentada para el grado de

DOCTOR EN INGENIERIA

Depto. de Electrotecnia, Facultad de Ingeniería, U.N.L.P., Abril, 1995 


\section{Agradecimientos}

Deseo agradecer a mis compañeros de trabajo por haber hecho del LEICI un lugar de convivencia agradable, donde he podido desarrollarme sin limitación alguna. También, quiero agradecerles por lo que aportaron a mi crecimiento como persona. De alguna manera, sus enseñanzas se encuentran reflejadas en este trabajo.

Quiero agradecer a Ricardo Mantz y Eugenio Tacconi, además, el beneficio que obtuve de su condescendencia y altruísmo.

Ex profeso he dejado para el final a Carlos Muravchik; a quién quiero agradecer, pues su apoyo incondicional, en cuestiones científicas y no científicas, ha actuado como la piedra basal de este trabajo. 


\section{Índice}

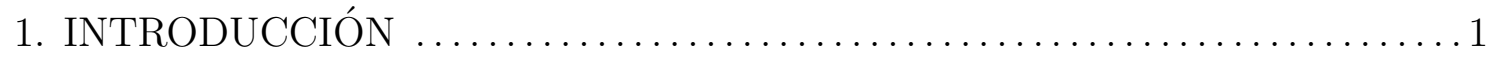

1.1 Perspectiva histórica y motivación $\ldots \ldots \ldots \ldots \ldots \ldots \ldots \ldots \ldots \ldots \ldots \ldots \ldots \ldots \ldots \ldots$

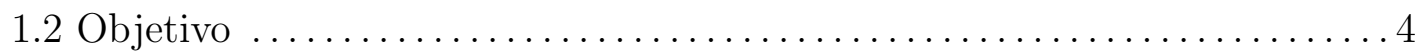

1.3 Observadores no lineales y accionamientos eléctricos $\ldots \ldots \ldots \ldots \ldots \ldots 5$

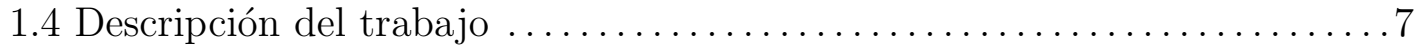

2. MODELO GENERAL DEL ACCIONAMIENTO $\ldots \ldots \ldots \ldots \ldots \ldots \ldots \ldots \ldots \ldots$

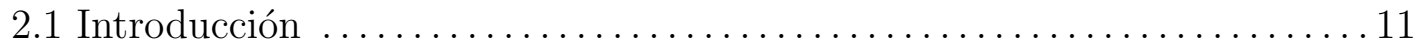

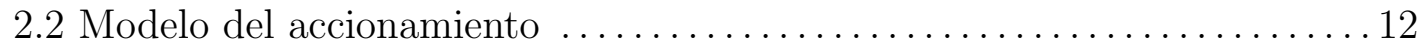

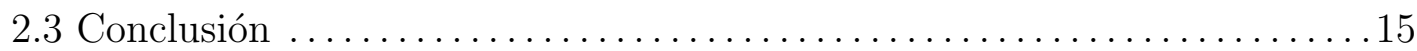

3. OBSERVADORES NO LINEALES $\ldots \ldots \ldots \ldots \ldots \ldots \ldots \ldots \ldots \ldots \ldots \ldots \ldots \ldots \ldots \ldots$

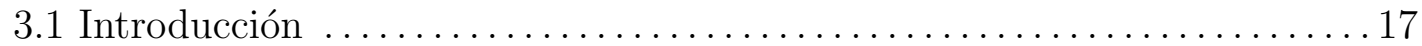

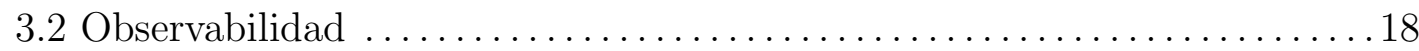

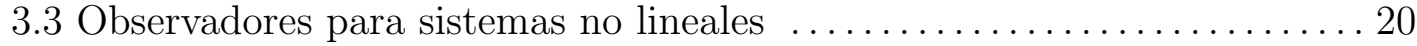

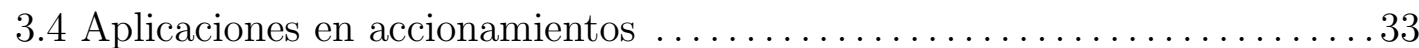

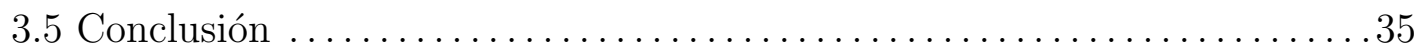

4. OBSERVADORES DE ORDEN REDUCIDO $\ldots \ldots \ldots \ldots \ldots \ldots \ldots \ldots \ldots \ldots$

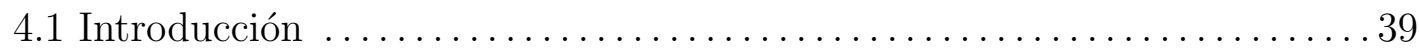

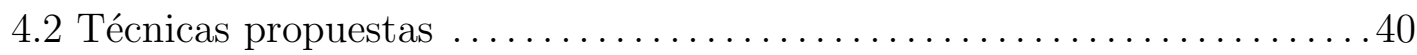

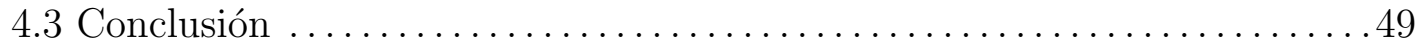

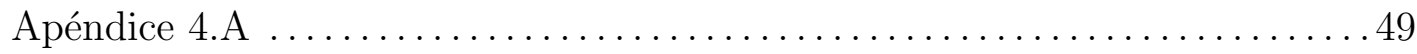

5. APLICACIÓN AL MRC .................................... 51

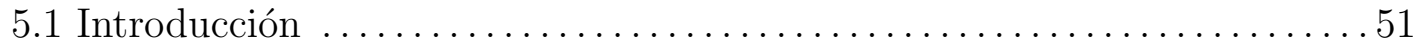




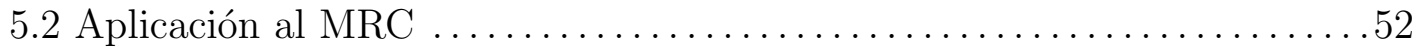

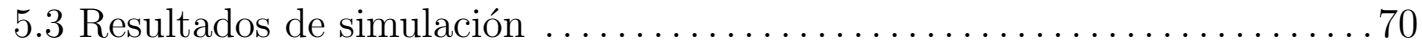

5.4 Conclusión ...............................................90

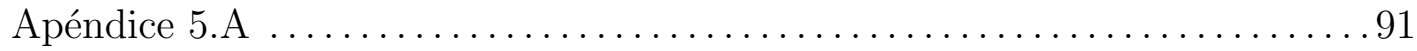

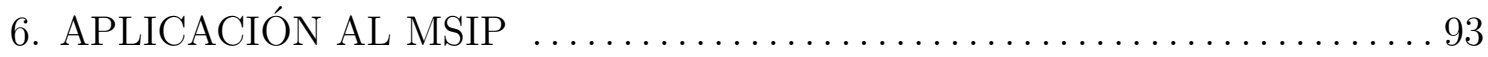

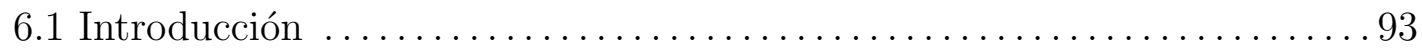

6.2 Aplicación al MSIP .................................... 94

6.3 Resultados de simulación .................................... 102

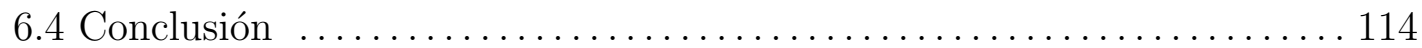

7. CONTROL DE VELOCIDAD DEL MSIP $\ldots \ldots \ldots \ldots \ldots \ldots \ldots \ldots \ldots \ldots \ldots \ldots$

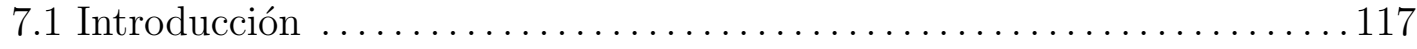

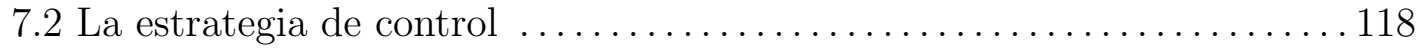

7.3 Control de velocidad sin sensores mecánicos . . . . . . . . . . . . . . . . 121

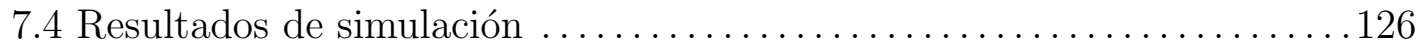

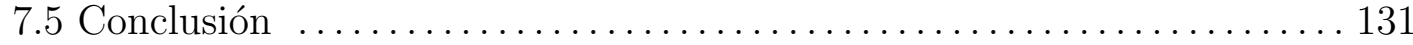

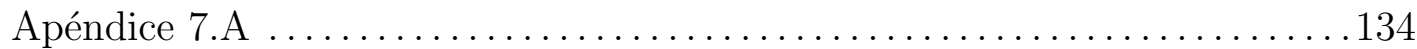

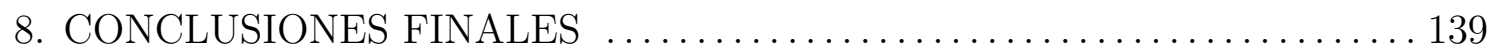

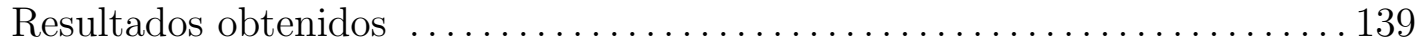

Sugerencias para futuros desarrollos . . . . . . . . . . . . . . . . . . . . . . 140

BIBLIOGRAFIA ........................................... 143 


\section{Capítulo 1}

\section{Introducción}

\subsection{Perspectiva histórica y motivación}

En los últimos años, el campo de los sistemas electrónicos de potencia, en particular los accionamientos eléctricos para uso industrial, fue mutando velozmente debido al avance en el desarrollo de dispositivos electrónicos de potencia, circuitos integrados y microprocesadores. Este avance, ha permitido la construcción de convertidores de relativamente reducido tamaño y bajo costo.

Esto ha impactado sobremanera en el campo de los accionamientos eléctricos, dando lugar a la mejora del desempeño y costo efectivo de los accionamientos de corriente alterna. En éstos, cada vez es mayor el grado de sofisticación de estrategias de control empleadas; no importa que las máquinas sean los tradicionales motores de inducción y sincrónico o los no tan tradicionales, pero de incipiente desarrollo, motores de continua sin escobillas y motores de paso [56], [82], [85]. En ciertas aplicaciones, por ejemplo, los trenes de laminación, el control numérico de máquinas herramienta, los controles de movimiento (manipuladores robóticos), en las que se requiere una velocidad variable, o un control de cupla o posición, era tradicional el uso de motores de corriente continua, pues éstos presentan características que facilitan el diseño de la estrategia de control. Sin embargo, presentan la dificultad de emplear escobillas para la conmutación. Esto trae aparejado una serie de inconvenientes en cuanto a confibialidad y mantenimiento. Principalmente por esta 
razón, actualmente, se evalúa el reemplazo, o el mismo ya se ha producido, de los motores de continua por accionamientos que utilizan motores de alterna [38], [69], [103]. Debido a que es inherente a la manera de funcionar de un accionamiento que utiliza este tipo de motores, el comando de las llaves del convertidor debe ser sincronizado con la posición del rotor del motor. Además, en los casos más complejos, cuando el o los motores participan como accionamientos de un sistema de manufactura muy agregado, los objetivos de control fijados para su operación suelen ser los más exigentes. Se puede decir que por razones de funcionamiento y para la implementación de las estrategias de control se requerirá del conocimiento de la posición y velocidad con que se mueve el rotor del motor. Una manera de obtener esta información es empleando sensores adecuados de posición y velocidad angular (codificadores angulares, taquigeneradores, por ejemplo). Si bien existe una gran variedad de sensores de dichas magnitudes, los más difundidos en la industria suelen ser de características mecánicas/ ópticas. Las ventajas de su uso derivan de que miden directamente las variables de interés. Sin embargo, su utilización presenta una serie de inconvenientes en el ámbito industrial.

Para evitar el empleo de sensores mecánicos / ópticos se puede utilizar un observador de estado. Éste permite, estimar la posición y la velocidad a partir de la medición de las tensiones y corrientes (magnitudes eléctricas) aplicadas al motor, eliminando los sensores mecánicos u ópticos antes citados. Además de los sensores eléctricos, la realización práctica de un observador requiere de un soporte electrónico que permita hacer evolucionar al sistema dinámico que lo representa. En estos casos, lo conveniente suele ser usar un procesador que permita no sólo esta operación, sino además, el cómputo de la estrategia de control y la elaboración de las señales de comando para la electrónica de potencia que excita al motor. Todo este cómputo debe efectuarse en tiempo real, por lo que usualmente, es necesario recurrir a un microprocesador digital de señales (DSP) de alta prestación.

Para decidir acerca del uso de sensores u observadores pueden analizarse comparativamente, los siguientes aspectos: 
- Costo: Los sensores de la resolución y precisión requeridos para un control de alto desempeño y su electrónica asociada, suelen ser considerablemente más costosos que lo que cuesta el incremento de capacidad que necesita el observador en el DSP, que ya existe para el cómputo de la estrategia de control y los comandos de excitación del motor.

- Instalación: El observador es en realidad un programa que es instalado en la placa que lleva el DSP, por lo que no requiere de una inversión extra. Los sensores de posición y velocidad deben colocarse en forma solidaria con el rotor lo que plantea típicamente el diseño de soportes especiales para su fijación, además de necesitar cableado adicional.

- Mantenimiento: Al tratarse de dispositivos mecánico/ ópticos, los sensores están sujetos al desgaste y a la acción del polvo, la humedad, etc., por lo que requieren un mantenimiento periódico. El programa de mantenimiento de placas electrónicas es usualmente, mucho más simple y está prorrateado con las otras funciones del DSP.

- Confiabilidad: Es conocido en general, que la confiabilidad de los equipos mecánicos suele ser inferior a la obtenida por equipos electrónicos.

- Interferencias EM: Los sensores producen señales eléctricas que deben transmitirse hacia el panel donde se encuentra su electrónica asociada. En ese camino, los cables que lo hacen, producen y simultánemente reciben interferencias electromagnéticas de y en otros equipos. Estas señales espurias suelen ser causas de funcionamiento errático difíciles de localizar. El observador sólo intercambia señales con los sensores de tensión y corriente del motor que son generadas, típicamente dentro del mismo panel eléctrico.

De lo anterior, es un hecho, que resulta sumamente ventajoso utilizar observadores en los accionamientos eléctricos. 
El diseño de observadores basados en los modelos de estado tiene su origen a fines de la década del 50 en los trabajos de Kalman y Bucy [53], [54] y Luenberger [71]. En un comienzo esta teoría fue aplicada en el campo del control lineal donde desarrollaron un vasto número de aplicaciones. Sin embargo, el intento por mejorar el desempeño en cierta clase de sistemas hizo que se desarrollase la teoría de control de sistemas no lineales y como lógica consecuencia la idea de diseñar observadores para este tipo de sistemas.

Los accionamientos eléctricos que emplean motores de alterna, con los que se trata actualmente, son modelados a través de ecuaciones diferenciales no lineales ya que esto permite diseñar estrategias de control que mejoran significativamente el desempeño. Por este motivo, si se pretende diseñar observadores que obtengan una adecuada estimación de la posición y la velocidad angular será necesario recurrir a métodos de diseño de observadores para sistemas no lineales.

La teoría de observadores no lineales cuenta en la actualidad con un grado de desarrollo tal que las técnicas empleadas para la construcción de los mismos, solamente pueden ser utilizadas si el sistema satisface adecuadas hipótesis. Estas hipótesis hacen que, por lo general, existan relaciones a satisfacer entre la salida (mediciones) y los estados a estimar. Una es, obviamente, la relación de observabilidad, como en el caso lineal, pero suelen aparecer otras que restringen la clase de sistemas en los cuales se pueden aplicar estos observadores. En ese sentido, el optar en los accionamientos eléctricos por el uso de observadores obliga a encarar el diseño considerando que las mediciones disponibles son las corrientes y tensiones de fase del estator. Esto fija la ecuación de salida del sistema.

\subsection{Objetivo}

En el marco del planteo de la sección anterior, se vislumbra como posible y muy provechoso el desarrollo de observadores que reemplacen a los sensores mecánicos / ópticos. El objetivo del presente trabajo es emplear las herramientas provenientes 
de la teoría del control no lineal, en pos, de desarrollar técnicas que permitan estimar la posición y la velocidad angular del rotor en accionamientos eléctricos. Dada la naturaleza no lineal de los modelos empleados para la descripción de los accionamientos, es de esperar que éstos observadores posean un mejor desempeño que aquellos construídos con técnicas que utilizan linealización. Una vez diseñado, el observador podría ser usado para la construcción de estrategias de control que no usen sensores mecánicos. Esto permitiría obtener un accionamiento más confiable y de menor costo, debido a que las estrategias de control sin sensores mecánicos podrían integrarse en un microcomputador.

\subsection{Observadores no lineales y accionamientos eléctricos}

\subsubsection{Observadores no lineales en general}

Desde principios de la década del 70 se han realizado esfuerzos para desarrollar una teoría de observadores para sistemas no lineales. Sin embargo, la mayoría de los trabajos consisten en el desarrollo de observadores para cierta clase particular de sistemas bajo la suposición de que el sistema satisface ciertas condiciones o por otro lado, observadores basados en métodos de verificación. Dentro de estos últimos podemos encontrar [59] y [114]. En estos trabajos, se proponen observadores para sistemas autónomos y empleando métodos de estabilidad de Lyapunov se garantiza la convergencia exponencial de los mismos.

Dentro de la línea que realiza observadores para sistemas que satisfacen ciertas condiciones ha tenido amplio desarrollo aquella que procura obtener observadores con dinámica del error linealizable. Los trabajos más importantes son [8], [55], [60], [61], [68], [93], [124], [125]. También, en pos de mejorar la robustez, se han desarrollado observadores de estructura variable en los trabajos [41], [105], [121], [126], [127].

Otra técnica consiste en recurrir a la pseudolinealización o linealización extendida. Esto es lo que se hace en los trabajos [5], [9], [64]. 
En [120] se presenta un estudio comparativo de estas cuatro técnicas. En [35] se realiza un exhaustivo análisis de las condiciones que debe satisfacer una clase de sistemas no lineales para que se le pueda construir un observador de tipo Luenberger, se extiende la definición de observabilidad de manera de adecuarla a este tipo de sistemas. También se realiza ahí un significativo aporte en lo que se refiere a la construcción de observadores de orden reducido [23], [37], [71] para sistemas no lineales.

\subsubsection{Aplicación en accionamientos eléctricos}

Las diferentes técnicas para la construcción de observadores se han aplicado en diversos campos. Por ejemplo, en robótica, química, y aeronáutica. En el campo específico de los accionamientos eléctricos el enfoque se centró, al principio, en los accionamientos que emplean el motor de inducción como puede verse en [63] y sus referencias, entre otros. Sin embargo, la cada vez más frecuente utilización, en los accionamientos, de motores de continua sin escobillas y de paso ha logrado que hacia fines de la década del 80 y principios de la década del 90 creciera el interés por el diseño de observadores para los mismos, como un medio para evitar el uso de sensores mecánicos.

Particularmente, para el caso de los motores de continua sin escobillas con imán permanente, se encuentra en [50] la construcción de un observador de tipo identidad que basado en linealización estima los estados del motor. Sin embargo, ningún término de corrección es propuesto para la estima de posición la cual se calcula integrando la estimación de la velocidad. Como es sabido esto produce sesgo en mayor o menor grado. En [4] y [25] se propone emplear un filtro de Kalman extendido. En este caso la velocidad de convergencia resulta difícil de precisar y no siempre se puede garantizar la convergencia del algoritmo. También se emplea linealización para estimar la posición en [76]. Un observador de estructura variable se utiliza en [34] para estimar las corrientes y luego se obtiene la posición estimada despejando de la ecuación del modelo. En [70] se propone construir un observador no lineal 
para la velocidad usando técnicas de Lyapunov y en [21] se emplea para estimar la velocidad un observador con dinámica lineal del error.

No se encuentran demasiadas propuestas para los motores de reluctancia variable que emplean sensor de posición, generalmente, conocidos como motores de reluctancia conmutada. En [73] se estiman los estados usando un observador basado en un modelo simplificado del motor de reluctancia conmutada que emplea la linealización en el método de diseño de los términos de corrección. La estimación de posición basada en una aproximación de la forma de onda de corriente que desprecia la fuerza contraelectromotriz de rotación se encuentra en [92]. Esta aproximación no es buena si la velocidad angular es significativa, pues el término despreciado es de un valor considerable. En [27] se propone un observador extendido de Luenberger de orden reducido para estimar la posición y la velocidad angular. Finalmente, la comparación de diferentes métodos para determinar la posición angular del rotor se puede hallar en [97].

\subsection{Descripción del trabajo}

El capítulo 2 tiene como objetivo plantear el conjunto de ecuaciones diferenciales no lineales que describen el comportamiento del accionamiento. Las mismas se obtienen a partir de consideraciones físicas derivadas de la teoría de la mecánica y el electromagnetismo. Hasta el presente, las técnicas empleadas para el diseño de observadores de estado en los accionamientos con motores de paso o motores de continua sin escobillas se basa, principalmente, en métodos que emplean linealización. Por este motivo, los consabidos problemas asociados a estas técnicas (localidad de la convergencia, transición entre puntos de equilibrio, etc.) se encuentran presentes en cada uno de los casos. Como excepciones al uso de alguna técnica que utiliza linealización se encuentran, [21] donde se estima la velocidad con un observador con dinámica del error linealizable; [70] donde se emplean transformaciones para estimar, únicamente, la velocidad angular y el observador de estructura variable 
propuesto en [34]. En el capítulo 3 se presentan, entre otras, las tres técnicas no lineales empleadas en los citados trabajos para la construcción de observadores no lineales y se analizan sus ventajas y desventajas para la aplicación, particularmente en cuanto a su desempeño, en la construcción de observadores que permiten estimar la posición y la velocidad angular en los accionamientos eléctricos. Las conclusiones obtenidas en este capítulo dan paso a las contribuciones de este trabajo. Las mismas son desarrolladas a partir del capítulo 4. En éste se proponen dos técnicas que permiten construir observadores no lineales de orden reducido. Las técnicas se basan en métodos no lineales y no hacen uso de la linealización. Podría decirse que la principal diferencia con lo descripto en [35], es que parte de la propuesta es utilizar la información presente en los estados que no se van a estimar, pero que son accesibles por medición. Esto es lo que se realiza en [23] y [37] para sistemas lineales. Si bien estas técnicas pueden ser empleadas en sistemas dinámicos que satisfacen ciertas condiciones, concentrados en nuestro principal objetivo usamos las mismas para estimar la posición y la velocidad angular en accionamientos que emplean el motor de reluctancia conmutada y el motor sincrónico de imán permanente. El capítulo 5 describe el comportamiento de un observador de orden reducido aplicado al motor de reluctancia conmutada. Se da una condición suficiente que permite garantizar la convergencia global del mismo. Si bien no siempre es sencillo diseñar el observador de manera de satisfacer esta condición, si esto puede hacerse en el caso bajo estudio, se logra un observador con la nada despreciable propiedad en los sistemas no lineales de convergencia global. En un ejemplo, que utiliza parámetros reales de funcionamiento se ilustra, por simulación, el comportamiento del observador propuesto. En el capítulo 6 se construye un observador de orden reducido para el motor sincrónico de imán permanente empleando la técnica de transformación presentada en el capítulo 4. Este método permite obtener un observador "casi" global que estima la posición y la velocidad angular del rotor. Presentados los resultados del capítulo 6, en el capítulo 7, incluímos el observador para el motor sincrónico de imán permanente en un esquema de control de lazo cerrado que 
utiliza a la linealización exacta por transformación y realimentación como estrategia de control. Se muestra el funcionamiento del sistema a lazo cerrado cuando las variables estimadas se emplean para construir la estrategia de control. Finalmente, en el capítulo 8 se presentan las conclusiones y se hace referencia a posibles futuros desarrollos continuando la línea de investigación del presente trabajo. 


\section{Capítulo 2}

\section{Modelo general del accionamiento eléctrico rotativo}

\section{$2.1 \quad$ Introducción}

Los dispositivos de conversión electromecánica de energía están basados en principios físicos comunes. Se aprovecha la interacción entre campos electromagnéticos para convertir energía eléctrica en energía mecánica. Sin embargo, la forma y la estructura de estos dispositivos dependen de las funciones a que se destina cada uno de ellos. En el presente trabajo nos concentraremos en los accionamientos que emplean máquinas rotativas como un medio de conversión de energía electromecánica.

Utilizaremos este capítulo para reseñar, brevemente, el modelo matemático que permite describir el funcionamiento de los accionamientos basados en máquinas rotativas. Las ecuaciones diferenciales se obtendrán a partir de consideraciones que tienen en cuenta el balance energético que se produce en el sistema. Para el tratamiento in extenso de éste y otros tópicos relacionados se puede ver [26], [32], [44]. 


\subsection{Modelo del accionamiento}

Según el principio de conservación de la energía, puede considerarse a la energía puesta en juego en la conversión electromecánica ligada de tal manera que la energía eléctrica de entrada es la suma de la energía mecánica de salida, el aumento de energía almacenada en el campo y la energía disipada. El modelo matemático que relaciona el fenómeno energético citado será descripto de la siguiente forma. En primer término describiremos el fenómeno electromagnético, considerando su interacción con el fenómeno mecánico. Luego, aplicaremos la segunda ley de Newton para describir el comportamiento mecánico.

En un convertidor electromecánico, la energía eléctrica que ingresa es en parte absorbida por el campo de acoplamiento y en parte convertida en energía mecánica. En un sistema con excitación simple, la energía del campo magnético $\left(W_{c}\right)$ desprovisto de pérdidas es función del flujo $(\lambda)$ y la posición $(\theta)$. La expresión que describe una variación incremental de la misma es:

$$
d W_{c}(\lambda, \theta)=\frac{\partial W_{c}}{\partial \lambda} d \lambda+\frac{\partial W_{c}}{\partial \theta} d \theta=i d \lambda+T_{g}^{\lambda} d \theta
$$

tal que:

$$
\begin{aligned}
i & =\frac{\partial W_{c}}{\partial \lambda} \\
T_{g}^{\lambda} & =\frac{\partial W_{c}}{\partial \theta}
\end{aligned}
$$

donde $i$ es la corriente y $T_{g}^{\lambda}$ es la cupla. La ecuación (2.3) permite obtener la expresión de la cupla generada en función de la posición y el flujo. Sin embargo, ya que nuestro propósito es valernos de la medición de la corriente de la excitación, es conviente encontrar una expresión que describa la cupla en función de la misma. Para esto podemos definir la coenergía [32], función de la corriente y la posición.

$$
W_{c}^{\prime}(i, \theta)=i \lambda-W_{c}(\lambda, \theta)
$$

Se puede calcular una variación incremental de la coenergía como:

$$
d W_{c}^{\prime}=\frac{\partial W_{c}^{\prime}}{\partial i} d i+\frac{\partial W_{c}^{\prime}}{\partial \theta} d \theta=\lambda d i+T_{g}^{i} d \theta
$$


de modo que:

$$
\begin{aligned}
\lambda & =\frac{\partial W_{c}^{\prime}}{\partial i} \\
T_{g}^{i} & =\frac{\partial W_{c}^{\prime}}{\partial \theta}
\end{aligned}
$$

Las ecuaciones (2.6) y (2.7) permiten calcular la cupla generada a partir del flujo como una función de la corriente y la posición. Para ello, se integra (2.6) a posición constante para obtener la coenergía y luego se deriva la misma respecto de la posición usando (2.7). Hasta aquí hemos considerado que hay excitación simple. Sin embargo, si el dispositivo de conversión presenta más de una excitación, se pueden considerar la coenergía que produce cada una de ellas y luego sumarlas.

En general, la forma más común de excitar las máquinas rotativas es utilizando múltiples devanados arrollados en el estator. En este caso, la ecuación que describe el circuito electromagnético que se establece es:

$$
u_{j}=\frac{d \lambda_{j}}{d t}+r i_{j}
$$

En esta expresión, $j$ representa el número de devanados que puede variar de 1 a $q$ dependiendo de la máquina. $\lambda_{j}$ es el flujo magnético que interacciona con el devanado $j, u_{j}$ es la tensión de alimentación del devanado $j, r$ la resistencia y $i_{j}$ la corriente del devanado. El flujo mágnetico $\left(\lambda_{j}\right)$ es, en general, función de la posición y de las corrientes de los devanados $\left(i_{j}, \quad j=1 \ldots q\right)$. Sin embargo, en algunas máquinas la influencia de las corrientes no propias $\left(i_{k}\right.$, con $k=1, \ldots, q$ y $\left.k \neq j\right)$ en el flujo $\left(\lambda_{j}\right)$ puede despreciarse y el mismo es sólo función de dos variables, la posición $(\theta)$ y la corriente propia $\left(i_{j}\right)$. Esta consideración equivale a despreciar las inductancias mutuas. De todas maneras, de ser necesario, la misma podría incorporarse al modelo con el sólo hecho de considerar que el flujo mágnetico $\left(\lambda_{j}\right)$ es función de la posición y de las corrientes de todos los devanados $\left(i_{j}, \quad j=1 \ldots q\right)$.

La ecuación que expresa la segunda ley de Newton permite describir el fenómeno mecánico:

$$
J \frac{d^{2} \theta}{d t^{2}}=T_{g}^{\lambda}(\lambda, \theta)-T_{c}(\theta, \omega)
$$


$T_{g}^{\lambda}$ es la cupla generada por la máquina función del flujo y la posición angular, $J$ es el momento de inercia, $T_{c}(\theta, \omega)$ representa la cupla de carga, en general función de la posición, por ejemplo un compresor, y/o la velocidad angular, por ejemplo, ventiladores y bombas de caudal. Además de la carga mecánica efectiva, en esta cupla se incluye la cupla resistente que produce el roce viscoso que es proporcional a la velocidad angular. Las ecuaciones (2.8) y (2.9) describen el modelo de la máquina. Ya que $\lambda_{j}$ es una función de $\theta$ y $i_{j}, \quad j=1 \ldots q$, la ecuación (2.8) puede ser reescrita como:

$$
\frac{d i_{j}}{d t}=\left(\frac{\partial \lambda_{j}}{\partial i_{j}}\right)^{-1}\left(-r i_{j}+u_{j}-\frac{\partial \lambda_{j}}{\partial \theta} \omega\right)
$$

donde $\omega$ es la velocidad angular. Expresando las ecuaciones (2.9) y (2.10) en forma de variables de estado, resultan las siguientes ecuaciones:

$$
\begin{aligned}
\frac{d \theta}{d t} & =\omega \\
\frac{d \omega}{d t} & =\frac{1}{J}\left(T_{g}^{i}(i, \theta)-T_{c}\right) \quad i=\left[i_{1} \ldots i_{q}\right]^{\prime} \\
\frac{d i_{j}}{d t} & =\left(\frac{\partial \lambda_{j}}{\partial i_{j}}\right)^{-1}\left(-r i_{j}+u_{j}-\frac{\partial \lambda_{j}}{\partial \theta} \omega\right) \quad j=1 \ldots q
\end{aligned}
$$

donde $T_{g}^{i}(i, \theta)$ es la cupla generada por la máquina función de la posición y las corrientes.

Este modelo, expresado en variables de estado, es el que emplearemos durante el desarrollo de este trabajo.

Análisis del modelo:

El modelo posee dos variables de estado "mecánicas", la posición angular $(\theta)$ y la velocidad angular $(\omega)$ del rotor. El resto de las variables de estado se corresponde con las corrientes de cada fase del estator de la máquina y su número es igual al número de devanados de excitación $(q)$. Por ejemplo, en un motor trifásico sincrónico con rotor de imán permanente, $q=3$ debido a que el estator es alimentado por un bobinado trifásico.

El modelo es, en general, no lineal. Esto es debido a que el flujo concatenado es una función no lineal de la posición y las corrientes. Como consecuencia de la 
relación dada en (2.7), también lo es la cupla generada.

El modelo es de la clase de los lineales analíticos. Esta clase está representada por la siguiente ecuación:

$$
\frac{d \xi}{d t}=f(\xi)+G(\xi) u
$$

En (2.14) $\xi \in R^{n}$ representa el vector de estados, $u \in R^{m}$ al vector de comando y $f(\xi)$ y $g_{j}(\xi) \quad j=1, \ldots, q$, columnas de $G(\xi)$ son campos vectoriales analíticos.

$$
\begin{aligned}
\xi & =\left[\begin{array}{lll}
\theta & \omega & \mathbf{i}
\end{array}\right]^{\prime} \\
f(\xi) & =\left[\begin{array}{lll}
\omega & \left(T_{g}^{i}(\mathbf{i}, \theta)-T_{c}\right) & \Phi
\end{array}\right]^{\prime} \\
G(\xi) & =\left[\begin{array}{ll}
g_{1} \ldots g_{q}
\end{array}\right]
\end{aligned}
$$

con

$$
\begin{aligned}
\mathbf{i} & =\left[i_{1} \ldots i_{q}\right]^{\prime} \\
\Phi & =\left[\phi_{1} \ldots \phi_{q}\right]^{\prime} \\
\phi_{j} & =\left(\frac{\partial \lambda_{j}}{\partial i_{j}}\right)^{-1}\left(-r i_{j}-\frac{\partial \lambda_{j}}{\partial \theta} \omega\right) \\
g_{j} & =\left[\begin{array}{lll}
0 & 0 & \left(\frac{\partial \lambda_{j}}{\partial i_{j}}\right)^{-1}
\end{array}\right]^{\prime} \quad j=1 \ldots q
\end{aligned}
$$

Nuestro objetivo es construir observadores que permitan estimar la posición $(\theta)$ y la velocidad angular del rotor $(\omega)$ empleando, únicamente, como variables medidas las corrientes $\left(i_{j}\right)$ y las tensiones $\left(u_{j}\right)$ de los devanados de excitación.

\subsection{Conclusión}

En este capítulo fue descripto el modelo de estado que emplearemos para representar a los accionamientos. Las ecuaciones diferenciales se obtuvieron a partir de consideraciones energéticas. En general, la ecuación que describe el flujo en función de las corrientes y la posición es diferente de acuerdo al accionamiento con que se esté tratando. 


\section{Capítulo 3}

\section{Observadores no lineales}

\subsection{Introducción}

La construcción de observadores para sistemas representados por modelos de estado lineales determinísticos invariantes en el tiempo y con control, es un tópico que al presente, se encuentra en un estado de avance tal que la mayoría de las aplicaciones responden a una teoría claramente definida. La característica principal es que la observabilidad de estos sistemas es una propiedad de tipo global. Es decir, es independiente de la región del espacio de estado donde se encuentre operando el sistema y del valor que tome la variable de comando. Por este motivo, se pueden construir observadores con convergencia exponencial de tipo global si el sistema es observable [51].

Este no es el caso, si se desea construir observadores para sistemas no lineales. Que el observador construído sea de tipo global, o sea, tenga adecuadas propiedades de convergencia en cualquier región del espacio de estado y para todo control admisible es una propiedad muy dependiente de los sistemas con que se esté tratando. El problema es que la observabilidad es una condición, generalmente, de tipo local, dependiente de un punto de operación, fijado por una señal de control y una trayectoria de estados. Además, conseguir que la convergencia sea de tipo exponencial aumenta, en general, la cantidad de hipótesis que debe satisfacer el sistema [35]. Por esta razón, las técnicas para la construcción de observadores no lineales consis- 
ten, por lo general, en diseñar observadores con ciertas propiedades suponiendo que los sistemas satisfacen hipótesis dadas, más allá de la observabilidad.

En el campo de las aplicaciones, el principal factor de mérito para el empleo de un procedimiento es considerar si el mismo, o una variante, es utilizable en el caso bajo estudio. En este capítulo presentamos, sintéticamente, los procedimientos de mayor difusión originados hasta el presente, analizando la factibilidad de aplicación en la construcción de observadores para accionamientos eléctricos.

\subsection{Observabilidad}

La posibilidad de diseñar un observador de los estados está directamente asociada a la propiedad de observabilidad que relaciona a la salida del sistema con la ecuación de estado que describe al mismo. Para el caso de un sistema lineal analítico:

$$
\begin{aligned}
\frac{d \xi}{d t} & =f(\xi)+G(\xi) u \\
y & =h(\xi)
\end{aligned}
$$

donde $\xi \in R^{n}, u \in U \subset R^{m}$ e $y \in R^{p}$. Si se considera la clase de funciones de entrada admisibles, $u: R^{+}=[0, \infty) \longrightarrow U$, esta clase es cerrada bajo la concatenación, se puede decir que [88] dos estados $x_{1}, x_{2}$ son indistinguibles (denotado $x_{1} I x_{2}$ ) si para toda función de entrada admisible $u$ la función de salida $t \rightarrow y\left(t, 0, x_{1}, u\right), t \geq 0$, del sistema para el estado inicial $x(0)=x_{1}$, y la función de salida $t \rightarrow y\left(t, 0, x_{2}, u\right), t \geq 0$ del sistema para el estado inicial $x(0)=x_{2}$, son idénticas en su dominio común de definición. El sistema se dice observable si $x_{1} I x_{2}$ implica $x_{1}=x_{2}$. Si el sistema es observable es factible construir un observador de estados a partir de la medición de la salida. Básicamente, esta definición expresa que es necesario que se pueda distinguir en la salida la evolución de los estados a medida que el tiempo transcurre. Esto debe suceder para toda función de entrada admisible y debe ser válida para todo punto de funcionamiento. En los sistemas no lineales, puede ocurrir que se puedan distinguir los estados en una región de funcionamiento, pero no en otra. Si esto sucede el sistema es localmente observable. 
Para establecer cuando el sistema es observable por definición necesitaríamos analizar todos los infinitos estados de funcionamiento posibles. Por este motivo, se ha tratado de enunciar condiciones que permitan asegurar, de manera más sencilla, cuándo un sistema es observable. Para sistemas lineales invariantes en el tiempo:

$$
\begin{aligned}
\frac{d x}{d t} & =A x+B u \\
y & =C x
\end{aligned}
$$

donde $x \in R^{n}, u \in R^{m}$ y $y \in R^{p}$, Kalman [54] demostró que la observabilidad podía analizarse fácilmente empleando un test que actualmente se denomina test de Kalman [51]. Este test establece que si la matriz:

$$
\left[\begin{array}{c}
C \\
C A \\
\vdots \\
C A^{n-1}
\end{array}\right]
$$

es de rango $n$, dimensión del sistema, el sistema es observable.

Más aún, existen otros tests que pueden ser empleados para la verificación, por ejemplo, el test de Popov-Belevitch-Hautus (PBH) [51]. Otras formas de verificar la observabilidad para sistemas lineales se pueden encontrar en [19], [51], [99].

Para sistemas no lineales de cualquier clase, los resultados obtenidos hasta el presente, no son tan generales. Sin embargo, para algunas clases particulares de sistemas e introduciendo algunas hipótesis adicionales en los campos vectoriales, ha sido posible desarrollar ciertos resultados. Por ejemplo, una condición de característiscas similares a la dada en (3.5) para sistemas lineales se ha establecido para cierta clase de sistemas no lineales. Esta puede verse en [35] donde se define cuando un sistema es observable-c. El tratamiento de aspectos de la controlabilidad, observabilidad y teoría de la realización mínima para cierta clase de sistemas no lineales se encuentra en [24], [43], [47], [88]. También pueden verse, condiciones de observabilidad, su relación con el espacio de observación y la codistribución de observabilidad en, por ejemplo, [47], [88]. 
Figura 3.1: Diagrama esquemático de un observador

\subsection{Observadores para sistemas no lineales}

Un observador de estado, es un sistema dinámico, empleado con el propósito de estimar el vector de estado, o parte del mismo. Este sistema dinámico tiene como entrada las salidas de la planta y las entradas de la misma. Una representación esquemática se encuentra en la figura 3.1. Si suponemos un modelo dinámico para la planta representado por:

$$
\begin{aligned}
\dot{x} & =f(x, u) \\
y & =h(x)
\end{aligned}
$$

El sistema dinámico observador se puede representar por:

$$
\dot{z}=w(z, u, y)
$$




$$
s=z
$$

donde $z$ es el vector de estado del observador y $s$ la ecuación de salida. Para ser lo que denominaremos un "buen" observador, la dinámica del error de estimación $(e=x-z)$ debe ser uniformemente asintóticamente estable (ver [119], por ejemplo).

Nota: La representación dada por (3.6)-(3.7) es adecuada para nuestro propósito, que es explicar brevemente, en que consiste un observador. Además no es restrictiva para nuestra aplicación, pues el modelo lineal analítico del accionamiento es de este tipo. Sin embargo, debemos aclarar que esta no es la representación más general de un sistema dinámico.

\subsubsection{Observador con dinámica lineal del error}

En esta subsección presentamos los observadores con dinámica lineal del error para sistemas no lineales del tipo lineal analítico múltiple entrada - múltiple salida. Este tipo de observador [124] es una generalización de los presentados en [8], [60], [61]. Como se recordará del Capítulo 2, el modelo de un sistema no lineal de tipo lineal analítico es:

$$
\begin{aligned}
\frac{d \xi}{d t} & =f(\xi)+G(\xi) u \\
y & =h(\xi)
\end{aligned}
$$

donde $\xi \in R^{n}$ representa el vector de estados, $u \in R^{m}$ es el vector de comando e $y \in R^{p}$ es el vector de salida. Este sistema con índices de observabilidad $l_{j}[24],[47]$, [88] y satisfaciendo

$$
\begin{aligned}
L_{g_{i}} L_{f}^{k} h_{j}\left(\xi_{0}\right) & =\left\{\begin{array}{llll}
1 & \text { si } i=j & k=l_{j}-1 \\
0 & \text { de otro } & \text { modo }
\end{array}\right. \\
\sum_{j=1}^{p} l_{j} & =n
\end{aligned}
$$

donde $g_{i}$ es la i-ésima columna de $G$ y $i=1 \ldots m, j=1 \ldots p, k=1 \ldots l_{j}-1$, se puede transformar en un entorno de $\xi_{0}$, bajo un adecuado cambio de coordenadas, 
$x=T^{-1}(\xi)$ en:

$$
\begin{aligned}
\frac{d x}{d t} & =A x+B u+K(y) \\
y & =C x
\end{aligned}
$$

con $x \in R^{n}, u \in R^{m}, y \in R^{p} \mathrm{y}$

$$
\begin{aligned}
& A=\left[\begin{array}{cccc}
A_{1} & 0 & \ldots & 0 \\
0 & A_{2} & \ldots & 0 \\
\vdots & 0 & \ddots & \vdots \\
0 & \ldots & \ldots & A_{p}
\end{array}\right] \quad(n \times n) \\
& A_{i}=\left[\begin{array}{cccc}
0 & 0 & \ldots & 0 \\
1 & 0 & \ldots & 0 \\
\vdots & \ddots & \ddots & \vdots \\
0 & \ldots & 1 & 0
\end{array}\right] \quad\left(l_{i} \times l_{i}\right) \\
& B=\left[\begin{array}{lll}
e_{1} & e_{l_{1}+1} \ldots e_{l_{1}+\ldots+l_{k_{1}+1}} \quad 0_{m-p}
\end{array}\right] \quad k=\min (m, p) \\
& C=\left[\begin{array}{ll}
e_{l_{1}}^{\prime} & e_{l_{1}+l_{2}}^{\prime} \ldots e_{l_{1}+l_{2}+\ldots+l_{p}}^{\prime}
\end{array}\right]^{\prime}
\end{aligned}
$$

con $e_{j} \in R^{n} ; j=1, \ldots, l_{1}+l_{2}+\ldots+l_{p}$ es un vector de todos ceros salvo un 1 en la posición $j$; si y sólo si se satisfacen, simultáneamente, las siguientes condiciones:

(i) La matriz

$$
\begin{array}{r}
O=\left[\begin{array}{llllll}
L_{f}^{0}\left(d h_{1}\right)^{\prime} & L_{f}^{1}\left(d h_{1}\right)^{\prime} & \ldots & L_{f}^{l_{1}-1}\left(d h_{1}\right)^{\prime} & L_{f}^{0}\left(d h_{2}\right)^{\prime} & \ldots \\
L_{f}^{l_{2}-1}\left(d h_{2}\right)^{\prime} & \ldots & L_{f}^{0}\left(d h_{p}\right)^{\prime} & \ldots & L_{f}^{l_{p}-1}\left(d h_{p}\right)^{\prime}
\end{array}\right]^{\prime}
\end{array}
$$

es no singular en $\xi_{0}$;

(ii) existe una aplicación $T$ de algún entorno abierto $V$ de $R^{n}$ en el entorno W de $\xi_{0}$ que satisface la ecuación:

$$
\begin{array}{r}
J_{T}=\frac{\partial T}{\partial x}=\left[\begin{array}{lllll}
\tau_{1} & a d_{f} \tau_{1} \ldots a d_{f}^{l_{1}-1} \tau_{1} & \tau_{2} & a d_{f} \tau_{2} \ldots a d_{f}^{l_{2}-1} \tau_{2} \ldots \\
& \tau_{p} & a d_{f} \tau_{p} \ldots a d_{f}^{l_{p}-1} \tau_{p}
\end{array}\right]
\end{array}
$$


con

$$
\begin{aligned}
& {\left[\begin{array}{ll}
\tau_{1} & \tau_{2} \ldots \tau_{p}
\end{array}\right]=\left[\begin{array}{lll}
g_{1} & g_{2} \ldots g_{p}
\end{array}\right]_{\xi=T(x)} \quad \text { si } m \geq p} \\
& {\left[\begin{array}{llll}
\tau_{1} & \tau_{2} \ldots \tau_{p}
\end{array}\right]=\left[\begin{array}{lll}
g_{1} & g_{2} \ldots g_{m} & \gamma_{1} \ldots \gamma_{p-m}
\end{array}\right]_{\xi=T(x)} \quad \text { si } m<p} \\
& {\left[\begin{array}{llll}
g_{1} & g_{2} \ldots g_{m} & \gamma_{1} \ldots \gamma_{p-m}
\end{array}\right]_{\xi=T(x)}=O^{-1} C^{\prime}}
\end{aligned}
$$

donde $\gamma_{j} j=1, \ldots, p-m$ son funciones auxiliares. Una demostración puede encontrarse en [106]. El sistema de la ecuación (3.14) es el sistema original pero expresado en nuevas coordenadas. Bajo las condiciones establecidas el sistema es observable y en las nuevas coordenadas se puede escribir en la forma canónica observador [61]. Ahora, con el sistema en la forma observador se puede construir un observador del tipo

$$
\begin{aligned}
\frac{d z}{d t} & =A z+B u+K(y)+L(y-w) \\
w & =C z
\end{aligned}
$$

y de las ecuaciones (3.14) y (3.18) se puede obtener la dinámica del error $(e=x-z)$.

$$
\frac{d e}{d t}=(A-L C) e
$$

Claramente puede verse que el error, en las nuevas coordenadas, posee dinámica lineal y que la velocidad de convergencia del observador en las nuevas coordenadas queda fijada por la adecuada elección de los autovalores de la matriz $A-L C$. La asignación de autovalores es posible ya que el par $(A, C)$ es observable. Si se desea recuperar el estado $\xi$, basta hacer $\hat{\xi}=T(z)$. Como $T$ es un difeomorfismo a medida que $e \longrightarrow 0$ se cumple que $\hat{\xi} \longrightarrow \xi$.

Análisis de aplicabilidad para la eliminación de sensores: que el modelo sea de tipo lineal analítico no es una condición restrictiva pues los modelos de los accionamientos responden a este tipo de estructura (ver Capítulo 2). Sin embargo, se encuentra un inconveniente cuando se trata que el sistema satisfaga las condiciones que están asociadas a la salida. Es posible construirle este tipo de observadores a algunos accionamientos (ver, por ejemplo, [21] y [109]) si se toma como salida la 
posición, además de las corrientes. Obviamente, para obtener esta salida es necesario un sensor de posición y hemos de recordar, que nuestro objetivo es construir observadores evitando colocar un sensor de posición.

\subsubsection{Observador de estructura variable}

El empleo de controladores de estructura variable tiene sus orígenes en los trabajos de Emelyanov (ver [28]) luego continuados por Utkin (ver [115]), entre otros. Las ideas de esta teoría han sido empleadas para el desarrollo de observadores de estructura variable [121], [120]. En el trabajo [121] se construyen observadores de estructura variable para sistemas del tipo:

$$
\begin{aligned}
\frac{d x}{d t} & =A x+f(t, x, u) \\
y & =C x
\end{aligned}
$$

donde $x \in R^{n}$ representa el vector de estado, $u \in R^{m}$ es el vector de comando e $y \in R^{p}$ es el vector de salida. Si se supone que:

$A 1$ : el par $(A, C)$ es detectable [62].

$A 2$ : existe una matriz definida positiva $Q \in R^{n \times n}$ y una función $h: R_{+} \times R^{n} \times$ $R^{m} \rightarrow R^{p}$ tal que:

$$
f(t, x, u)=P^{-1} C^{\prime} h(t, x, u)
$$

con $P$ la solución única de

$$
(A-K C)^{\prime} P+P(A-K C)=-Q
$$

si la matriz $K$ se elige de manera que $A-K C$ sea estable, lo que siempre puede hacerse si se satisface $A 1$.

$A 3$ : existe una función, $\rho(t, u)$ tal que

$$
\|h(t, x, u)\| \leq \rho(t, u)
$$

(cota uniforme en $x$ ) entonces el sistema dinámico

$$
\frac{d z}{d t}=(A-K C) z+S(z, y, \rho)+K y
$$


donde

$$
S(z, y, \rho)=\left\{\begin{array}{cc}
\frac{-P^{-1} C^{\prime} C e}{\|C e\|} \rho(t, u) & e \notin N \\
0 & e \in N
\end{array}\right.
$$

con el error $e=z-x$ y $N=\{e: C e=0\}$, es un "buen" observador para el sistema dado por la ecuación (3.21). Por lo tanto, los valores estimados $z$ convergen a $x$. La demostración se basa en establecer las condiciones que garantizan la convergencia del observador, empleando el método de Lyapunov. La dinámica del error está dada por:

$$
\frac{d e}{d t}=(A-K C) e-\frac{P^{-1} C^{\prime} C e}{\|C e\|} \rho(t, u)-P^{-1} C^{\prime} h
$$

Si se considera la función candidata de Lyapunov: $V(e)=e^{\prime} P e$, donde $P$ es la definida por la suposición $A 2$, la derivada con respecto al tiempo de esta función candidata es:

$$
\frac{d V}{d t}=-e^{\prime} Q e-2\|C e\| \rho-2 e^{\prime} C^{\prime} h
$$

Tomando la norma en el último término de (3.29) y considerando la suposición $A 3$ se obtiene:

$$
\frac{d V}{d t} \leq-e^{\prime} Q e-2\|C e\| \rho+2\|C e\| \rho<0
$$

Esta desigualdad garantiza la convergencia del observador. Además, la convergencia es exponencial, ya que:

$$
-\frac{\frac{d V}{d t}}{V} \geq \frac{e^{\prime} Q e}{e^{\prime} P e}
$$

por lo tanto:

$$
V(e(t)) \leq V\left(e_{0}, t_{0}\right) \exp ^{-\eta\left(t-t_{0}\right)}
$$

donde $\eta$ es el mínimo autovalor de $P^{-1} Q$.

Análisis de aplicabilidad para la eliminación de sensores: Como en el caso anterior, la posibilidad de construir un observador queda librada a que el sistema 
satisfaga las suposiciones $A 1-A 3$. Existe el caso de algunos accionamientos que podrían satisfacer esas condiciones. Pero eso depende de cuál sea el modelo de flujo que debe ser empleado para modelar la máquina adecuadamente (suposiciones $A 1$ y A2). Otros desarrollos de observadores de estructura variable se pueden encontrar en [41], [105], [126].

\subsubsection{Observador de tipo identidad}

Este observador aparece en [59] y [114] para sistemas no lineales autónomos. Sin embargo, el procedimiento propuesto para el diseño, puede ser extendido al caso de sistemas lineales analíticos, como se explica a continuación [108].

Lema: Dado el sistema

$$
\begin{aligned}
\frac{d x}{d t} & =f(x)+G(x) u \\
y & =h(x)
\end{aligned}
$$

donde $x \in R^{n}$ representa el vector de estado, $u \in R^{m}$ es el vector de comando y $y \in R^{p}$ es el vector de salida, el sistema dinámico:

$$
\frac{d z}{d t}=f(z)+G(z) u+K[h(x)-h(z)]
$$

es un "buen" observador, si se satisface la siguiente desigualdad:

$$
M=Q\left(\frac{\partial(f(x)+G(x) u)-K h(x)}{\partial x}\right)<0
$$

para $u$ acotada y $x$ en un compacto.

\section{Demostración:}

La dinámica del error viene dada por la siguiente ecuación:

$$
\frac{d e}{d t}=f(x)-f(z)+[G(x)-G(z)] u-K[h(x)-h(z)]
$$

el miembro derecho de esta ecuación puede ser escrito como

$$
\int_{z}^{x}\left(\frac{\partial(f(\mu)+G(\mu) u)-K h(\mu)}{\partial \mu}\right) d \mu
$$


Proponemos la función candidata de Lyapunov $V(e)=\frac{1}{2} e^{\prime} Q e, \quad Q>0$. En consecuencia, $V(e)$ es positiva y $\dot{V}=e^{\prime} Q \dot{e}$, usando (3.38) se obtiene

$$
\dot{V}=e^{\prime} Q \int_{z}^{x}\left(\frac{\partial(f(\mu)+G(\mu) u)-K h(\mu)}{\partial \mu}\right) d \mu
$$

parametrizando, $\mu=s x+(1-s) z, \quad s \in[0,1]$ obtenemos

$$
\frac{d V}{d t}=\int_{0}^{1} e^{\prime}\left(Q \frac{\partial(f(\mu(s))+G(\mu(s)) u)-K h(\mu(s))}{\partial \mu}\right) e d s
$$

Tal que $\frac{d V}{d t}$ (ecuación (3.40)) es negativa si se satisface la ecuación (3.36). Por lo tanto, si se puede diseñar la matriz $K$ de modo tal que se satisface (3.36) la ecuación que representa la dinámica del error tiene un punto de equilibrio uniformemente y asintóticamente estable en 0 .

Nota: La región de convergencia del observador es, en general, dependiente de los valores que tomen las variables de estado y las señales de comando. Se puede construir un observador de tipo más general (no necesariamente identidad), pero esto no es demasiado restrictivo en nuestra aplicación.

\section{Corolario:}

La convergencia es exponencial en un entorno del compacto.

\section{Demostración:}

$$
\frac{-\frac{d V}{d t}}{V} \geq \frac{e^{\prime} M e}{e^{\prime} Q e}
$$

o equivalentemente,

$$
V(e) \leq V\left(e_{0}, t_{0}\right) e^{-\eta\left(t-t_{0}\right)}
$$

donde $\eta$ es el mínimo autovalor de $Q^{-1} M$ [59], [99].

Análisis de aplicabilidad para la eliminación de sensores: Este tipo de observador da amplia libertad a la imaginación del diseñador. El observador podría tener una estructura más general que la tipo identidad y la función candidata de Lyapunov podría ser más compleja (el método de Lyapunov establece condiciones suficientes 
para garantizar la estabilidad de los sistemas no lineales). Por eso, el diseño empleando este método está fuertemente ligado al tipo de sistema que se esté tratando. La región de convergencia que se puede garantizar depende del caso bajo estudio. Como es sabido, esta es una limitación del método de Lyapunov porque aún cuando a través de cálculos sofisticados es posible calcular una región de convergencia, esta región es fuertemente dependiente de la función de Lyapunov elegida [39].

\subsubsection{Observador empleando linealización extendida y pseudolinealización}

La propuesta en [5] y [9] es asignar los autovalores luego de linealizar la ecuación diferencial del error alrededor de cualquier punto de equilibrio fijo [98]. En efecto, dado el sistema

$$
\begin{aligned}
\frac{d x}{d t} & =f(x, u) \\
y & =h(x)
\end{aligned}
$$

con $f$ derivable y familia de puntos de equilibrio dada por $u=\epsilon$ y $x=x_{\epsilon}$. Si se construye un observador dado por:

$$
\frac{d z}{d t}=f(z, u)+g(y)-g(h(z))
$$

con $g$ derivable, la dinámica del error está representada por la ecuación:

$$
\frac{d e}{d t}=f(x, u)-f(x-e, u)-g(y)+g(h(x-e))
$$

Si $u=\epsilon$, entonces $y_{\epsilon}=h\left(x_{\epsilon}\right)$ es un punto de equilibrio. Si se expande la ecuación (3.46) alrededor de este punto de equilibrio y se desprecian los términos de orden superior se obtiene:

$$
\frac{d e}{d t}=\left.\left[\frac{\partial f(x, \epsilon)}{\partial x}-\frac{\partial g(y)}{\partial y} \frac{\partial h(x)}{\partial x}\right]\right|_{\left(x=x_{\epsilon}, y=y_{\epsilon}\right)} e=A_{l} e
$$

Bajo adecuadas restricciones se pueden asignar los autovalores de $A_{l}$ de manera tal que la dinámica del error linealizada sea convergente. 
En [64] se presenta la construcción de un observador basado en pseudolinealización y transformación de coordenadas.

Análisis de aplicabilidad para la eliminación de sensores: Este observador emplea el método de linealización de la ecuación diferencial del error por serie de Taylor. Para cada $\epsilon$ existe una trayectoria $x_{\epsilon}$, por lo tanto la matriz $A_{l}$ no es constante. Sin embargo, teniendo en cuenta esta consideración se puede intentar un diseño que garantice que el sistema linealizado, independientemente del punto de equilibrio, es estable mediante una adecuada eleccción de la función $g$. El tamaño de la zona en la cual se garantiza estabilidad alrededor de estos puntos, depende de los términos no lineales que fueron despreciados en el desarrollo en serie.

\subsubsection{Observador para sistemas con salida lineal}

En esta subsección se considera una técnica para construir un observador para un sistema dado por:

$$
\begin{aligned}
\frac{d x}{d t} & =A x+B u+\xi(x, t) \\
y & =C x
\end{aligned}
$$

donde $\xi(x, t)$ satisface la condición de Lipschitz [22], [66] en la primer variable, con constante $L$, o sea,

$$
\left\|\xi\left(x_{1}, t\right)-\xi\left(x_{2}, t\right)\right\| \leq L\left\|x_{1}-x_{2}\right\| \quad \forall t
$$

y el par $(A, C)$ es observable. Para este sistema se puede construir un observador dado por:

$$
\frac{d z}{d t}=A z+B u+\xi(z, t)+G(y-C z)
$$

En efecto, la dinámica del error $(e=x-z)$ está dada por:

$$
\frac{d e}{d t}=(A-G C) e+\xi(x, t)-\xi(z, t)
$$


La solución de la ecuación (3.52) es:

$$
e(t)=\epsilon^{(A-G C) t} e_{0}+\int_{0}^{t} \epsilon^{(A-G C)(t-\tau)}[\xi(x, t)-\xi(z, t)] d \tau
$$

por lo tanto,

$$
\|e(t)\| \leq\left\|\epsilon^{(A-G C) t} e_{0}\right\|+\int_{0}^{t}\left\|\epsilon^{(A-G C)(t-\tau)}[\xi(x, t)-\xi(z, t)]\right\| d \tau
$$

Empleando el hecho de que $\xi$ es Lipschitz, con constante $L$, y aplicando el lema de Gronwall-Bellman [66], [119] se obtiene:

$$
\|e(t)\| \leq c_{1}\left\|e_{0}\right\| \epsilon^{\left(c_{1} L-\alpha_{1}\right) t}
$$

donde $c_{1}$ y $\alpha_{1}$ quedan definidos por la relación:

$$
\left\|\epsilon^{(A-G C) t}\right\| \leq c_{1} \epsilon^{-\alpha_{1} t}
$$

De la ecuación (3.55) es claro que si $\alpha_{1}>c_{1} L$ el error converge exponencialmente a cero.

Otra condición, similar a la anterior, para la convergencia se puede obtener si se emplea el método de Lyapunov. En efecto, si se elige la función candidata $V(e)=e^{\prime} P e$, con $P>0$ entonces:

$$
\frac{d V}{d t}=e^{\prime}\left(A_{c}^{\prime} P+P A_{c}\right) e+(\Delta \xi)^{\prime} P e+e^{\prime} P \Delta \xi
$$

donde $\Delta \xi=\xi(x, t)-\xi(x-e, t)$ y $A_{c}=A-G C$. Ya que $(A, C)$ es observable $A_{c}$ puede hacerse estable, entonces la ecuación:

$$
\left(A_{c}\right)^{\prime} P+P A_{c}=-Q
$$

con $Q>0$ tiene solución $P$ única [99]. Si $q$ es el mínimo autovalor de $Q$ y $p$ es el máximo autovalor de $P$, entonces:

$$
\frac{d V}{d t} \leq-q\|e\|^{2}+2 p L\|e\|^{2}
$$

Por lo tanto, si se pueden encontrar $q$ y $p$ tal que: $-q+2 p L<0$ entonces el observador es estable. 
Análisis de aplicabilidad para la eliminación de sensores: Este observador tiene algunas limitaciones, ya que se necesita que la salida sea lineal y el par $(A, C)$ observable. Sin embargo, si la salida no es lineal en el sistema bajo estudio puede intentarse emplear una transformación para llevar el sistema a la forma dada en la ecuación (3.48). Si la salida tiene una parte lineal,

$$
y=\bar{h}(x)+C x
$$

con $\bar{h}$ Lipschitz con constante $M$ en un entorno con intersección con el de $\xi$, y si se elige construir el observador dado en (3.51) entonces

$$
\|e(t)\| \leq\left\|\epsilon^{(A-G C) t} e_{0}\right\|+\int_{0}^{t}\left\|\epsilon^{(A-G C)(t-\tau)}(L+G M)\right\|\|e\| d \tau
$$

por lo tanto,

$$
\|e(t)\| \leq c_{2}\left\|e_{0}\right\| \epsilon^{\left(c_{1}(L+G M)-\alpha_{1}\right) t}
$$

y se puede intentar elegir $G$ para que el observador converja. Nótese que $c_{1}$ y $\alpha_{1}$ también dependen de $G$.

\subsubsection{El filtro de Kalman extendido}

El filtro de Kalman es un estimador de estados pensado para sistemas que han sido modelados en forma estocástica, y cuyo objetivo es producir un estimador de los estados insesgado, de mínima varianza. En sus orígenes, este estimador fue desarrollado para sistemas lineales con perturbaciones estocásticas en la ecuación de estado y en la ecuación de salida, modeladas por ruido blanco Gaussiano y estado inicial Gaussiano, independiente del ruido [1], [36], [49]. La estructura del filtro es la de una "mímica" del sistema, a la que se le adiciona un término proporcional al error de medición. Dada la media y varianza del vector de estado en el instante inicial, el método consiste en calcular la ganancia del término adicionado de forma tal que se minimice la varianza del error de estimación. Un tiempo después, esta idea fue extendida al caso de ruido coloreado y posteriormente fue posible desarrollar variantes para casos más generales. Cuando el sistema es modelado de forma 
no lineal puede emplearse un algoritmo que recibe el nombre de filtro de Kalman extendido [1], [36], [49], aunque se pierde la condición de optimalidad del filtro al emplear el algoritmo. El algoritmo puede ser resumido de la siguiente manera:

Dado un sistema descripto por un modelo de estado no lineal

$$
\frac{d x}{d t}=f(x, u, t)+w(t)
$$

donde:

el vector inicial es $x\left(t_{0}\right)$ es modelado como un vector aleatorio Gaussiano de media $x_{0}$ y covarianza $P_{0}, u(t)$ es el vector de control determinístico y $w(t)$ es un vector de ruido blanco Gaussiano con media cero, covarianza $Q(t)$ independiente de $x\left(t_{0}\right)$; el vector de medida disponible en tiempo discreto es modelado por

$$
y\left(t_{i}\right)=h\left(x\left(t_{i}\right), t_{i}\right)+v\left(t_{i}\right)
$$

$y\left(t_{i}\right)$, es una función no lineal del vector de estado y $v\left(t_{i}\right)$ es un vector de ruido blanco Gaussiano con media cero, covarianza $R\left(t_{i}\right)$, independiente de $x\left(t_{0}\right)$ y $w(t)$; la estimación del estado $\hat{x}(t)$ se obtiene en forma recursiva de la integración numérica de las siguientes ecuaciones:

$$
\begin{aligned}
\frac{d x}{d t} & =f(x, u, t) \\
\frac{d P}{d t} & =F^{\prime} P+P F+Q \\
F & =\frac{\partial f}{\partial x}
\end{aligned}
$$

Denominamos $t_{i}^{-}$al tiempo anterior a una medida que se realiza el el instante $t_{i} \mathrm{y}$ $t_{i}^{+}$al instante posterior. La ganancia del filtro es definida como:

$$
\begin{aligned}
K\left(t_{i}\right) & =P\left(t_{i}\right) H^{\prime}\left(t_{i}\right)\left[H\left(t_{i}\right) P\left(t_{i}^{-}\right) H^{\prime}\left(t_{i}\right)+R\left(t_{i}\right)\right]^{-1} \\
H\left(t_{i}\right) & =\frac{\partial h\left(x, t_{i}\right)}{\partial x} \|_{x=x\left(t_{i}^{-}\right)}
\end{aligned}
$$

Las ecuaciones que actualizan las ecuaciones del vector de estado y la matriz de covarianza son:

$$
\begin{aligned}
x\left(t_{i}^{+}\right) & =x\left(t_{i}^{-}\right)+K\left(t_{i}\right)\left[y\left(t_{i}\right)-h\left(x\left(t_{i}^{-}\right), t_{i}\right)\right] \\
P\left(t_{i}^{+}\right) & =P\left(t_{i}^{-}\right)-K\left(t_{i}\right) H\left(t_{i}\right) P\left(t_{i}^{-}\right)
\end{aligned}
$$


donde $x\left(t_{i}^{+}\right)$es vector de estado.

Análisis del Observador y su aplicabilidad para la eliminación de sensores: El filtro de Kalman necesita, para mantener su "optimalidad", que se conozca con buena exactitud el modelo de las perturbaciones. Esto implica conocer, con buen grado de precisión, la estadística del ruido. El algoritmo arranca con el valor de la matriz de covarianza del ruido que no se conoce si no, que muy frecuentemente, es supuesta. Esto dificulta el cálculo de la velocidad de convergencia del algoritmo. Además, la versión del filtro de Kalman extendido hace uso de linealización del sistema no lineal. Por este motivo, el valor estimado es sólo una aproximación de la estimación óptima. Cuando se emplea el filtro de Kalman extendido, no existe garantía de que el valor estimado sea cercano al óptimo.

\subsection{Aplicaciones en accionamientos}

En esta sección se comentan los principales trabajos en que se diseñan observadores para accionamientos "modernos" de tipo industrial. La totalidad de ellos se basan en alguna de las técnicas de observación enumeradas en la sección anterior. Se han agrupado los trabajos según el tipo de accionamiento que se trate.

\subsubsection{Motor de inducción}

Los motores de inducción han sido usados en los últimos tiempos, para el diseño de accionamientos de velocidad variable de alta potencia. Se han empleado algunas de las técnicas de diseño de observadores para estimar la posición y la velocidad angular de este motor. En realidad, el motor de inducción puede ser bien modelado por un sistema no lineal del tipo bilineal [6], [7]. Sin embargo, muchas veces se considera, suponiendo que la velocidad varía lentamente, un modelo lineal. En la actualidad, se encuentran en la literatura varios trabajos referidos, no solamente a la estimación de la velocidad; además se trata de estimar el flujo y los parámetros del 
motor. La aplicación de un observador basado en linealización extendida está en [91]. En [48] se aplica el filtro de Kalman extendido para identificar los parámetros del motor de inducción. La estimación conjunta de estados y parámetros, empleando también el filtro de Kalman extendido, está en [3]. Un estimador de flujo que considera variaciones de un parámetro se encuentra en [118] y aplicando observadores de estructura variable en [102]; mientras que en [89] y [90] se trata con observadores de orden reducido. En [11] se estudia la implementación de un observador de flujo basado en linealización. Un interesante trabajo de revisión es [63]. En este trabajo se presentan los principales aspectos a tener en cuenta en la estimación de estados y parámetros en las máquinas eléctricas y se describen las experiencias e los autores.

\subsubsection{Motor de imán permanente}

Uno de los primeros trabajos es [50], donde se diseña un observador basado en la linealización por serie de Taylor de la dinámica del error. En [104] ese observador es empleado en un esquema de control que utiliza linealización exacta por realimentación y transformación [24], [47], [88]. En [34] se propone el siguiente método. Se estiman las corrientes empleando un observador de estructura variable y luego se utilizan las ecuaciones del modelo para obtener una estimación de la posición. En [76] se propone obtener las variables mecánicas a partir de la linealización de la ecuaciones del modelo. El filtro extendido de Kalman es usado en [4], [25]. Un observador con dinámica lineal del error se diseña en [21], pero sólo se estima la velocidad, ya que para satisfacer las condiciones necesarias se mide la posición. Esto requiere, obviamente, un sensor de posición. También se propone por inspección un observador de orden reducido para la velocidad. Un observador no lineal de tipo identidad es propuesto en [70]. Éste permite estimar la velocidad. Luego, se propone integrar esta estimación para obtener la posición estimada. Claramente, esto puede producir sesgo en la estimación de posición. 


\subsubsection{Motor de reluctancia conmutada}

El motor de reluctancia conmutada presenta un modelo altamente no lineal. Tal es el caso, que en [73] se diseñan una serie de observadores pero considerando un modelo simplificado para reducir la complejidad. Este observador es de orden completo y se seleccionan las ganancias no lineales de los términos de corrección, empleando métodos de Lyapunov, analizando la dinámica no lineal del error linealizado alrededor del cero del error de estimación. En [74], [83] se estima la posición del rotor, luego de estimar el flujo, empleando el modelo estático que relaciona la posición y la corriente con el flujo. Un observador de orden reducido de tipo Luenberger empleando linealización se encuentra en [27]. Un trabajo de revisión que considera el empleo de observadores como una alternativa para estimar la posición del rotor es [97].

\subsection{Conclusión}

En este capítulo hemos presentado las técnicas más conocidas para el diseño de observadores no lineales. El problema general que se presenta es el de garantizar la convergencia global del observador. Este problema está asociado a la alinealidad de los sistemas con que se trata. Más aún, para sistemas no lineales hasta la observabilidad es una propiedad, en general, local. Las técnicas presentadas o leves variaciones de éstas son las que se han aplicado para la construcción de observadores de posición y/o velocidad hasta el presente. Una comparación de las técnicas y sus aplicaciones permite realizar las siguientes consideraciones:

- La característica principal es que la mayoría de los observadores son de orden completo. Esto quiere decir que estiman la totalidad del vector de estado.

- El empleo de técnicas que usan la linealización la mayoría de las veces, no garantizan la convergencia mucho más allá de una zona, por lo general restringida, del punto de operación. Si se emplean técnicas de linealización extendida 
o pseudolinealización se presenta el problema de la existencia de una transición dinámica entre puntos de operación.

- Cuando se emplea el filtro extendido de Kalman no es sencillo garantizar la convergencia del algoritmo y resulta sumamente dificultoso predecir la velocidad de convergencia del mismo. Los valores iniciales de media y covarianza del estado, en general, no son conocidos.

- En [21] se construye un observador para estimar la velocidad angular, con dinámica del error linealizable, que necesita un sensor de posición.

- Para utilizar la técnica de estructura variable como ha sido planteada es necesario que el sistema satisfaga las suposiciones $A 1-A 3$. No siempre el sistema satisface estas condiciones, por ejemplo, en [34], como fue explicado el observador de estructura variable se emplea para la estimación de corrientes, pero no se usa en la estimación de las variables mecánicas. De todos modos, estas suposiciones aparecen debido a que se eligió una función de Lyapunov cuadrática.

- Las técnicas no lineales para construir observadores de orden reducido para accionamientos aparecen poco explotadas.

Analizadas estas consideraciones, la propuesta principal consiste en emplear técnicas para la construcción de observadores de orden reducido que estimen, únicamente, la posición y la velocidad. En efecto, si analizamos el modelo de los accionamientos (ver capítulo 2) vemos que el vector de estado, está compuesto por la posición, la velocidad y las corrientes. Sin embargo, debido a que las corrientes son medidas no necesitan ser estimadas. Emplear observadores de orden reducido tiene la ventaja de disminuir la complejidad del observador. Esto facilita la implementación y el análisis de convergencia.

Respecto de este punto podemos comentar que a pesar que los observadores de orden reducido han sido tratados a nivel de sistemas lineales por [23], [37], [71], [72]; 
poco se encuentra en la literatura sobre observadores de orden reducido para sistemas no lineales. En [117] se dan condiciones para la existencia de observadores de orden reducido para sistemas no lineales autónomos. En [35] se propone un método de diseño para observadores de orden reducido de cierta clase de sistemas no lineales con control. La idea de este observador de orden reducido es emplear la información presente en las ecuaciones de salida. Esto fue explotado por Luenberger [71], [72] para la construcción de observadores de orden reducido en sistemas lineales.

La idea de [23] y [37] puede ser extendida a sistemas no lineales. De este modo, en capítulos posteriores, construiremos observadores de orden reducido para una clase de sistemas no lineales. Los observadores estiman $n-l$ de variables de estado de un sistema de $n$ variables de estado usando la medición de las $l$ variables restantes o de $l$ salidas. El empleo de estos observadores es adecuado para estimar la posición y la velocidad angular en accionamientos eléctricos. Los mismos disminuyen el orden del observador de $(q+2)$ estados ( $q$ corrientes que son medidas, posición y velocidad) a solamente dos. 


\section{Capítulo 4}

\section{Observadores de orden reducido}

\subsection{Introducción}

A pesar de que existen fuertes motivaciones para construir observadores no lineales de orden reducido, como por ejemplo, la casi segura disminución de la carga computacional en la implementación, la simplificación del estudio de estabilidad en varias aplicaciones, este tópico no ha sido demasiado desarrollado hasta el presente. En [117] se estudian las condiciones que permiten establecer cuando es posible construir observadores de orden reducido para sistemas no lineales autónomos. En [35], se propone una técnica para diseñar observadores de orden reducido para una clase de sistemas no lineales con control. Se establecen las condiciones bajo las cuales a un sistema no lineal se le puede construir un observador de tipo Luenberger. Si el sistema satisface esas condiciones se le puede construir un observador para estimar el vector de estado usando la salida y la entrada. Un inconveniente es que no siempre los sistemas satisfacen las condiciones necesarias para poder diseñar este tipo de observador. La razón más importante es que las variables medidas que originan la salida del sistema con la que se construirá el observador son elegidas, casi siempre, por razones de facilidad de medición, costo de los sensores, etc. y no por las necesidades de diseño. Esto motiva, que muchas veces condiciones que puedan parecer muy simples en algunos casos no lo sean en otros. A continuación, proponemos dos métodos para diseñar observadores no lineales de orden reducido, 
que suponen la existencia de un conjunto de variables de estado que no se desean estimar, pues las mismas se conocen, ya sea por medición directa o porque se pueden obtener a partir de la salida. Aunque esta suposición es fuerte, existen aplicaciones en las cuales se da esta situación. Concretamente, estos métodos pueden usarse en el caso de los accionamientos eléctricos cuando se desea estimar la posición y la velocidad angular del rotor a partir de las mediciones de las variables eléctricas. El observador propuesto es de tipo identidad. Copia la dinámica del modelo y a ésta se le adiciona un término de corrección que es una función no lineal de las mediciones.

El tratamiento presentado en este capítulo puede considerarse, en parte, como una extensión de los métodos empleados en [23] y en [37] para sistemas lineales, en [59] para sistemas no lineales autónomos y en [128] para sistemas no lineales con salida lineal. Restringimos el desarrollo a sistemas no lineales del tipo lineal analítico.

Suponemos que el sistema viene dado por el siguiente modelo:

$$
\begin{aligned}
& \dot{x}_{1}=f_{1}\left(x_{1}, x_{2}\right)+\sum_{r=1}^{m} g_{1}^{r}\left(x_{1}, x_{2}\right) u_{r} \\
& \dot{x}_{2}=f_{2}\left(x_{1}, x_{2}\right)+\sum_{r=1}^{m} g_{2}^{r}\left(x_{1}, x_{2}\right) u_{r}
\end{aligned}
$$

con salida

$$
y=h(x)=\left[h_{1}(x), \ldots, h_{p}(x)\right]^{\prime}
$$

donde $x=\left[\begin{array}{ll}x_{1} & x_{2}\end{array}\right]^{\prime} \in R^{n}$ representa al vector de estado y $u=\left[u_{1}, \ldots, u_{m}\right]^{\prime} \in R^{m}$, representa el vector de entrada. Sin pérdida de generalidad, podemos suponer que los estados a estimar son $\operatorname{los} x_{1} \in R^{n-l}, x_{2} \in R^{l}$ es un vector con $l$ estados, que representa a los estados que no se desean estimar.

\subsection{Técnicas propuestas}

Proponemos dos técnicas para la construcción de observadores de orden reducido. La primera emplea métodos de Lyapunov para establecer las condiciones que garantizan la convergencia exponencial del observador. La segunda utiliza transformaciones 
no lineales para poder diseñar el observador en coordenadas donde sea más sencillo garantizar la convergencia.

\section{Lema 4.1:}

Suponiendo que existe $T: U \longmapsto V$, con $U$ y $V$ abiertos, $U \in R^{n-l} \times R^{p}, V \in R^{l}$ tal que $x_{2}=T\left(x_{1}, y\right)$. El sistema dinámico

$$
\dot{\hat{x}}_{1}=f_{1}\left(\hat{x}_{1}, \hat{x}_{2}\right)+\sum_{r=1}^{m} g_{1}\left(\hat{x}_{1}, \hat{x}_{2}\right) u_{r}+\varepsilon\left(y, \hat{x}_{1}, \hat{x}_{2}, \hat{y}\right)
$$

donde $\hat{x}_{2}=T\left(\hat{x}_{1}, y\right)$ e $\hat{y}=h\left(\hat{x}_{1}, \hat{x}_{2}\right)$, es un observador asintóticamente estable para (4.1) si:

(i) $\varepsilon \longrightarrow 0$ cuando $t \longrightarrow \infty$

(ii) existe una matriz $Q \in R^{n-l} \times R^{n-l}$ positiva definida y $\sigma>0$ tal que:

$$
e^{\prime} Q\left(\frac{\partial \gamma}{\partial \mu}\right) e<-\sigma e^{\prime} e
$$

donde $e=x_{1}-\hat{x}_{1} \mathrm{y}$

$$
\begin{aligned}
\gamma= & f_{1}(\mu, T(\mu, y))+\sum_{r=1}^{m} g_{1}^{r}(\mu, T(\mu, y)) u_{r}+ \\
& \varepsilon(y, \mu, T(\mu, y), h(\mu, T(\mu, y)))
\end{aligned}
$$

con $\mu=s x_{1}+(1-s) \hat{x}_{1}, \quad s \in[0,1]$.

\section{Demostración:}

Sea la función candidata de Lyapunov:

$$
V(e)=\frac{1}{2} e^{\prime} Q e>0
$$

con $Q>0 ; e=0$ punto de equilibrio de la ecuación diferencial del error si se satisface la condición (i) y además $V(0)=0$. Su derivada con respecto al tiempo $(\dot{V})$, puede escribirse como:

$$
\dot{V}=\int_{0}^{1} e^{\prime} Q \frac{\partial \gamma}{\partial \mu} e d s
$$

$\dot{V}$ es siempre definida negativa si se satisface la desigualdad (4.5).

Nota: La transformación $T$ de be satisfacer la ecuación diferencial que surge de derivar $x_{2}$ con respecto al tiempo.

Nota: la condición (i) del lema es para establecer que el punto de equilibrio de la dinámica del error es el cero. 


\subsubsection{Región y tipo de convergencia}

$Q\left(\frac{\partial \gamma}{\partial \mu}\right)$ debe ser evaluada en los puntos de operación. Para todo punto de operación que satisfaga la desigualdad (4.5), la convergencia está asegurada en un entorno del mismo si se impone continuidad $\gamma$. En todos los casos la convergencia del observador es de tipo exponencial, ya que:

$$
\dot{V} \leq-\sigma\|e\|^{2}
$$

con $\sigma>0, \mathrm{y}$

$$
\frac{1}{2} \lambda_{\min }(Q)\|e\|^{2} \leq V \leq \frac{1}{2} \lambda_{\max }(Q)\|e\|^{2}
$$

donde $\lambda_{\min }(Q)$ y $\lambda_{\max }(Q)$ son el mínimo y máximo autovalor de la matriz $Q$. Entonces

$$
\dot{V} \leq \frac{-2 \sigma}{\lambda_{\max }(Q)} V
$$

por lo tanto,

$$
V \leq V(0) e^{\alpha t}
$$

donde $\alpha=\frac{-2 \sigma}{\lambda_{\max }(Q)}<0$. En consecuencia, $\|e\|^{2} \leq \frac{V(0)}{\lambda_{\min }(Q)} e^{\alpha t}$.

\subsubsection{El término de corrección}

En la ecuación (4.4), $\varepsilon$ es el término de corrección. El mismo debe ser diseñado para garantizar la convergencia a cero del error de estimación $\left(e=x_{1}-\hat{x}_{1}\right)$. Existen diferentes posibilidades para elegir este término. En general, cuanto más complicada sea la función elegida, más complicado será el análisis de convergencia. Ya que, $\varepsilon$ debe tender a cero cuando $t \longrightarrow \infty$ una forma de elegir el término de corrección es que el mismo sea proporcional a la diferencia de dos funciones que coinciden cuando el error de estimación es nulo. Por ejemplo, algunas de las posibles elecciones son:

1. $\varepsilon_{1}=G_{1}\left[y-h\left(\hat{x}_{1}, \hat{x}_{2}\right)\right]$ 
2. $\varepsilon_{2}=G_{2}\left[\dot{x}_{2}-\left(f_{2}\left(\hat{x}_{1}, \hat{x}_{2}\right)+\sum_{r=1}^{m} g_{2}^{r}\left(\hat{x}_{1}, \hat{x}_{2}\right) u_{r}\right)\right]$

3. Una combinación de ambos $\varepsilon_{3}=\varepsilon_{1}+\varepsilon_{2}$

Las matrices $G_{1}$ y $G_{2}$, supuestas de rango completo y de dimensiones adecuadas, deben ser diseñadas para que la región de convergencia sea lo más amplia posible y el observador presente una velocidad de convergencia adecuada. Si $G_{1}$ y $G_{2}$ no son de rango completo, el análisis se torna más dificultoso.

Nota: si se elige utilizar términos de corrección que empleen derivadas de las mediciones se debe considerar que las mediciones pueden estar contaminadas con ruido. Si esto sucede no es bueno realizar una derivada numérica. Se puede pensar en algún método que permita evitar la derivación numérica como se verá más adelante.

Nota: Los términos de correccioń se deben diseñar para satisfacer (4.5).

\subsubsection{Segunda técnica. Empleando cambio de coordenadas}

A continuación describimos otra manera de construir un observador de orden reducido para el sistema de ecuaciones (4.1) y (4.2). La técnica se basa, esencialmente, en hallar un cambio de coordenadas local de manera tal que, en las nuevas coordenadas, el subsistema a observar que surgirá de la ecuación (4.1), con salida (4.3), quede representado por la forma canónica "observador" [51], [125]. Esta nueva representación hará posible, bajo ciertas hipótesis, la construcción de un observador de orden reducido de manera más simple.

\section{Lema 4.2:}

Dado el sistema de ecuaciones (4.1), (4.2), (4.3) si:

(i) existe $T: W \longmapsto V$, con $W$ y $V$ entornos abiertos, $W \in R^{p}, V \in R^{l}$ tal que $x_{2}=T(y)$

(ii) existen números enteros $k_{1}, k_{2}, \ldots, k_{p}$, todos $\geq 0$, tales que $k_{1}+k_{2}+\ldots+k_{p}=$ 
$n-l$, y tal que la matriz:

$$
\left[\begin{array}{c}
\frac{\partial h_{1}}{\partial x_{1}} \\
\cdots \\
\frac{\partial L_{f}^{k_{1}-1} h_{1}}{\partial x_{1}} \\
\cdots \\
\frac{\partial h_{p}}{\partial x_{1}} \\
\cdots \\
\frac{\partial L_{f}^{k_{p}-1} h_{p}}{\partial x_{1}}
\end{array}\right]
$$

es no singular en un punto $x^{0}=\left(x_{1}^{0}, x_{2}^{0}\right)$.

Entonces, la aplicación

$$
\left[\begin{array}{l}
z_{1} \\
z_{2}
\end{array}\right]=\left[\begin{array}{l}
\phi_{1}\left(x_{1}, x_{2}\right) \\
\phi_{2}\left(x_{1}, x_{2}\right)
\end{array}\right]
$$

con $\phi_{1}: R^{n} \longmapsto R^{n-l}$ definida por:

$$
\begin{array}{llll}
\phi_{1}^{11}=h_{1} & \phi_{1}^{21}=h_{2} & \ldots & \phi_{1}^{p 1}=h_{p} \\
\ldots & \ldots & \ldots & \ldots \\
\phi_{1}^{1 k_{1}}=L_{f}^{k_{1}-1} h_{1} & \phi_{1}^{2 k_{2}}=L_{f}^{k_{2}-1} h_{2} & \ldots & \phi_{1}^{p k_{p}}=L_{f}^{k_{p}-1} h_{p}
\end{array}
$$

y donde $\phi_{2}: R^{n} \longmapsto R^{l}$ es la proyección ortogonal sobre $R^{l}$, representa un cambio de coordenadas local alrededor de $\left(x_{1}^{0}, x_{2}^{0}\right)$. Además, luego de aplicar este cambio el sistema (4.1), (4.3) se transforma en:

$$
\begin{aligned}
\dot{z}_{1} & =A z_{1}+\gamma\left(z_{1}, x_{2}\right)+\sum_{r=1}^{m} \alpha_{r}\left(z_{1}, x_{2}\right) u_{r} \\
y & =C z_{1}
\end{aligned}
$$

con

$$
A=\left[\begin{array}{cccc}
A_{1} & 0 & \ldots & 0 \\
0 & A_{2} & \ldots & 0 \\
\vdots & 0 & \ddots & \vdots \\
0 & \ldots & \ldots & A_{p}
\end{array}\right] \quad(n-l \times n-l)
$$




$$
\begin{gathered}
A_{i}=\left[\begin{array}{ccccc}
0 & 1 & 0 & \ldots & 0 \\
0 & 0 & 1 & \ldots & 0 \\
\vdots & \ddots & \ddots & \ddots & \vdots \\
\vdots & \ddots & \ddots & \ddots & 1 \\
0 & \ldots & \ldots & \ldots & 0
\end{array}\right] \quad\left(k_{i} \times k_{i}\right) \\
C=\left[\begin{array}{c}
e_{1} \\
\vdots \\
e_{k_{1}+1} \\
\vdots \\
e_{k_{1}+k_{2}+\ldots+1}
\end{array}\right]
\end{gathered}
$$

donde $e_{j} \in R^{n}$ es un vector fila con todos 0 , salvo un 1 en la posición $j$. Por lo tanto, el par $(A, C)$ es observable.

\section{Demostración:}

La aplicación $z=\phi(x)$ representa un cambio de coordenadas local alrededor de $x^{0}$. En efecto, las derivadas son continuas y su jacobiano es:

$$
\frac{\partial \phi(x)}{\partial x}=\left[\begin{array}{cc}
\frac{\partial \phi_{1}}{\partial x_{1}} & \frac{\partial \phi_{1}}{\partial x_{2}} \\
0 & I_{l}
\end{array}\right]
$$

que tiene rango $n$ en $\left(x_{1}^{0}, x_{2}^{0}\right)$ si y sólo si $\frac{\partial \phi_{1}}{\partial x_{1}}$ tiene rango $n-l$ en $\left(x_{1}^{0}, x_{2}^{0}\right)$. Pero $\frac{\partial \phi}{\partial x_{1}}$, es la matriz de la condición (ii); por lo tanto $\phi(x)$ es una transformación no singular si y sólo si se cumple (4.13). Esto ocurre si se satisface la condición (ii).

Sean $0<i \leq p$ y $0<j<k_{i}$, entonces:

$$
\dot{z}_{1}^{i j}=\frac{\partial L_{f}^{j-1} h_{i}}{\partial x_{1}} \dot{x}_{1}+\frac{\partial L_{f}^{j-1} h_{i}}{\partial x_{2}} \dot{x}_{2}=L_{f}^{j} h_{i}+\sum_{r=1}^{m} L_{g^{r}} L_{f}^{j-1} h_{i} u_{r}
$$

evaluando la expresión anterior en las nuevas coordenadas podemos afirmar:

$$
\dot{z}_{1}^{i j}=z_{1}^{i j+1}+\sum_{r=1}^{m} \alpha_{r}^{i j}\left(z_{1}, x_{2}\right) u_{r}
$$


donde

$$
\alpha_{r}^{i j}=L_{g^{r}} L_{f}^{j-1} h_{i}
$$

con el término de la derecha de la igualdad anterior evaluado en $\left(x_{1}, x_{2}\right)=$ $\phi^{-1}\left(z_{1}, z_{2}\right)$.

En cambio, para $j=k_{i}$ :

$$
\dot{z}_{1}^{i k_{i}}=L_{f}^{k_{i}} h_{i}+\sum_{r=1}^{m} L_{g^{r}} L_{f}^{k_{i}-1} h_{i} u_{r}
$$

y en las nuevas coordenadas:

$$
\dot{z}_{1}^{i k_{i}}=\gamma^{i}\left(z_{1}, x_{2}\right)+\sum_{r=1}^{m} \alpha_{r}^{i k_{i}}\left(z_{1}, x_{2}\right) u_{r}
$$

donde $\gamma^{i}$ es $L_{f}^{k_{i}} h_{i}$, evaluado en $\left(x_{1}, x_{2}\right)=\phi^{-1}\left(z_{1}, z_{2}\right)$. Matricialmente se tiene:

$$
\begin{aligned}
\dot{z}_{1} & =A z_{1}+\gamma\left(z_{1}, x_{2}\right)+\sum_{r=1}^{m} \alpha_{r}\left(z_{1}, x_{2}\right) u_{r} \\
y & =C z_{1}
\end{aligned}
$$

con $A$ y $C$ dadas por (4.17)-(4.19), respectivamente. donde el par $(A, C)$ está en la forma canónica "observador" del tipo Brunovsky [124].

Si las funciones $\gamma$ y $\alpha_{r}(1 \leq r \leq m)$ en (4.15) son Lipschitz [22], [66] en $z_{1}$ uniformemente con respecto a $x_{2} ; \mathrm{y}$ si los controles $u_{r}$ son acotados, entonces se satisfacen las siguientes relaciones:

$$
\begin{aligned}
& \left\|\sum \Delta \alpha_{r} u_{r}\right\| \leq \Omega\|e\| \\
& \|\Delta \gamma\| \leq \Gamma\|e\|
\end{aligned}
$$

con $e=z_{1}-\hat{z}_{1}, \Delta \gamma=\gamma\left(z_{1}, x_{2}\right)-\gamma\left(\hat{z}_{1}, x_{2}\right)$ y $\Delta \alpha_{r}=\left[\alpha_{r}\left(z_{1}, x_{2}\right)-\alpha_{r}\left(\hat{z}_{1}, x_{2}\right)\right]$.

Nota: Las constantes $\Omega$ y $\Gamma$ se denominan constantes de Lipschitz. La existencia de las mismas está garantizada si las funciones son derivables y su dominio es un compacto. 
Para el sistema dinámico expresado en la forma (4.20)-(4.21) proponemos el observador, con estados $\hat{z}_{1} \in R^{n-l}$, dado por:

$$
\begin{aligned}
\dot{\hat{z}}_{1} & =A \hat{z}_{1}+\gamma\left(\hat{z}_{1}, x_{2}\right)+\sum_{r=1}^{m} \alpha_{r}\left(\hat{z}_{1}, x_{2}\right)+G(y-\hat{y}) \\
\hat{y} & =C \hat{z}_{1}
\end{aligned}
$$

\section{Lema 4.3:}

El sistema dinámico (4.24) con salida (4.25), es un observador para (4.20)-(4.21) con controles $u_{r}$ acotados que converge asintóticamente, si existe $G$ tal que $\sigma=$ $c(\Gamma+\Omega)-\rho<0$; donde $c>0$ y $\rho>0$ son las cotas de $\left\|\epsilon^{(A-G C) t}\right\| \leq c \epsilon^{-\rho t}$, y $\Omega$ у $\Gamma$ son las establecidas en (4.22) y (4.23), respectivamente.

\section{Demostración:}

La dinámica del error $\left(e=z_{1}(t)-\hat{z}_{1}(t)\right)$ resulta:

$$
\begin{array}{r}
\dot{e}=(A-G C) e+\gamma\left(z_{1}, x_{2}\right)-\gamma\left(\hat{z}_{1}, x_{2}\right)+ \\
\sum_{r=1}^{m}\left[\alpha_{r}\left(z_{1}, x_{2}\right)-\alpha_{r}\left(\hat{z}_{1}, x_{2}\right)\right] u_{r}
\end{array}
$$

La ecuación (4.26) tiene la siguiente solución:

$$
\begin{aligned}
e(t)= & \epsilon^{(A-G C) t} e(0)+\int_{0}^{t} \epsilon^{(A-G C)(t-\tau)}\left\{\gamma\left(z_{1}, x_{2}\right)-\gamma\left(\hat{z}_{1}, x_{2}\right)+\right. \\
& \left.\sum_{r=1}^{m}\left[\alpha_{r}\left(z_{1}, x_{2}\right)-\alpha_{r}\left(\hat{z}_{1}, x_{2}\right)\right] u_{r}\right\} d \tau
\end{aligned}
$$

por lo tanto,

$$
\begin{aligned}
\|e(t)\| \leq & \left\|\epsilon^{(A-G C) t} e(0)\right\|+\int_{0}^{t} \| \epsilon^{(A-G C)(t-\tau)}\left\{\gamma\left(z_{1}, x_{2}\right)-\gamma\left(\hat{z}_{1}, x_{2}\right)+\right. \\
& \left.\sum_{r=1}^{m}\left[\alpha_{r}\left(z_{1}, x_{2}\right)-\alpha_{r}\left(\hat{z}_{1}, x_{2}\right)\right] u_{r}\right\} \| d \tau
\end{aligned}
$$

De (4.28), usando (4.22) y (4.23) se obtiene:

$$
\|e(t)\| \leq c \epsilon^{-\rho t}\|e(0)\|+\int_{0}^{t} c \epsilon^{-\rho(t-\tau)}(\Omega+\Gamma)\|e\| d \tau
$$

donde $\left\|\epsilon^{(A-G C) t}\right\| \leq c \epsilon^{-\rho t}$. Si se aplica el lema de Grownwall-Bellman [119] en (4.29) se obtiene:

$$
\|e(t)\| \leq c\|e(0)\| \epsilon^{[c(\Gamma+\Omega)-\rho] t}
$$


Es claro de (4.30) que si $\sigma=c(\Gamma+\Omega)-\rho<0$, entonces la dinámica del error es asintóticamente estable.

Nota: Pueden existir casos donde no sea posible satisfacer (4.30), ya que $c$ y $\rho$ no son independientes y dependen del valor de $G$ elegido. Por lo tanto, puede que no exista un $G$ tal que se cumple (4.30).

Suponiendo las mismas hipótesis se puede calcular, otra condición de suficiencia empleando el método de Lyapunov.

\section{Lema 4.4:}

El sistema dinámico (4.24) es un observador, que converge asintóticamente, para (4.20)-(4.21) con controles $u_{r}$ acotados, si existen $q$ y $p$ tal que $\sigma=-q+2 p(\Gamma+\Omega)<$ 0 donde $\Omega$ y $\Gamma$ son las establecidas en (4.22) y (4.23), respectivamente y $q$ y $p$, son el mínimo autovalor de $Q$ y el máximo autovalor de $P$, las matrices que satisfacen la ecuación de Lyapunov:

$$
A_{c}^{\prime} P+P A_{c}=-Q
$$

con $P>0, Q>0$ y $A_{c}=A-G C$.

\section{Demostración:}

Por ser el par $(A, C)$ observable, es posible elegir la matriz constante $G$ de modo tal que la ecuación de Lyapunov (4.31) tiene única solución [19], [51], [99]. Una manera de obtener el valor de $G$, es haciendo los cálculos como se propone en el Apéndice 4.A. Si se elige la función candidata de Lyapunov, $V=e^{\prime} P e$, la derivada respecto del tiempo es:

$$
\begin{aligned}
\dot{V}= & e^{\prime}\left(A_{c}^{\prime} P+P A_{c}\right) e+(\Delta \gamma)^{\prime} P e+\left(\sum \Delta \alpha_{r} u_{r}\right)^{\prime} P e+ \\
& e^{\prime} P(\Delta \gamma)+e^{\prime} P\left(\sum \Delta \alpha_{r} u_{r}\right)
\end{aligned}
$$

Tomando en cuenta (4.22), (4.23) y (4.31), la ecuación (4.32) puede ser acotada por:

$$
\dot{V} \leq(-q+2 p(\Gamma+\Omega))\|e\|^{2}
$$

Por lo tanto, si se pueden hallar $q$ y $p$ tal que $\sigma=-q+2 p(\Gamma+\Omega)<0$ la dinámica del error es uniformemente asintóticamente estable. 
La ecuación (4.24) describe la ecuación del observador luego de aplicar la transformación. Sin embargo, es conveniente describir al observador en las coordenadas originales. La ecuación que representa al mismo es:

$$
\begin{aligned}
\dot{\hat{x}}_{1}= & f_{1}\left(\hat{x}_{1}, T(y)\right)+\sum_{r=1}^{m} g_{1}^{r}\left(\hat{x}_{1}, T(y)\right) u_{r}+ \\
& \left(\frac{\partial \phi_{1}\left(\hat{x}_{1}, x_{2}\right)}{\partial x_{1}}\right)^{-1} G(y-\hat{y})
\end{aligned}
$$

Nota: Un caso interesante de analizar es aquel en que las constantes de $\Gamma$ y $\Omega$ tienden a cero a medida que el valor estimado se acerca al valor verdadero. En este caso siempre es posible encontrar $\sigma<0$ y el observador converge [22], [66].

\subsection{Conclusión}

En esta sección hemos presentado un par de técnicas que permiten construir observadores de orden reducido a una cierta clase de sistemas no lineales. Se establecieron condiciones suficientes para que un observador de tipo identidad pueda estimar una parte del vector de estado. El tamaño de la región de convergencia depende del caso bajo estudio y el diseño del término de corrección $(\varepsilon)$ con una de las técnicas y la "globalidad" de la transformación con la otra. El diseño de observadores de orden reducido se justifica ya que, a pesar de que existen en la actualidad un importante número de dispositivos electrónicos con gran capacidad de cómputo, es siempre de mucha utilidad reducir la cantidad de operaciones necesarias para implementar el observador. Esto reduce costo de los dispositivos de cómputo requeridos y además el de los dispositivos de adquisición (conversión $\mathrm{A} / \mathrm{D}$, etc.) que pueden ser de un valor no despreciable.

\section{Apéndice 4.A}

La notación empleada en este apéndice se puede encontrar en [15] y [42] y es la siguiente: 
$\otimes$ : producto de Kronecker.

$v e c$ : operador que apila las columnas de una matriz para formar un vector.

$e_{k}^{p}$ es un vector de dimensión $p$ con un 1 en la posición $k$ - esima y cero en otro lado.

$$
\begin{aligned}
& E_{i k}^{(n \times p)}=e_{i}^{n}\left(e_{k}^{p}\right)^{\prime} \\
& U_{n \times p}=\sum_{i}^{n} \sum_{k}^{p} E_{i k}^{(n \times p)} \otimes E_{k i}^{(p \times n)} \text { es una matriz cuadrada de dimensión }(n p \times n p)
\end{aligned}
$$
que tiene un único 1 en cada fila y cada columna. Las operaciones básicas que utilizaremos, para $A^{p \times q}, B^{q \times s}$ y $C^{s \times w}$ son:

$$
\begin{aligned}
& \operatorname{vec}(A B C)=\left(C^{\prime} \otimes A\right) \operatorname{vec}(B) \\
& \operatorname{vec}\left(A^{\prime}\right)=U_{p \times q} \operatorname{vec}(A)
\end{aligned}
$$

Operando, sobre

$$
(A-G C)^{\prime} P+P(A-G C)=-Q
$$

se obtiene:

$$
-\operatorname{vec}(Q)=\operatorname{vec}\left(A^{\prime} P\right)-\operatorname{vec}\left(C^{\prime} G^{\prime} P\right)+\operatorname{vec}(P A)-\operatorname{vec}(P G C)
$$

Por lo tanto, usando (4.35) y (4.36) se obtiene

$$
\begin{aligned}
-\operatorname{vec}(Q)= & \left(I_{n} \otimes A^{\prime}\right) \operatorname{vec}(P)+\left(A^{\prime} \otimes I_{n}\right) \operatorname{vec}(P) \\
& -\left(P \otimes C^{\prime}\right) \operatorname{vec}\left(G^{\prime}\right)-\left(C^{\prime} \otimes P\right) \operatorname{vec}(G) \\
-\operatorname{vec}(Q)= & {\left[\left(I_{n} \otimes A^{\prime}\right)+\left(A^{\prime} \otimes I_{n}\right)\right] \operatorname{vec}(P) } \\
& -\left[\left(P \otimes C^{\prime}\right) U_{n \times p}+\left(C^{\prime} \otimes P\right)\right] \operatorname{vec}(G)
\end{aligned}
$$

Entonces, reordenando

$$
\begin{array}{r}
{\left[\left(P \otimes C^{\prime}\right) U_{n \times p}+\left(C^{\prime} \otimes P\right)\right] \operatorname{vec}(G)=} \\
\operatorname{vec}(Q)+\left(I_{n} \otimes A^{\prime}\right) \operatorname{vec}(P)+\left(A^{\prime} \otimes I_{n}\right) \operatorname{vec}(P)
\end{array}
$$

Es decir, dado $A$ y eligiendo $Q$ y $P$ positiva definida, queda un sistema lineal (rectangular) del que puede despejarse $\operatorname{vec}(G)$, si existe una solución al menos. 


\section{Capítulo 5}

\section{Aplicación al MRC}

\subsection{Introducción}

Los motores de paso [56], [79], [82], [85] son empleados, entre otras aplicaciones, en periféricos de computadoras, en la industria textil, en la robótica. Estos motores pueden ser considerados como dispositivos electromecánicos digitales donde cada pulso eléctrico de entrada provoca que el rotor se mueva una cantidad discreta denominada paso del motor. Este tipo de motores puede dividirse principalmente en tres clases. Motores de reluctancia variable, motores de imán permanente (no confundir con el motor sincrónico de imán permanente) y motores híbridos. En muchas aplicaciones para obtener un adecuado desempeño, los accionamientos que utilizan estos motores trabajan empleando un sensor de posición que permite sincronizar la conmutación de las llaves con la posición del rotor. Al motor de reluctancia variable, cuando emplea un sensor de posición para ese fin, se lo denomina motor de reluctancia conmutada (MRC). Aplicaciones del MRC en diferentes tipos de accionamientos eléctricos se pueden encontrar, por ejemplo, en [122], donde se lo utiliza para accionar sobre un manipulador robótico, en [96] empleado en el accionamiento de las bombas de combustible en la industria aeroespacial. El desempeño para aplicaciones en sistemas de mediana potencia y su comparación con la del motor de inducción se encuentran en [81]. 


\subsection{Aplicación al MRC}

\subsubsection{El MRC}

El motor de reluctancia conmutada [10], [65], [78], [80] es miembro de la familia de los motores de paso de reluctancia variable, pero los procedimientos de diseño y las estrategias de control son modificadas para obtener un accionamiento eficiente de velocidad ajustable. El empleo del MRC en accionamientos eléctricos es cada vez más difundido, pues presenta un importante número de ventajas [78], [79], [85], [116]. Las principales son:

- La cupla es producida por la variación de reluctancia e independiente del sentido de la corriente. Por ello, los convertidores a utilizarse pueden ser unidireccionales.

- Debido a la ausencia de rotor bobinado, y a que posee un pequeño número de bobinados concentrados en el estator, la máquina es de bajo costo y simple construcción.

- La máquina es adecuada para operación a alta velocidad debido a la robusta construcción del rotor y ausencia de escobillas.

Este motor es una máquina de polos salientes tanto en el rotor como en el estator. Un corte transversal de la máquina puede ser representado, en forma esquemática, como en la figura 5.1. Para esta máquina, existen varias posibles combinaciones de número de polos del rotor y el estator. La máquina representada, ocho polos de estator y seis en el rotor, es la empleada con mayor frecuencia. Sin embargo, algunas veces, se utiliza la de seis polos en el estator y cuatro en el rotor. Las variables $\alpha_{r}$ y $\alpha_{s}$ representan los pasos polares rotórico y estatórico, respectivamente; mientras que $\beta_{r}$ y $\beta_{s}$ representan los arcos polares rotórico y estatórico, respectivamente. En la figura se muestra, por razones de claridad, el bobinado de una sola de las fases del estator. Las llaves que comandan el convertidor deben cerrarse y abrirse de 
Figura 5.1: Corte transversal del MRC 
manera que los polos del rotor tiendan a alinearse con la fase activa del estator. Para garantizar la rotación continua en un mismo sentido del rotor, la fase del estator debe encenderse cuando el polo rotórico y el polo estatórico comienzan a enfrentarse y apagarse cuando el polo rotórico y el polo estatórico dejan de hacerlo [10], [65], [80], [116]. Por lo tanto, para un correcto funcionamiento del accionamiento es necesario sincronizar la posición del rotor del motor con el encendido y apagado de las llaves del convertidor. Esto hace necesario el sensado de la posición del rotor aunque no se desee hacer control de la posición. Una manera de obtener la posición es utilizar un codificador de posición, ya sea un "encoder" o un "resolver". Nosotros lo haremos empleando un observador. El modelo de estado del motor puede ser descripto por las ecuaciones del capítulo 2. Para un motor con q-fases ( 2 q polos en el estator), las ecuaciones diferenciales que modelan el comportamiento dinámico son:

$$
\begin{aligned}
u_{j} & =r x_{j}+\frac{d \psi_{j}}{d t} \quad j=1, \ldots, q \\
J \frac{d^{2} \theta}{d t^{2}} & =T_{e}-T_{l}
\end{aligned}
$$

donde $u_{j}$ es la tensión aplicada a los terminales de la fase $j$ del estator, $x_{j}$ es la corriente de la fase $j$ del estator, $\psi_{j}$ es el flujo enlazado por la fase $j$ y $r$ es la resistencia del bobinado del estator; $\theta$ es la posición angular del rotor, $T_{e}$ es el momento eléctrico generado por la máquina, $T_{l}$ es el momento de carga, que puede depender de la posición $\theta$ y de la velocidad angular $\omega$, y $J$ es el momento de inercia total. La tensión de alimentación $u_{j}$ no es una variable independiente, sino que es función de la posición $\theta$, pues las fases del estator son excitadas en sincronismo con la posición del rotor.

\subsubsection{Modelo de la característica magnética}

El análisis del funcionamiento del MRC se puede realizar a partir de su característica magnética. La misma relaciona el flujo enlazado por cada fase con las corrientes en las fases y la posición del rotor. Debido a que el MRC opera, gener a lmente, bajo condiciones de alta saturación magnética, la descripción de esta carac- 
terística es muy intrincada y diversos modelos han sido propuestos para incorporar el comportamiento magnético a la descripción del MRC. Los diferentes modelos magnéticos del MRC difieren en la selección de la función $\psi_{j}$ que determina el flujo enlazado por cada fase en función de las corrientes en las fases y la posición del rotor. En general, $\psi_{j}$ es una función no lineal de $\theta$ y $x_{1}, x_{2} \ldots x_{q}$ debido a la saturación magnética. Una hipótesis comúnmente empleada en la modelización del MRC es despreciar la inductancia mutua entre las fases, de modo que $\psi_{j}=\psi_{j}\left(\theta, x_{j}\right), j=$ $1,2 \ldots q$. Es decir, que el flujo concatenado por la fase $j$ depende sólo de la corriente que alimenta el estator en esa fase $x_{j}$, y obviamente, de la posición del rotor.

Las curvas de la figura 5.2 representan la característica para una fase en un caso típico. Como puede verse en la curva, el flujo es, no sólo función de la corriente en el bobinado, sino también de la posición angular del rotor. Por lo tanto, las curvas están parametrizadas en función de la posición angular. La cupla, función de la posición normalizada, está parametrizada en función de la corriente. La adecuada descripción mediante un modelo matemático de esta característica es un tópico, que aún hoy, se encuentra en etapa de investigación. Entre las principales aproximaciones desarrolladas hasta el presente se propone en [116] un modelo que realiza una linealización por tramos de la característica magnética. La curva puede ser dividida en tres zonas. Se considera una zona denominada desalineada (zona de mínima inductancia) donde ninguna parte de la expansión polar del rotor se encuentra alineada con la expansión polar del estator. Una zona alineada (zona de máxima inductancia) donde las expansiones polares del rotor y estator se encuentran perfectamente enfrentadas. En estas zonas se considera que la inductancia se mantiene constante y en un valor máximo o mínimo. Luego se considera una tercer zona donde una parte de la expansión polar del rotor se encuentra alineada con la expansión polar del estator y la otra no. En esta zona se considera que la inductancia varía linealmente con la posición. Otra propuesta, de la que surge el modelo que emplearemos en nuestro desarrollo, es la que se encuentra en [46], [113]. En este modelo se propone una función suave para representar al flujo. Ya que la cupla desarrollada por un 
Figura 5.2: Característica magnética del MRC 
MRC es independiente del signo de la corriente de estator las fases son energizadas con corriente unipolar. Por ese motivo, se puede considerar en el modelo el caso en que $x_{j} \geq 0$ para $j=1,2 \ldots q$. El flujo es descripto por la siguiente ecuación:

$$
\psi_{j}\left(\theta, x_{j}\right)=\psi_{s}\left(1-\epsilon^{-x_{j} f_{j}(\theta)}\right) \quad x_{j} \geq 0
$$

donde $\psi_{s}$ es el flujo de saturación. La función $f_{j}(\theta)$ es una función periódica de la posición rotórica estrictamente positiva, que describe la variación de reluctancia del circuito magnético de cada fase. Se la puede representar por una serie de Fourier dada por:

$$
\begin{array}{r}
f_{j}(\theta)=a+\sum_{n=1}^{\infty}\left(b_{n} \sin \left(n N_{r} \theta-2(j-1) \pi / q\right)\right. \\
\left.+c_{n} \cos \left(n N_{r} \theta-2(j-1) \pi / q\right)\right)
\end{array}
$$

En general, se obtiene una exactitud suficiente en el modelado empleando la siguiente aproximación para $f_{j}(\theta)$ :

$$
f_{j}(\theta)=a+b \sin \left(N_{r} \theta-2(j-1) \pi / q\right)
$$

donde $a$ y $b$, son constantes adecuadas con $a>b$.

El momento eléctrico total es la suma de los momentos producidos por cada fase:

$$
T_{e}=\sum_{j=1}^{q} T_{j}\left(\theta, x_{j}\right)
$$

De acuerdo con lo expresado en el capítulo 2, el momento eléctrico de la fase $j$ puede calcularse derivando la coenergía respecto de la posición rotórica, resultando:

$$
T_{j}\left(\theta, x_{j}\right)=\frac{\psi_{s}}{f_{j}^{2}} \frac{d f_{j}}{d \theta}\left[1-\left(1+x_{j} f_{j}\right) \epsilon^{-x_{j} f_{j}}\right] \quad x_{j} \geq 0
$$

Podemos escribir el modelo representado en la ecuaciones (5.1) y (5.2) en términos de variables de estado, para obtener:

$$
\begin{aligned}
\dot{\theta} & =\omega \\
\dot{\omega} & =\frac{1}{J}\left(\sum_{j=1}^{q} T_{j}-T_{l}\right) \\
\dot{x_{j}} & =\left(\frac{\partial \psi_{j}}{\partial x_{j}}\right)^{-1}\left(-r x_{j}+u_{j}-\left(\frac{\partial \psi_{j}}{\partial \theta}\right) \omega\right)
\end{aligned}
$$


Figura 5.3: Diferentes posiciones rotóricas

donde la posición del rotor $(\theta)$, la velocidad del rotor $(\omega)$ y las corrientes de estator $\left(x_{j}\right)$ son tomadas como variables de estado.

Nota: En el modelo de la ecuación (5.8) se observa, claramente, la naturaleza periódica de las funciones involucradas. En efecto, todas las variables de estado dependen de $\theta$ a través de $f_{j}(\theta)$, quién, según la ecuación (5.5) es periódica con período $2 \pi / N_{r}$. Como consecuencia de esta periodicidad, la posición del rotor es detectable a menos de un múltiplo entero de $2 \pi / N_{r}$. Esto se debe a que existen muchas posiciones del rotor para las cuales los polos de éste presentan un distribución geométrica similar respecto de los polos del estator. En la figura 5.3 se ejemplifica esta aseveración. Vemos, que a pesar de que el rotor ha girado en sentido antihorario dos pasos polares, el circuito magnético es el mismo. 


\subsubsection{Un observador para el MRC}

En la subsección (3.4.3) (ver pág.35) se hace referencia a diferentes propuestas para el diseño de observadores para el motor de reluctancia conmutada. En esta sección proponemos un nuevo observador, basado en la medición de las corrientes y tensiones de fase del estator. Consideramos para la construcción del observador la presencia de un modelo no lineal en el que se contempla la característica magnética del MRC, interactuando con las características electromecánicas del modelo. El observador que se construye está basado en una de las técnicas propuestas en el capítulo 4 (ver Lema, pág.41). El observador será de orden reducido.

En un primer paso, consideraremos que la cupla de carga es proporcional a la velocidad, por lo tanto $T_{l}=B \omega$.

En (5.9)-(5.12) se presenta el modelo dinámico del observador:

$$
\begin{aligned}
\dot{\hat{\theta}} & =\hat{\omega}+g_{1} \varepsilon \\
\dot{\hat{\omega}} & =\frac{1}{J}\left(\sum_{j=1}^{q} \hat{T}_{j}-\hat{T}_{l}\right)+g_{2} \varepsilon \\
\dot{\hat{x}}_{j} & =\left(\frac{\partial \hat{\psi}_{j}}{\partial x_{j}}\right)^{-1}\left(-r x_{j}+u_{j}-\left(\frac{\partial \hat{\psi}_{j}}{\partial \theta}\right) \hat{\omega}\right) \\
\varepsilon & =\sum_{j=1}^{q}\left(\dot{x}_{j}-\dot{\hat{x}}_{j}\right)
\end{aligned}
$$

donde la posición estimada del rotor es $\hat{\theta}$, la velocidad estimada del rotor es $\hat{\omega}$ y las corrientes estimadas del estator son $\hat{x}_{j}$.

Nota: la dinámica del observador es copia de la del motor más el adicional de un término de corrección proporcional a $\varepsilon$, ya que $g_{1}$ y $g_{2}$ son parámetros constantes. El término de corrección se construye con la suma del error de las derivadas por las razones que se explican en la subsección de la pág. 63.

El observador descripto como en las ecuaciones (5.9)- (5.12), presenta el inconveniente de emplear la derivada de las corrientes $\left(\dot{x}_{j}\right)$. Estas no se obtienen por medición directa ni es conveniente calcularlas a partir de derivar numéricamente las corrientes, pues ello ocasionaría estimaciones ruidosas. Por este motivo se evita el 
uso de $\dot{x}_{j}$ mediante un cambio de variable en (5.9-5.12):

$$
\begin{aligned}
& \dot{\nu_{1}}=\hat{\omega}-g_{1} \sum_{j=1}^{q} \dot{\hat{x}}_{j} \\
& \dot{\nu_{2}}=\frac{1}{J}\left(\sum_{j=1}^{q} T_{j}\left(x_{j}, \hat{\theta}\right)-B \hat{\omega}\right)-g_{2} \sum_{j=1}^{q} \dot{\hat{x}}_{j} \\
& \dot{\hat{x}_{j}}=\left(\frac{\partial \hat{\psi}_{j}}{\partial x_{j}}\right)^{-1}\left(-r x_{j}+u_{j}-\left(\frac{\partial \hat{\psi}_{j}}{\partial \theta}\right) \hat{\omega}\right)
\end{aligned}
$$

donde las nuevas variables $\nu_{1}$ y $\nu_{2}$ están relacionadas con las variables $\hat{\theta}$ y $\hat{\omega}$ por las siguientes ecuaciones:

$$
\begin{aligned}
& \hat{\theta}=\nu_{1}+g_{1} \sum_{j=1}^{q} x_{j} \\
& \hat{\omega}=\nu_{2}+g_{2} \sum_{j=1}^{q} x_{j}
\end{aligned}
$$

\section{Análisis de la convergencia}

Si definimos $e_{\theta}=\theta-\hat{\theta}$ como el error de posición y $e_{\omega}=\omega-\hat{\omega}$ como el error de velocidad, la dinámica del error queda dada por las ecuaciones (5.18) y (5.19):

$$
\begin{aligned}
\dot{e_{\theta}} & =e_{\omega}-g_{1} \epsilon \\
\dot{e_{\omega}} & =\left[\frac{-B}{J} e_{\omega}+\frac{1}{J} \sum_{j=1}^{q}\left(T_{j}-\hat{T}_{j}\right)\right]-g_{2} \epsilon
\end{aligned}
$$

Esto explica la denominación de orden reducido pues, si además de la posición y velocidad se estimasen las corrientes, como en el observador de orden completo, la dinámica del error sería de orden $(q+2)$; mientras que la dinámica de (5.18)-(5.19) es de orden 2. Otra de las ventajas es que en (5.18)-(5.19) se simplifica notablemente, el análisis de estabilidad del observador. Siguiendo los lineamientos del método propuesto en el capítulo 4, se pueden calcular los valores de las ganancias $g_{1}$ y $g_{2}$. En efecto, la dinámica del error (5.18)-(5.19) es del tipo:

$$
\dot{e}=\dot{\eta}-\dot{\hat{\eta}}=\gamma(\eta, \xi)-\gamma(\hat{\eta}, \xi)
$$

con

$$
\gamma=\gamma_{1}-G \gamma_{2}
$$




$$
\begin{aligned}
\gamma_{1} & =\left[\begin{array}{c}
\omega \\
\left.\frac{-B}{J} \omega+\frac{1}{J} \sum_{j=1}^{q} T_{j}\left(x_{j}, \theta\right)\right)
\end{array}\right] \\
\gamma_{2} & =\sum_{j=1}^{q} \dot{x}_{j}
\end{aligned}
$$

donde:

$$
\begin{aligned}
\eta & =\left[\begin{array}{ll}
\theta & \omega
\end{array}\right]^{\prime} \\
\xi & =\left[\begin{array}{ll}
x_{1} \ldots x_{q} & u_{1} \ldots u_{q}
\end{array}\right]^{\prime} \\
G & =\left[\begin{array}{ll}
g_{1} & g_{2}
\end{array}\right]^{\prime}
\end{aligned}
$$

Los puntos de equilibrio de la dinámica del error son:

$$
\left(e_{\theta}, e_{\omega}\right)=(k(2 \pi / N r), 0) \quad k=0, \pm 1, \ldots
$$

Nota: Vemos que no existe un único punto de equilibrio. Sin embargo, un rápido análisis nos permite constatar que la convergencia del observador a un punto de equilibrio con error de posición no nulo, en realidad, no es otra cosa que una consecuencia de la indetectabilidad de los polos rotóricos (ver nota en la pág.58). Esto no representa ningún inconveniente desde el punto de vista del funcionamiento, pues si la posición estimada está desplazada de la verdadera un número entero de $2 \pi / N r$ nada cambia desde el punto de vista del accionamiento pues los circuitos magnéticos son los mismos.

Para poder garantizar la convergencia se debe cumplir (4.5), (ver pág.41) ó equivalentemente:

$$
e^{\prime} M e=e^{\prime}\left[\frac{M+M^{\prime}}{2}\right] e \leq-\sigma e^{\prime} e
$$

donde $M=\left(Q \frac{\partial \gamma}{\partial \mu}\right)$ y $\sigma>0$. Dada $Q$, los valores de $g_{1}$ y $g_{2}$ deben ser asignados para que se satisfaga la ecuación (5.28). Por lo tanto, es condición suficiente para garantizar la estabilidad del observador que los dos autovalores de la parte simétrica de $M$ presenten parte real negativa para todo valor de $\mu_{1}$ y $\mu_{2}$. En consecuencia, ya 
que

$$
M=Q \frac{\partial \gamma}{\partial \mu}=\left[\begin{array}{ll}
m_{11} & m_{12} \\
m_{21} & m_{22}
\end{array}\right]
$$

con

$$
Q=\left[\begin{array}{ll}
q_{1} & q_{2} \\
q_{2} & q_{3}
\end{array}\right]
$$

y

$$
\frac{\partial \gamma}{\partial \mu}=\left[\begin{array}{cc}
-g_{1} \frac{\partial \sum_{1}^{q} \dot{x_{j}}}{\partial \mu_{1}} & 1-g_{1} \frac{\partial \sum_{1}^{q} \dot{x_{j}}}{\partial \mu_{2}} \\
\frac{1}{J} \frac{\partial \sum_{1}^{q} T_{j}}{\partial \mu_{1}}-g_{2} \frac{\partial \sum_{1}^{q} \dot{x_{j}}}{\partial \mu_{1}} & \frac{-B}{J}-g_{2} \frac{\partial \sum_{1}^{q} \dot{x_{j}}}{\partial \mu_{2}}
\end{array}\right]
$$

los valores de $g_{1}$ y $g_{2}$ deben ser elegidos para que los autovalores de la parte simétrica de $M$ tengan parte real negativa. Es decir:

$$
\begin{aligned}
\operatorname{tr}\left(\frac{M+M^{\prime}}{2}\right) & =m_{11}+m_{22}<0 \\
\operatorname{det}\left(\frac{M+M^{\prime}}{2}\right) & =m_{11} m_{22}-\frac{1}{4}\left(m_{12}+m_{21}\right)^{2}>0
\end{aligned}
$$

Estas condiciones aseguran estabilidad asintótica del observador. No debe perderse de vista que la matriz $M$ que aparece en (5.32) y (5.33) es función de los puntos de funcionamiento. En consecuencia, para obtener un observador con convergencia global es necesario garantizar que estas ecuaciones se satisfacen independientemente del punto de funcionamiento.

El control del MRC requiere que las fases del estator sean alimentadas por una tensión $u_{j}$ dependiente de $\theta$. Mediante el observador, que elimina la necesidad del sensor de posición, es posible alimentar las fases con una ley $u_{j}(\hat{\theta})$. La misma demostración anterior puede ser empleada si se alimentan las fases con una ley $u_{j}(\hat{\theta})$ para obtener estabilidad asintótica de los puntos de equilibrio.

Basados en este resultado se puede intentar construir el observador para la posición y la velocidad angular, aun cuando las fases del estator sean comandadas por la posición estimada. A pesar de que el problema se redujo a la asignación de 
2 autovalores, puede que no resulte sencillo garantizar la convergencia global. Es mucho más probable encontrar un resultado de estabilidad asintótica si se acota la región de funcionamiento, en este caso, el resultado obtenido es de naturaleza local. El método propuesto permite obtener condiciones de suficiencia, por ello el tamaño de la zona de convergencia es el mínimo garantizable, pero puede suceder que para los valores de $G$ calculados la zona de convergencia sea mayor que la esperada.

\subsubsection{Algunas consideraciones}

\section{Observabilidad y saturación de las corrientes}

La forma de onda de corriente de fase típica para un MRC puede verse en la figura 5.4. La tensión de excitación es $V_{\max }$ para $t$ mayor que el tiempo de encendido y menor que el tiempo de apagado de la fase. $-V_{\max }$ para $t$ mayor que el tiempo de apagado y menor que el tiempo en que se anula la corriente y cero para el tiempo en que la corriente es nula como puede verse en la figura 5.5. Durante el tiempo que la corriente es nula no es posible observar la posición y la velocidad desde la medición de la misma pues, obviamente, si la salida es nula el sistema no es observable. Por lo tanto, la sola medición de una de las corrientes no haría posible, en principio, la construcción de un observador. Por este motivo, el término de corrección $(\varepsilon)$ fue construído usando la suma de las derivadas de las corrientes, garantizando la observabilidad.

El modo de funcionamiento del MRC es tal que cuando se corta la excitación de una fase se enciende la excitación de la fase contigua. En la figura 5.6 están representadas las cuatro corrientes de fase. Se puede ver que siempre hay una fase conduciendo y que las mismas se encienden y se apagan secuencialmente.

Nota: La mayoría de las veces, es complicado en un sistema funcionando poder estimar la velocidad y la posición en el arranque del motor. Esto se debe a que generalmente, durante el mismo se produce la saturación del actuador. Cuando se alcanza la condición de saturación las corrientes se mantienen constantes. Al no producirse variaciones de corriente no se satisface la condición de observabilidad y 
Figura 5.4: Forma de onda de corriente 
Figura 5.5: Forma de onda de tensión 
Figura 5.6: Formas de onda de corrientes 
por lo tanto no se puede garantizar la adecuada estimación de la posición y de la velocidad. Este incoveniente será ilustrado en el ejemplo más adelante.

\section{El empleo del PWM}

En casi todos los casos, los accionamientos que emplean un MRC utilizan un convertidor que funciona en base a técnicas de modulación de ancho de impulso (PWM) [45]. Este convertidor hace que se apliquen pulsos de tensión sobre las bobinas de las fases del estator, que son una carga de tipo inductivo. Las formas de onda de corriente reales, difieren de las ideales. En general, para obtener un buen aprovechamiento del sistema, los ángulos de encendido y apagado de las fases son tales que las corrientes presentan un grado de superposición. Mientras que la corriente de una de las fases aún no se extinguió, la otra fase comienza a conducir [116].

\section{Presencia de una cupla de carga desconocida}

Frecuentemente un MRC es empleado para accionar sobre una cupla de carga desconocida, por ejemplo, la cupla resistente que presenta un compresor $\left(T_{M} \cos (F \theta+\right.$ $\psi)$ ), donde $T_{M}$ es la cupla máxima y $F$ es un factor de reducción). En este caso, el modelado de la cupla de carga (proporcional a la velocidad, $B \omega$ ), no es adecuado. Una forma de mejorar el desempeño del observador, en esa situación, es tratar de estimar la cupla de carga desconocida, formulando para la misma un modelo aproximado. A menudo una buena suposición es suponer $T_{l}=T_{m}+B \omega$, con $\dot{T}_{m}=0$. Esto supone que la una cupla de carga es tan lenta que puede ser modelada como constante frente a la dinámica eléctrica del motor [17]. La segunda ecuación del MRC en (5.8) puede ser reescrita para considerar una cupla de carga desconocida, además de la cupla de fricción, como:

$$
\dot{\omega}=\frac{1}{J}\left(\sum_{j=1}^{q} T_{j}\left(\theta, x_{j}\right)-B \omega-T_{m}\right)
$$

Si se supone que $T_{m}$ es constante, entonces una nueva variable de estado para la parte desconocida de la cupla de carga es:

$$
\dot{T}_{m}=0
$$


El observador de orden reducido puede ser modificado adecuadamente, reemplazando la segunda ecuación en (5.14) por:

$$
\dot{\nu}_{2}=\frac{1}{J}\left(\sum_{j=1}^{q} T_{j}\left(\hat{\theta}, x_{j}\right)-B \hat{\omega}-\hat{T}_{m}\right)-g_{2} \sum_{j=1}^{q} \dot{\hat{x}}_{j}
$$

y adicionando una tercer variable $\nu_{3}$,

$$
\dot{\nu}_{3}=-g_{3} \sum_{j=1}^{q} \dot{\hat{x}}_{j}
$$

relacionada a $\hat{T}_{m}$ por

$$
\hat{T}_{m}=\nu_{3}+g_{3} \sum_{j=1}^{q} x_{j}
$$

Este procedimiento de extensión del vector de estado incrementa a 3 el orden del observador lo que aumenta la carga computacional en su implementación. Éste puede considerarse como un primer paso hacia la construcción de un observador adaptivo [77], [110] que está capacitado para enfrentar las variaciones de los parámetros del motor y la de ciertas condiciones de carga. El análisis realizado para la estabilidad es válido, con cambios obvios en las dimensiones, para el modelo de 3 estados y una adecuada elección de las constantes $g_{1}, g_{2}$ y $g_{3}$ garantiza la convergencia a cero del error de estimación.

\section{La inductancia mutua}

El modelo utilizado para representar el MRC, no tiene en cuenta la inductancia mutua. De ser necesario, la misma puede ser incluída en el modelo. Esto no afecta el método, ya que la condición dada por (5.32) y (5.33) no se modifica. Sin embargo, al calcular la matriz $M$, deben considerarse los términos que aparecen debido al cambio en el modelo. Pero debido al inherente modo de funcionamiento del MRC, no siempre todas las corrientes están activas al mismo tiempo. Por lo tanto, a la hora del diseño y la implementación alcanza con considerar las corrientes que son no nulas.

\section{Aspectos de implementación}

El empleo de modelos discretos en sistemas lineales [2], permite evitar la integración numérica para encontrar la solución y predecir la estabilidad del algoritmo de 
cómputo con un certeza absoluta, obviamente admitiendo que el modelo es exacto. Sin embargo, esto no es posible en la implementación de la resolución de ecuaciones diferenciales no lineales. En este caso es necesario recurrir a la integración mediante algún método numérico. La elección del método implica, en general, un compromiso entre sencillez, exactitud y convergencia del algoritmo. Por ejemplo, en [27], se utiliza para la resolución el algoritmo de Euler. Este es uno de los más sencillos, pero no es demasiado confiable desde el punto de vista de la convergencia. Un algoritmo muy difundido, debido a que presenta una buena solución para el citado compromiso, es el algoritmo Runge-Kutta [95]. A pesar de que no trataremos con la óptima elección de las rutinas numéricas necesarias para la implementación, realizaremos una estimación de la capacidad de cómputo necesaria suponiendo que se emplea un algoritmo del tipo Runge-Kutta de 4to. orden. La implementación del observador no lineal necesita de la integración en tiempo real de las ecuaciones diferenciales que describen al mismo. Con un observador de orden completo $q+2$ ecuaciones deberían ser integradas. Si empleamos el observador de orden reducido ese número se reduce a 2. Consideraremos un MRC que tiene 8 polos en el estator (4 fases) y 6 en el rotor.

Para calcular los valores de la derivada en un instante de tiempo es necesario realizar 31 sumas, 58 multiplicaciones y 8 divisiones. Además es necesario calcular 4 senos, 4 cosenos, 4 exponenciales y 4 señales de comando. Para calcular la salida, 10 operaciones. Además es necesario resolver dos ecuaciones diferenciales por el método de Runge-Kutta de 4to. orden.

Considerando que para el seno, el coseno y la exponencial se utilizan 10 operaciones, el total de operaciones necesarias es menor que 1000. Con los valores habituales de los motores, se puede estimar que un tiempo de muestreo de $100 \mu s e g$ es aceptable. Entonces, considerando, que un DSP realiza una operación por ciclo, son necesarias $10 \mathrm{Mflops} / \mathrm{seg}$, lo que es fácilmente alcanzado por un DSP del tipo TMS320C30, por ejemplo. 
Figura 5.7: Sistema simulado

\subsection{Resultados de simulación}

En esta sección ilustramos por simulación el comportamiento del observador propuesto. La figura 5.7 muestra el sistema simulado. Al observador ingresan las mediciones de corrientes y tensiones de fase del estator del motor y éste estima la velocidad y la posición angular del rotor. Las variables estimadas, posición y/o velocidad, son empleadas para calcular las tensiones de alimentación del estator $u_{j}$. Los parámetros y datos del MRC usado en la simulación son los dados en la siguiente tabla: 


\begin{tabular}{||c|c||}
\hline$P_{N}$ & $7.5 \mathrm{~kW}$ \\
$\Omega_{N}$ & $1900 \mathrm{r} / \mathrm{min}$ \\
$V_{N}$ & $460 \mathrm{~V}$ \\
$I_{N}$ & $32 \mathrm{~A}$ \\
$q$ & 4 \\
$N_{r}$ & 6 \\
$r$ & $0.3 \mathrm{ohm}$ \\
$\psi_{s}$ & $1 \mathrm{~Wb}$ \\
$a$ & $0.1381 / \mathrm{A}$ \\
$b$ & $0.1271 / \mathrm{A}$ \\
$B$ & $0.01 \mathrm{Nms} / \mathrm{rad}$ \\
$J$ & $0.0016 \mathrm{kgm}^{2}$ \\
\hline
\end{tabular}

La alimentación emplea un PWM con tensión máxima $460 \mathrm{~V}$ y período $10^{-4} \mathrm{seg}$. Estos valores de los parámetros hacen que el MRC utilizado en la simulación reproduzca el MRC de [16].

En las figuras 5.8-5.17 admitimos que el motor se encuentra cargado con una cupla proporcional a la velocidad angular. Siguiendo el procedimiento de diseño, por búsqueda intensiva, obtenemos:

$$
Q=\left[\begin{array}{cc}
0.5 & -1.010^{-4} \\
-1.010^{-4} & 0.2510^{-6}
\end{array}\right] \quad G=\left[\begin{array}{c}
-0.08 \\
-0.23
\end{array}\right]
$$

Estos valores de $Q$ y $G$ satisfacen (5.32) y (5.33) (ver Apéndice 5.A), y por este motivo $G$ fue utilizada como la ganancia del observador.

En todas las simulaciones el MRC alcanza el estado estacionario con una tensión de alimentación función de la posición verdadera $\left(u_{j}(\theta)\right)$. Una vez en este punto el observador es arrancado y la velocidad y la posición estimadas son empleadas para calcular las tensiones de alimentación $\left(u_{j}(\hat{\theta})\right)$.

En las figuras 5.8-5.10 se representan las trayectorias para el motor funcionando a su velocidad nominal $(\omega=198 \mathrm{rad} / \mathrm{s})$. El observador fue arrancado a partir de 
Figura 5.8: Velocidad y velocidad estimada vs. tiempo para difentes valores de posición estimada inicial 
Figura 5.9: Error de posición vs. tiempo para diferentes valores del error de posición inicial 
diferentes valores de posición inicial $\hat{\theta}(0)$ en el rango $\left(-\pi / N_{r}, \pi / N_{r}\right)=(-\pi / 6, \pi / 6)$ y con velocidad inicial cero, $\hat{\omega}(0)=0$. Cualquier error de posición inicial puede ser reducido a un rango de error equivalente $\left(-\pi / N_{r}, \pi / N_{r}\right)$. La figura 5.8 muestra la velocidad real y la estimada durante el transitorio. Se puede ver que todas las trayectorias de la velocidad se superponen para los diferentes valores de $\hat{\theta}(0)$. Este hecho demuestra que la velocidad de convergencia de la velocidad es, prácticamente, independiente del error inicial de posición. Una aseveración similar puede ser hecha si se mira el error inicial de velocidad.

La figura 5.9 ilustra la convergencia del error de posición, mientras que en la figura 5.10 se amplifica la zona alrededor del instante inicial. Se observa que la posición converge a 0 o a un múltiplo de $2 \pi / N_{r}$, dependiendo de las condiciones iniciales del observador. Esto es una consecuencia natural del modo de trabajo del motor que, como ya fue indicado, no acarrea ningún incoveniente. Como se recordará la posición no podía ser distinguida a menos de un múltiplo de $2 \pi / N_{r}$; en nuestro caso, $2 \pi / 6=1.047 \mathrm{rad}$. Durante el transitorio de convergencia el observador se "engancha", no necesariamente con el polo que da error cero. Como fue expresado este error no afecta el funcionamiento del sistema. Además, puede verse cómo luego de superado el transitorio de $0.5 \mathrm{seg}$., aproximamente, la velocidad alcanza su valor de equilibrio de estado estacionario. La convergencia de la posición es más rápida que la de velocidad pero la posición oscila alrededor del estado estacionario. Esta oscilación no se debe al PWM sino que se debe al error de velocidad. La misma desaparece cuando la estimación de la velocidad alcanza la velocidad verdadera.

En las figuras 5.11 y 5.12 ilustramos el comportamiento de la dinámica del error de posición alrededor de los puntos de equilibrio. En los gráficos se representa el error de posición $\left(e_{\theta}\right)$ en el eje de abscisa y la derivada del error de posición $\left(\dot{e}_{\theta}\right)$ en el eje de ordenadas. Como se recordará, para el error de posición los puntos de equilibrio están en $\dot{e}_{\theta}=0 \mathrm{rad} / \mathrm{seg}$ y $e_{\theta}=2 \pi / 6 \mathrm{rad}=1.047 \mathrm{rad}$. En ambas figuras puede verse como es la convergencia para diferentes valores iniciales del error de posición separados $2 \pi / 6$ rad. En el caso de la figura 5.11 las curvas se corresponden 
Figura 5.10: Error de posición (alrededor del instante inicial) para diferentes valores del error inicial, $\theta(0)=0.216 \mathrm{rad} .-\hat{\theta}(0)=-\pi / 6 \mathrm{rad},-\cdot-\hat{\theta}(0)=-\pi / 12 \mathrm{rad}$, $\cdots \hat{\theta}(0)=0 \mathrm{rad},--\hat{\theta}(0)=\pi / 12 \mathrm{rad},-\hat{\theta}(0)=\pi / 6 \mathrm{rad}$ 
Figura 5.11: Plano de fase del error de posición. - error de posición inicial -0.747 rad, -- error de posición inicial $0.3 \mathrm{rad}, \cdots$ error de posición inicial $1.347 \mathrm{rad}$ 
Figura 5.12: Plano de fase del error de posición. - error de posición inicial -1.147 rad, -- error de posición inicial $-0.1 \mathrm{rad}, \cdots$ error de posición inicial $0.947 \mathrm{rad}$ 
Figura 5.13: Velocidad y velocidad estimada vs. tiempo, para difentes valores de posición estimada inicial

con $e_{\theta}(0)=-0.747 \mathrm{rad}, e_{\theta}(0)=0.3 \mathrm{rad}$ y $e_{\theta}(0)=1.347 \mathrm{rad} ;$ mientras que en el caso de la figura $5.12 e_{\theta}(0)=-1.147 \mathrm{rad}, e_{\theta}(0)=-0.1 \mathrm{rad}$ y $e_{\theta}(0)=0.947 \mathrm{rad}$. Claramente puede distinguirse la simetría de las curvas de convergencia. Cada punto de equilibrio separado $2 \pi / 6$ funciona como un "atractor" y las trayectorias en el plano de la fase son idénticas a menos del factor de desplazamiento $2 \pi / 6$.

Las figuras 5.13-5.15 reproducen los errores de velocidad y posición para el motor funcionando a $\omega=125 \mathrm{rad} / \mathrm{seg}$. La naturaleza de las curvas es muy similar a las de las figuras 5.8-5.10. Esto muestra que la convergencia del observador es cualitativamente independiente del punto de operación, como fue especificado en el método de diseño. 
Figura 5.14: Error de posición vs. tiempo, para diferentes valores del error de posición inicial 
Figura 5.15: Error de posición (alrededor del instante inicial) para diferentes valores del error inicial, $\theta(0)=0.09 \mathrm{rad} .-\hat{\theta}(0)=-\pi / 6 \mathrm{rad},-\cdot-\hat{\theta}(0)=-\pi / 12 \mathrm{rad}$, $\cdots \hat{\theta}(0)=0 \mathrm{rad},--\hat{\theta}(0)=\pi / 12 \mathrm{rad},-\hat{\theta}(0)=\pi / 6 \mathrm{rad}$ 
Figura 5.16: Velocidad y velocidad estimada para diferentes valores de $g_{2} \cdot g_{2}=-2$, ... velocidad, - - - velocidad estimada; $g_{2}=-0.23$, - velocidad, - - velocidad estimada

Obviamente, la solución de las inecuaciones (5.32) y (5.33) no es única. El valor de ganancia $g_{2}$ fue cambiado a -2 y en la figura 5.16 y 5.17 se muestra la velocidad real y la velocidad estimada para los dos valores de la ganancia de aceleración del observador $\left(g_{2}=-0.23\right.$ y $\left.g_{2}=-2\right)$. Se ve (figura 5.16) que la velocidad de convergencia aumenta con el valor absoluto de $g_{2}$. Sin embargo, también puede verse que el incremento en la velocidad de convergencia trae aparejado un incremento en el "ripple" de la velocidad estimada. Este hecho no siempre es aceptable si se desea hacer un control de velocidad a lazo cerrado. Por lo tanto, la elección de $g_{2}$ es un compromiso. De todos modos el "ripple" ocurre solamente durante el transitorio de 
Figura 5.17: Ampliación de la figura 5.16 
convergencia.

\section{Variación de la cupla de carga}

Hasta aquí hemos presentado resultados que hacen al comportamiento del observador cuando la cupla de carga se modela como proporcional a la velocidad angular del rotor. Sin embargo, como hemos expresado, algunas veces se necesita que el MRC accione sobre una carga desconocida y en general variable. En la mayoría de los casos la carga mecánica varía lentamente comparada con la variación de la dinámica del motor y por lo tanto puede modelarse como constante. En la figura 5.18 se muestra como se comporta el observador cuando existe un desajuste en el modelo del motor empleado en el observador. Un cambio súbito de $100 \%$ en $B$, es aplicado al motor. El valor de $B$ en el modelo se mantiene sin cambios. Dado que el observador se basa en el modelo del motor, el mismo no puede estimar perfectamente la velocidad y se produce un error de estado estacionario entre la velocidad y la velocidad estimada. Por lo tanto, como era de esperar, la estimación de velocidad converge a otro valor de estado estacionario. El valor del error de estado estacionario depende de las ganancias del observador. Sin embargo, la figura muestra cómo el sistema preserva la estabilidad.

\section{Estimación de la cupla de carga}

Para componer esta situación se puede diseñar el observador extendido que estima la cupla, con valores

$$
G=\left[\begin{array}{c}
-0.08 \\
-0.23 \\
-0.03
\end{array}\right]
$$

como ya fue explicado (ver pág.67). En la figura 5.19-5.21 se ilustra el comportamiento del observador extendido que estima la cupla de carga (ecuaciones (5.36)(5.38)). Supongamos que se aplica una cupla como la que se muestra en la figura 5.19. La misma produce variaciones del orden del 5 al $10 \%$ de la cupla nominal. En la misma figura puede verse la cupla estimada. Para este caso, la figura 5.20 ilustra el error de posición estimado. Vemos el transitorio que se produce cuando se aplica 
Figura 5.18: Comportamiento ante variaciones de la cupla de carga. - - velocidad estimada, - velocidad real 
Figura 5.19: - Cupla de carga y - - cupla de carga estimada

el cambio súbito de la cupla. Sin embargo, el observador converge. Similarmente, se puede ver la convergencia de la velocidad en la figura 5.21. Obviamente, dado que el objetivo es mostrar el comportamiento del observador, estas simulaciones no incluyen un controlador de velocidad, por eso las formas de onda de velocidad lucen "ruidosas". Finalmente, se ilustran las formas de ondas de corriente (figura 5.22) cuando el observador funciona desde el arranque del motor. En la figura 5.23 puede verse el comportamiento del error de velocidad durante el transitorio de convergencia. Se observa que se produce un cambio abrupto en la convergencia cuando la corriente satura. Durante ese tiempo no se cumple la condición de observabilidad. Sin embargo, en este caso particular, el sistema se acomoda y después de un tiempo la velocidad estimada converge a la velocidad verdadera. 
Figura 5.20: Error de posición 
Figura 5.21: Velocidad y velocidad estimada 
Figura 5.22: Forma de ondas de corrientes 
Figura 5.23: Error de velocidad 


\subsection{Conclusión}

En este capítulo presentamos un nuevo método para el diseño de un observador que permite estimar la posición y la velocidad angular del rotor de un MRC. El método considera el funcionamiento del accionamiento cuando para la conmutación de las llaves se utiliza la información proveniente de la posición estimada. El tamaño de la región de convergencia depende de que se pueda encontrar valores de $G$ que permitan garantizar el cumplimiento de las desigualdades (5.32) y (5.33) en esa región. En el ejemplo de aplicación, que considera valores reales de funcionamiento, fue posible garantizar la convergencia en una amplia región que abarca los puntos de funcionamiento usuales.

El método de diseño empleado puede ser aplicado aún cuando el flujo sea modelado por una ecuación distinta a (5.3), (ver por ejemplo, [13], [16], [113]), ya que el método de diseño es independiente del modelo propuesto. Las principales ventajas que surgen del uso de un observador de orden reducido son:

- Disminución del número de operaciones para la implementación.

- Una simplificación en el método de diseño del observador.

- Un análisis más simple de su "robustez".

La primera permite disminuir la carga computacional para la implementación y la segunda permite garantizar la estabilidad analizando una matriz $(M)$ de $2 \times 2$.

La técnica puede ser extendida al caso en que exista variación de parámetros empleando un esquema adaptivo. A pesar de que puede no ser fácil encontrar una matriz $G$ que permita satisfacer las desigualdades (5.32) y (5.33) para todo punto de funcionamiento, si se logra este objetivo se habrá conseguido un observador con convergencia global.

Respecto del empleo del PWM en el control de accionamientos se debe tomar en cuenta el comentario que se realiza, en las conclusiones finales de la tesis. 


\section{Apéndice 5.A}

En este apéndice se calcula la matriz M. Con

$$
\begin{aligned}
& Q=\left[\begin{array}{ll}
q_{1} & q_{2} \\
q_{2} & q_{3}
\end{array}\right]>0 \\
& M=\left[\begin{array}{ll}
m_{11} & m_{12} \\
m_{21} & m_{22}
\end{array}\right] \\
& m_{11}=-q_{1} g_{1} \sum_{j=1}^{4} \frac{\partial \dot{x}_{j}}{\partial \mu_{1}}+\frac{q_{2}}{J} \sum_{j=1}^{4} \frac{\partial T_{j}}{\partial \mu_{1}}-q_{2} g_{2} \sum_{j=1}^{4} \frac{\partial \dot{x}_{j}}{\partial \mu_{1}} \\
& m_{12}=q_{1}-q_{1} g_{1} \sum_{j=1}^{4} \frac{\partial \dot{x}_{j}}{\partial \mu_{2}}-q_{2} \frac{B}{J}-q_{2} g_{2} \sum_{j=1}^{4} \frac{\partial \dot{x}_{j}}{\partial \mu_{2}} \\
& m_{21}=-q_{2} g_{1} \sum_{j=1}^{4} \frac{\partial \dot{x}_{j}}{\partial \mu_{1}}+\frac{q_{3}}{J} \sum_{j=1}^{4} \frac{\partial T_{j}}{\partial \mu_{1}}-q_{3} g_{2} \sum_{j=1}^{4} \frac{\partial \dot{x}_{j}}{\partial \mu_{1}} \\
& m_{22}=q_{2}-q_{2} g_{1} \sum_{j=1}^{4} \frac{\partial \dot{x}_{j}}{\partial \mu_{2}}-q_{3} \frac{B}{J}-q_{3} g_{2} \sum_{j=1}^{4} \frac{\partial \dot{x}_{j}}{\partial \mu_{2}} \\
& \frac{\partial T_{j}}{\partial \mu_{1}}=\Gamma-\Psi-\Gamma \Phi-\Delta \Lambda \\
& \Gamma=-2 \psi_{s}\left[a+b \sin \left(N_{r} \mu_{1}-\rho_{j}\right)\right]^{-3} \\
& \left(b N_{r}\right)^{2} \cos ^{2}\left(N_{r} \mu_{1}-\rho_{j}\right) \\
& \Psi=\psi_{s}\left[a+b \sin \left(N_{r} \mu_{1}-\rho_{j}\right)\right]^{-2} \\
& b N_{r}^{2} \sin \left(N_{r} \mu_{1}-\rho_{j}\right) \\
& \Phi=1-\left\{1+x_{j}\left[a+b \sin \left(N_{r} \mu_{1}-\rho_{j}\right)\right]\right\} \\
& e^{-x_{j}\left(a+b \sin \left(N_{r} \mu_{1}-\rho_{j}\right)\right.} \\
& \Lambda=e^{-x_{j}\left(a+b \sin \left(N_{r} \mu_{1}-\rho_{j}\right)\right.}\left[x_{j} b N_{r} \cos \left(N_{r} \mu_{1}-\rho_{j}\right)+x_{j}^{2} a b N_{r} \cos \left(N_{r} \mu_{1}-\rho_{j}\right)\right. \\
& \left.+x_{j}^{2} b \sin \left(N_{r} \mu_{1}-\rho_{j}\right)\right] \\
& +N_{r} \cos \left(N_{r} \mu_{1}-\rho_{j}\right) e^{-x_{j}\left(a+b \sin \left(N_{r} \mu_{1}-\rho_{j}\right)\right.}
\end{aligned}
$$




$$
\begin{aligned}
\frac{\partial \dot{x}_{j}}{\partial \mu_{1}}= & -\left[\psi_{s} e^{-x_{j}\left(a+b \sin \left(N_{r} \mu_{1}-\rho_{j}\right)\right.}\right. \\
& \left(a+b \sin \left(N_{r} \mu_{1}-\rho_{j}\right)\right]^{-2}\left(-r x_{j}+u_{j}\right) \\
& \psi_{s}\left[e^{-x_{j}\left(a+b \sin \left(N_{r} \mu_{1}-\rho_{j}\right)\right.} b N_{r} \cos \left(N_{r} \mu_{1}-\rho_{1}\right)\right. \\
& \left.-e^{-x_{j}\left(a+b \sin \left(N_{r} \mu_{1}-\rho_{j}\right)\right.} x_{j}\left(a+b \sin \left(N_{r} \mu_{1}-\rho_{j}\right)\right)^{2}\right] \\
& +\left(a+b \sin \left(N_{r} \mu_{1}-\rho_{j}\right)\right)^{-2} x_{j} \mu_{2} \\
& b N_{r} \cos \left(N_{r} \mu_{1}-\rho_{1}\right)+x_{j} \mu_{2}\left(a+b \sin \left(N_{r} \mu_{1}-\rho_{j}\right)\right)^{-1} \\
& b N_{r}^{2} \sin \left(N_{r} \mu_{1}-\rho_{1}\right) \\
\frac{\partial \dot{x}_{j}}{\partial \mu_{2}}= & -x_{j}\left(a+b \sin \left(N_{r} \mu_{1}-\rho_{j}\right)\right)^{-1} \\
& b N_{r} \cos \left(N_{r} \mu_{1}-\rho_{1}\right)
\end{aligned}
$$




\section{Capítulo 6}

\section{Aplicación al MSIP}

\subsection{Introducción}

Los motores sincrónicos de imán permanente (MSIP) se emplean como servo accionamientos en aplicaciones tales como equipamiento para periféricos de computadoras, robótica, y como accionamientos de velocidad ajustable en varias aplicaciones tales como bombas de calor, grandes ventiladores y compresores [79], [82], [85]. Algunos trabajos en los cuales se pueden encontrar ejemplos de aplicación son: [67] donde se emplea un MSIP como accionamiento para un manipulador robótico, [14] donde se lo utiliza en un vehículo de propulsión eléctrica. La aplicación en sistemas de movimiento a manivela y la comparación con otros motores de alterna se encuentra en [33]. Una buena descripción de la característica de las aplicaciones donde este motor puede ser usado está en [94]. Otro trabajo interesante es [52] donde se trata con los accionamientos de velocidad ajustable en diferentes aplicaciones en la industria del acero. Aunque la mayoría emplean el motor de inducción, en este trabajo se concluye que dado el estado actual del arte es importante que se investigue la factibilidad de aplicación de estos motores. 
Figura 6.1: Corte transversal del motor

\subsection{Aplicación al MSIP}

\subsubsection{El MSIP}

La principal virtud del MSIP es que puede funcionar en un amplio rango de velocidades [79]. El estator de la máquina es cilíndrico y alimentado por un bobinado trifásico, que a su vez es excitado por un inversor de transistores de alta frecuencia de conmutación. El rotor es de polos salientes con imán permanente construído con materiales magnético de tierras raras de alta permeabilidad. En la figura 6.1 se representa un corte transversal de la máquina en forma esquemática. Existen varios tipos de máquinas ya que el número de polos del rotor es variable. En la figura, el rotor presenta un único par de polos. El inversor que controla el accionamiento 
emplea técnicas de PWM [45] para generar corrientes que son funciones sinusoidales de la posición del rotor. Esto se hace para lograr una cupla constante en función de la posición angular del rotor. Por este motivo, para calcular las referencias del inversor se utiliza un codificador de posición angular ("resolver" o un "encoder") como sensor para la posición del rotor.

El estator del MSIP es alimentado por una tensión trifásica, equilibrada y simétrica con desfase de $120^{\circ}$. A cada tensión de fase la denominaremos $v_{a}, v_{b}$ y $v_{c}$, respectivamente. Sin embargo, expresaremos el modelo dinámico en el cuadro de referencia estacionario de dos ejes [56], [79]. La idea, muy empleada en teoría de máquinas, es usar una transformación 3 a 2 para crear dos tensiones ficticias $\left(v_{\alpha} \mathrm{y} v_{\beta}\right)$, relacionadas con las verdaderamente aplicadas de la siguiente manera:

$$
\begin{aligned}
v_{\alpha} & =\frac{2}{3} v_{a}-\frac{1}{3} v_{b}-\frac{1}{3} v_{c} \\
v_{\beta} & =\frac{1}{\sqrt{3}} v_{b}-\frac{1}{\sqrt{3}} v_{c}
\end{aligned}
$$

La misma transformación da origen a corrientes ficticias $\left(x_{\alpha}\right.$ y $\left.x_{\beta}\right)$ que están relacionadas con las corrientes de fase $\left(i_{a}, i_{b}\right.$ y $\left.i_{c}\right)$ por:

$$
\begin{aligned}
& x_{\alpha}=\frac{2}{3} i_{a}-\frac{1}{3} i_{b}-\frac{1}{3} i_{c} \\
& x_{\beta}=\frac{1}{\sqrt{3}} i_{b}-\frac{1}{\sqrt{3}} i_{c}
\end{aligned}
$$

En estas condiciones, el modelo dinámico viene dado por: [56]

$$
\begin{aligned}
\dot{\theta} & =\omega \\
\dot{\omega} & =\frac{-3}{2} \frac{\phi}{J} x_{\alpha} \sin \left(N_{r} \theta\right)+\frac{3}{2} \frac{\phi}{J} x_{\beta} \cos \left(N_{r} \theta\right)-\frac{B}{J} \omega \\
\dot{x}_{\alpha} & =\frac{-R}{L} x_{\alpha}+\frac{\phi}{L} \omega \sin \left(N_{r} \theta\right)+\frac{v_{\alpha}}{L} \\
\dot{x}_{\beta} & =\frac{-R}{L} x_{\beta}-\frac{\phi}{L} \omega \cos \left(N_{r} \theta\right)+\frac{v_{\beta}}{L}
\end{aligned}
$$

donde $x_{\alpha}, x_{\beta}, v_{\alpha}, v_{\beta}, \phi, R, L$ son las corrientes, tensiones, constante de flujo del imán permanente, resistencia e inductancia, respectivamente, y $\theta, \omega, B$, y $J$ son la posición del rotor, su velocidad angular, coeficiente de roce viscoso y momento 
de inercia del conjunto rotor/carga, respectivamente. $N_{r}$ es el número de pares de polos, que sin pérdida de generalidad supondremos igual a 1.

\section{La cupla generada por el MSIP}

De la ecuación (6.6) puede verse claramente, que la cupla generada $\left(T_{g}\right)$ por el MSIP, considerando $N_{r}=1$, es:

$$
T_{g}=\frac{-3}{2} \phi x_{\alpha} \sin \theta+\frac{3}{2} \phi x_{\beta} \cos \theta
$$

Debido a que el correcto funcionamiento del accionamiento exige que en el estado permanente la cupla generada sea constante es necesario sincronizar las llaves del inversor con la posición del rotor. Lo que normalmente se hace, es colocar un controlador de corriente cuya referencia es: $x_{\alpha}^{*}=-I \sin (\theta)$ y $x_{\beta}^{*}=I \cos (\theta)$ donde $I$ es constante. Cuando $x_{\alpha}=x_{\alpha}^{*}$ y $x_{\beta}=x_{\beta}^{*}$ se obtiene $T_{g}=\frac{3}{2} \phi I$.

Nota: Volveremos a analizar esto en el próximo capítulo donde trataremos con el control de velocidad del MSIP sin utilizar sensores óptico/mecánicos.

\subsubsection{Un observador para el MSIP}

Diferentes propuestas han sido realizadas en la literatura para estimar la posición y/o la velocidad angular del rotor del MSIP. Las principales son las ya descriptas en la subsección (3.4.2) (ver pág.34). En esta subsección proponemos utilizar un observador no lineal de orden reducido para estimar la posición y la velocidad angular del MSIP. El método empleado para construir el observador se basa en la técnica descripta en el Lema 4.4 (ver pág.48). Con el propósito de hacer más claro el desarrollo, introducimos aquí una leve modificación. Para construir el observador usaremos en el término de corrección las derivadas de las corrientes $\left(w=\left[\begin{array}{ll}\dot{x}_{\alpha} & \dot{x}_{\beta}\end{array}\right]^{\prime}\right)$, que no son directamente medidas, en lugar de las corrientes $\left(x_{\alpha}, x_{\beta}\right)$. Más adelante (ver pág.101), produciremos las modificaciones necesarias para escribir las ecuaciones que describen al observador en función de las mediciones. Partiremos al vector de 
estado del sistema (6.5-6.8) en dos partes, una que contiene las variables a estimar $(\theta$ y $\omega)$ y la otra la que contiene a las variables $x_{\alpha}$ y $x_{\beta}$ que no deseamos estimar porque son una combinación conocida de las mediciones. Proponemos como observador al sistema :

$$
\left[\begin{array}{c}
\dot{\hat{\theta}} \\
\dot{\hat{\omega}}
\end{array}\right]=\left[\begin{array}{l}
\hat{\omega} \\
\hat{a}
\end{array}\right]+G(\hat{\theta}, \hat{\omega})(w-\hat{w})
$$

con $\hat{a}=\frac{-3}{2} \frac{\phi}{J} x_{\alpha} \sin (\hat{\theta})+\frac{3}{2} \frac{\phi}{J} x_{\beta} \cos (\hat{\theta})-\frac{B}{J} \hat{\omega}$, donde $\hat{\theta}, \hat{\omega}$ son la posición angular del rotor y su velocidad estimadas. Para construir el término de corrección se emplean

$$
w=\left[\begin{array}{c}
\frac{-R}{L} x_{\alpha}+\frac{\phi}{L} \omega \sin \theta+\frac{v_{\alpha}}{L} \\
\frac{-R}{L} x_{\beta}-\frac{\phi}{L} \omega \cos \theta+\frac{v_{\beta}}{L}
\end{array}\right]
$$

$\mathrm{y}$

$$
\hat{w}=\left[\begin{array}{c}
\frac{-R}{L} x_{\alpha}+\frac{\phi}{L} \hat{\omega} \sin \hat{\theta}+\frac{v_{\alpha}}{L} \\
\frac{-R}{L} x_{\beta}-\frac{\phi}{L} \hat{\omega} \cos \hat{\theta}+\frac{v_{\beta}}{L}
\end{array}\right]
$$

La matriz $G(\hat{\theta}, \hat{\omega})$ debe ser diseñada para garantizar la convergencia del observador. Para ello, procedemos de la siguiente manera. Está claro que (6.11) no es lineal en $\theta$ y $\omega$. Introducimos la transformación de coordenadas

$$
z=T(\theta, \omega)=\left[\begin{array}{c}
T_{1}(\theta, \omega) \\
T_{2}(\theta, \omega)
\end{array}\right]=\left[\begin{array}{c}
z_{1} \\
z_{2}
\end{array}\right]=\left[\begin{array}{c}
\frac{\phi}{L} \omega \sin (\theta) \\
-\frac{\phi}{L} \omega \cos (\theta)
\end{array}\right]
$$

Nota: La no globalidad de la transformación no genera más inconvenientes que el problema de la distinguibilidad expuesto en la subsección (6.3.1) (ver pág.113)

Si usamos (6.12), podemos escribir (6.11) como:

$$
w=\left[\begin{array}{c}
z_{1}+u_{\alpha} \\
z_{2}+u_{\beta}
\end{array}\right]=z+u
$$

con $z=\left[\begin{array}{ll}z_{1} & z_{2}\end{array}\right]^{T}$ y $u=\left[\begin{array}{ll}u_{\alpha} & u_{\beta}\end{array}\right]^{T}$, donde

$$
u_{\alpha}=\frac{v_{\alpha}-R x_{\alpha}}{L} \quad u_{\beta}=\frac{v_{\beta}-R x_{\beta}}{L}
$$


son funciones de las variables medidas $x_{\alpha}, x_{\beta}, v_{\alpha} \mathrm{y} v_{\beta}, \mathrm{y}$ de parámetros conocidos $(\mathrm{R}$ y L). Reemplazando los valores de $\omega, \theta$ y sus derivadas de (6.5-6.8) en $\dot{z}$, obtenemos en las coordenadas transformadas $z$ :

$$
\dot{z}=A z+\rho(z, i)
$$

donde

$$
\begin{gathered}
A=\left[\begin{array}{cc}
\frac{-B}{J} & 0 \\
0 & \frac{-B}{J}
\end{array}\right] \\
\rho(z, i)=\left[\begin{array}{c}
\frac{K k_{\alpha} z_{1}^{2}-K k_{\beta} z_{1} z_{2}}{z_{1}^{2}+z_{2}^{2}}-\sqrt{z_{1}^{2}+z_{2}^{2}} \frac{z_{2}}{K} \\
\frac{-K k_{\beta} z_{2}^{2}+K k_{\alpha} z_{1} z_{2}}{z_{1}^{2}+z_{2}^{2}}+\sqrt{z_{1}^{2}+z_{2}^{2}} \frac{z_{1}}{K}
\end{array}\right]
\end{gathered}
$$

$\operatorname{con} K=\frac{\phi}{L}, k_{\alpha}=\frac{-3 \phi}{2 J} x_{\alpha}, k_{\beta}=\frac{3 \phi}{2 J} x_{\beta}, i=\left[\begin{array}{ll}x_{\alpha} & x_{\beta}\end{array}\right]^{T}$.

En las coordenadas $z$, el observador propuesto viene dado por:

$$
\begin{aligned}
\dot{\hat{z}} & =A \hat{z}+\rho(\hat{z}, i)+G(w-\hat{w}) \\
\hat{w} & =\hat{z}+u
\end{aligned}
$$

donde ahora,

$$
G=\left[\begin{array}{ll}
g_{11} & g_{12} \\
g_{21} & g_{22}
\end{array}\right]
$$

es una matriz constante.

El sistema en las coordenadas transformadas (6.13) no tiene la forma de Brunovsky, pero esto no importa, pues las matrices $A$ y $C=I$ forman un par observable, como se desprende del análisis de la matriz de observabilidad

$$
\left[\begin{array}{l}
I \\
A
\end{array}\right]
$$


o algún otro test que se quiera aplicar. Por lo tanto, si se desea se puede pasar de una a otra forma con adecuadas transformaciones lineales. Si se puede elegir $G$ tal como fue hecho en la demostración del Lema 4.4 (ver pág.48), el observador dado por (6.14) es asintóticamente estable. Además, la convergencia del error a cero es exponencial.

Consideremos el error de observación dado por $e=z-\hat{z}$, entonces

$$
\dot{e}=A_{c} e+\Delta \gamma
$$

donde en este caso particular, la ecuación (4.31) tiene $A_{c}=A-G$, ya que $C=I$ y $\Delta \gamma=\gamma(z, i)-\gamma(\hat{z}, i)$. Por lo tanto, en este caso la ecuación (4.32) tiene la forma de (6.18).

$$
\dot{V}=e^{\prime}\left[(A-G)^{\prime} P+P(A-G)\right] e+(\Delta \gamma)^{\prime} P e+e^{T} P \Delta \gamma
$$

Si $\gamma(z, i)$ satisface la condición de Lipschitz en la primer variable, uniformemente en la segunda, en un compacto, con constante $\Gamma$ (ver 4.23, en pág 46), entonces:

$$
\dot{V} \leq-q\|e\|^{2}+2 p \Gamma\|e\|^{2}
$$

como en (4.33); donde $q$ es el mínimo autovalor de $Q, p$ es el máximo autovalor de $P$. De (6.19) puede verse que si podemos encontrar $p, q$ y $\Gamma$ tales que $\sigma=-q+2 p \Gamma<0$ luego el punto de equilibrio $(e=0)$ es asintóticamente estable. Mas aún, ya que

$$
V(t) \leq V\left(t_{0}\right) e^{\frac{\sigma}{p}\left(t-t_{0}\right)}
$$

la convergencia es exponencial, es decir $\|e(t)\| \leq\left\|e\left(t_{0}\right)\right\| e^{\frac{\sigma}{2 p}\left(t-t_{0}\right)}$. La ecuación (6.19) debe ser usada para encontrar la matriz constante $G$. Después de calcular $\Gamma$, debemos elegir los valores de $P, Q$ y $G$ tal que $-q+2 p \Gamma<0$.

Veamos ahora la forma que toma el observador. Una vez elegida $G$, ya que $\hat{z}=T(\hat{\theta}, \hat{\omega})$, entonces:

$$
\dot{\hat{z}}=\Upsilon(\hat{\theta}, \hat{\omega})\left[\begin{array}{l}
\dot{\hat{\theta}} \\
\dot{\hat{\omega}}
\end{array}\right]
$$


siendo $\Upsilon(\hat{\theta}, \hat{\omega})$ el Jacobiano de $T$. En las coordenadas originales, (6.14) está dada por:

$$
\left[\begin{array}{l}
\dot{\hat{\theta}} \\
\dot{\hat{\omega}}
\end{array}\right]=\left[\begin{array}{l}
\hat{\omega} \\
\hat{a}
\end{array}\right]+\Upsilon^{-1} G(w-\hat{w})
$$

con

$$
\Upsilon=\left[\begin{array}{cc}
\frac{\phi}{L} \hat{\omega} \cos (\hat{\theta}) & \frac{\phi}{L} \sin (\hat{\theta}) \\
\frac{\phi}{L} \hat{\omega} \sin (\hat{\theta}) & -\frac{\phi}{L} \cos (\hat{\theta})
\end{array}\right]
$$

Claramente, puede verse que la ganancia no lineal del observador $G(\hat{\theta}, \hat{\omega})$ en $(6.10)$ queda dada por el producto de la matriz constante $G$ y la inversa de la matriz del Jacobiano de la transformación evaluada en las variables estimadas $\Upsilon(\hat{\theta}, \hat{\omega})$.

\section{Una posible elección de la matriz constante G}

Supongamos que se eligen $g_{12}=0$ y $g_{21}=0$, entonces $A_{c}$ es una matriz diagonal dada por:

$$
A_{c}=A-G=\left[\begin{array}{cc}
\frac{-B}{J}-g_{11} & 0 \\
0 & \frac{-B}{J}-g_{22}
\end{array}\right]
$$

Podemos elegir $P$ igual a la matriz identidad y $Q$ igual a una matriz diagonal

$$
Q=\left[\begin{array}{cc}
q_{11} & 0 \\
0 & q_{11}
\end{array}\right]
$$

tal que la solución de

$$
(A-G)^{\prime} P+P(A-G)=-Q
$$

es $2\left(\frac{B}{J}+g_{11}\right)=q_{11}$ y $2\left(\frac{B}{J}+g_{22}\right)=q_{11}$, entonces $g_{11}=g_{22}$. En consecuencia, obtenemos $p=1$ y $q=2\left(\frac{B}{J}+g_{11}\right)$. Luego debe cumplirse que $q>2 p \Gamma$ lo que siempre puede lograrse eligiendo convenientemente $g_{11}>\Gamma-\frac{B}{J}$. Más aún, se ve 
claramente cómo una elección "sobrada" de $g_{11}$, deja un margen para variaciones de $\frac{B}{J}$ por sobre sus valores nominales. Entonces $\sigma=-2\left(\frac{B}{J}+g_{11}\right)+2 \Gamma$ acota la velocidad de convergencia.

\section{Estructura del observador}

El observador en (6.21) emplea en el término de corrección las derivadas de las corrientes medidas. Esto puede ser un inconveniente, ya que calcular las derivadas a partir de la medición puede dar una estimación muy ruidosa. Por este motivo, proponemos una estructura para el observador que evita el tener que derivar.

Reescribimos (6.21) como:

$$
\left[\begin{array}{c}
\dot{\hat{z}}_{1} \\
\dot{\hat{z}}_{2}
\end{array}\right]-G w=\Upsilon\left[\begin{array}{c}
\hat{\omega} \\
\hat{a}
\end{array}\right]-G \hat{w}
$$

Si utilizamos la siguiente transformación:

$$
\left[\begin{array}{c}
\dot{\hat{z}}_{1} \\
\dot{\hat{z}_{2}}
\end{array}\right]-G w=\left[\begin{array}{c}
\dot{\nu_{1}} \\
\dot{\nu_{2}}
\end{array}\right]
$$

(6.25) se transforma en:

$$
\left[\begin{array}{c}
\dot{\nu_{1}} \\
\dot{\nu_{2}}
\end{array}\right]=\Upsilon\left[\begin{array}{l}
\hat{\omega} \\
\hat{a}
\end{array}\right]-G \hat{w}
$$

Las ecuaciones para calcular $\hat{\theta}$ y $\hat{\omega}$ se obtienen de la siguiente forma. Si integramos (6.26) entonces:

$$
\left[\begin{array}{c}
\hat{z}_{1} \\
\hat{z}_{2}
\end{array}\right]=\left[\begin{array}{c}
\nu_{1}+g_{11} x_{\alpha}+g_{12} x_{\beta} \\
\nu_{2}+g_{21} x_{\alpha}+g_{22} x_{\beta}
\end{array}\right]
$$

Usando (6.12)

$$
\begin{gathered}
\hat{\theta}=\operatorname{tg}^{-1}\left(\frac{\hat{z}_{1}}{-\hat{z}_{2}}\right) \\
\hat{\omega}=\frac{L}{\phi} \sqrt{\hat{z}_{1}^{2}+\hat{z}_{2}^{2}}
\end{gathered}
$$


entonces, reemplazando (6.28) en (6.29) obtenemos:

$$
\begin{aligned}
\hat{\theta} & =t g^{-1}\left[\frac{\left(\nu_{1}+g_{11} x_{\alpha}+g_{12} x_{\beta}\right)}{-\left(\nu_{2}+g_{21} x_{\alpha}+g_{22} x_{\beta}\right)}\right] \\
\hat{\omega} & =\frac{L}{\phi} \sqrt{\left(\nu_{1}+g_{11} x_{\alpha}+g_{12} x_{\beta}\right)^{2}+\left(\nu_{2}+g_{21} x_{\alpha}+g_{22} x_{\beta}\right)^{2}}
\end{aligned}
$$

De este modo, las ecuaciones que representan el observador son (6.27), (6.31) y (6.32).

Nota: Remarcamos que la construcción del observador requiere la integración en tiempo real de las ecuaciones diferenciales (6.27). Debe notarse que las ecuaciones (6.31) y (6.32) pueden incorporarse fácilmente en una rutina de integración numérica.

\subsection{Resultados de simulación}

En esta sección mostramos la simulación para un MSIP en un sistema a lazo cerrado. Las señales de la posición del rotor y la velocidad empleadas para la realimentación son reemplazados por los valores estimados.

El MSIP considerado tiene los datos y parámetros dados en la siguiente tabla:

\begin{tabular}{||c|c||}
\hline$P_{N}$ & $600 \mathrm{~W}$ \\
$\Omega_{N}$ & $200 \mathrm{rad} / \mathrm{seg}$. \\
pares de polos & 1 \\
$L$ & $20.5 \mathrm{mHy}$ \\
$R$ & $1.55 \Omega$ \\
$\phi$ & $0.22 \mathrm{Nm} / \mathrm{A}$ \\
$J$ & $2.210^{-3} \mathrm{Kgm}^{2}$ \\
$B$ & $2.210^{-3} \mathrm{Nms}_{\mathrm{rad}}$ \\
\hline
\end{tabular}

Los límites del accionamiento son: $V_{\max }=300 \mathrm{~V} ; I_{\max }=20 \mathrm{~A}$. La figura 6.2 ilustra las conexiones del sistema para la simulación. El esquema de control, que es de uso clásico en accionamientos, consta de un controlador proporcional+integral 
Figura 6.2: Diagrama en bloques de la conexión del accionamiento

(PI) [111] usado para seguir una referencia de velocidad $\omega^{\star}$, dado por $K_{p}\left(\omega^{\star}-\hat{\omega}\right)+$ $\frac{K_{p}}{T_{i}} \int\left(\omega^{\star}-\hat{\omega}\right) d t$, con $K_{p}=0.5$ y $T_{i}=0.1 \mathrm{seg}$.

Nota: En este punto cabe destacar que hasta este momento, no se ha realizado ningún estudio que garantice la estabilidad del funcionamiento en lazo cerrado del sistema con observador y controlador. Está claro, que a diferencia del caso lineal, no alcanza para garantizar la estabilidad del sistema no lineal a lazo cerrado, el diseño de un observador que converge y un controlador estabilizante [30]. En otras palabras, no pueden aplicarse directamente el teorema de separación y el principio de equivalencia cierta [19], [51] como en los sistemas lineales. En el próximo capítulo analizaremos un esquema de control de lazo cerrado usando observador para el MSIP para el que se garantiza estabilidad. 
Figura 6.3: Velocidad del motor durante el arranque, para distintos valores de la posición inicial del observador mientras que su velocidad inicial fue siempre de $30 \mathrm{rad} / \mathrm{seg}$.

El controlador de velocidad recibe el valor de la velocidad estimada del observador y calcula el valor de corriente que requiere el motor. Con el valor de referencia de la corriente y el valor de la posición estimada del observador, se calculan los valores de tensiones necesarios para alimentar el motor. Siguiendo las consideraciones de diseño propuestas obtenemos los siguientes valores de ganancias $g_{11}=g_{22}=500$ y $g_{12}=g_{21}=0$.

La figura 6.3 ilustra la evolución de la velocidad durante el transitorio de arranque cuando el valor de la velocidad de referencia es de $150 \mathrm{rad} / \mathrm{seg}$. Se muestran la velocidad real del motor cuando la velocidad y la posición estimadas son utilizadas 
Figura 6.4: Error de posición durante el arranque. Posición inicial del rotor $0^{\circ}$. Posición inicial del observador - - - $80^{\circ} ; \cdots 40^{\circ} ;---40^{\circ} ;-\quad-80^{\circ}$

para alimentar los controladores del accionamiento. Diferentes curvas en la figura 6.3 corresponden a diferentes valores de la posición inicial estimada entre $-80^{\circ} \mathrm{y}$ $80^{\circ}$ y el valor de la velocidad inicial estimada es $30 \mathrm{rad} / \mathrm{seg}$. En las figuras 6.4 y 6.5 se presentan los errores de posición y velocidad, repectivamente.

En las figuras puede verse como el error converge a cero. Las figuras 6.6-6.11 muestran cual es el comportamiento del observador ante la presencia de incertidumbres en los parámetros mecánicos y perturbaciones de cupla. Se suponen incertidumbres en la inercia del rotor $(J)$ y en el coeficiente de amortiguamiento $(B)$. Las figuras 6.6 y 6.7 ilustran el error de la posición del rotor y el error de la velocidad para un valor de $B=100 \%$ mayor que el nominal y $50 \%$ menor que el nominal. 
Figura 6.5: Error de velocidad durante el arranque. Posición inicial del rotor $0^{\circ}$. Posición inicial del observador $-\cdot-80^{\circ} ; \cdots 40^{\circ} ;---40^{\circ} ;-\quad-80^{\circ}$. Velocidad inicial del motor $0 \mathrm{rad} / \mathrm{seg}$. Velocidad inicial del observador: $30 \mathrm{rad} / \mathrm{seg}$. 
Figura 6.6: Error de posición debido a incertidumbres en $B$

Puede verse que el error de posición es despreciable y el error en la velocidad con el motor corriendo a velocidad nominal (150 rad/seg) es menor que $0.2 \%$.

En las figuras 6.8 y 6.9 se representan los errores en la posición y la velocidad cuando el momento de inercia es $J=100 \%$ mayor y $50 \%$ menor que el valor nominal. Como era de esperar la incertidumbre en $J$ no afecta el valor de estado estacionario en la estimación. Este sólo modifica el comportamiento transitorio del observador, pero no compromete su estabilidad.

Finalmente, se aplica al motor una cupla de carga no modelada de valor $3 \mathrm{Nm}$ mientras el motor está funcionando a $150 \mathrm{rad} / \mathrm{seg}$. En las figuras 6.10 y 6.11 se muestran los resultados. Como era de esperarse, la perturbación hace que el error de velocidad se incremente, pero éste es menos que $2 \%$ de la velocidad de fun- 
Figura 6.7: Error de velocidad debido a incertidumbres en $B$ 
Figura 6.8: Error de posición debido a incertidumbres en $J .--100 \%$ mayor; $50 \%$ menor 
Figura 6.9: Error de velocidad debido a incertidumbres en $J$ 
Figura 6.10: Error de posición frente a una variación de cupla 
Figura 6.11: Error de velocidad frente a una variación de cupla 
cionamiento. También, aparece un error en la posición estimada de menos de $2^{\circ}$.

Es claro, que el observador permanece estable, aún cuando existan algunos desajustes entre los parámetros mecánicos reales y los del modelo. Sin embargo, si no pueden tolerarse los errores que aparecen, puede emplearse un esquema adaptivo por extensión similar al utilizado en el capítulo anterior.

\subsubsection{Algunas consideraciones adicionales}

\section{El problema de la distinguibilidad}

La transformación (6.12) no es global ya que los puntos $(\theta, \omega)$ y $(\theta+\pi,-\omega)$ son transformados al mismo punto $\left(z_{1}, z_{2}\right)$. Sin embargo, mirando las ecuaciones del modelo notamos que debido a que $\omega \sin \theta=-\omega \sin (\theta+\pi)$ y $\omega \cos \theta=-\omega \cos (\theta+\pi)$ no se puede distinguir entre los estados $\left[\begin{array}{ll}\theta & \omega\end{array}\right]^{\prime}$ y $\left[\begin{array}{ll}\theta+\pi & -\omega\end{array}\right]^{\prime}$ en las ecuaciones de corriente (6.7) y (6.8). Por lo tanto, existe un problema de distinguibilidad para estos estados al usar las mediciones de corriente o sus derivadas. Por esto es que cualquier técnica que se use para construir un observador con sólo la medición de las corrientes, no podrá ser global. Por ende, la restricción sobre la transformación propuesta no implica una limitación adicional para la "globalidad" que la ya impuesta por problemas de indistiguibilidad. Este inconveniente que restringe la globalidad se manifiesta independientemente de la técnica que utilicemos para construir el observador. Por ejemplo, el problema también aparece en [4] donde se propone emplear un Filtro de Kalman Extendido, usando la medición de las corrientes. En este trabajo se propone un algoritmo para solucionarlo. Se compara el signo de la velocidad estimada con el de la derivada de la posición estimada, si los signos son diferentes se suma $\pi$ a la posición estimada. Este sencillo algoritmo puede ser empleado en nuestro caso, ya que es independiente del método utilizado para la estimación de la velocidad y la posición. Sin embargo, la convergencia y la velocidad de convergencia no están garantizadas.

Cabe aclarar que para este motor, se puede construir un observador global con 
dinámica lineal del error si se tiene la medición de posición [109]. Sin embargo, resaltamos que nuestro objetivo es no usar el sensor opto/mecánico de posición por las razones expuestas en el Capítulo 1.

\section{Aspectos de implementación}

Como en el caso del MRC realizaremos una estimación de la capacidad de cómputo necesaria suponiendo que se emplea un algoritmo del tipo Runge-Kutta [95]. Para calcular los valores de la derivada en un instante de tiempo es necesario realizar 9 sumas, 20 multiplicaciones y 1 división. Además es necesario calcular seno, coseno, raíz cuadrada y arco tangente y resolver dos ecuaciones diferenciales por el método de Runge-Kutta de 4to. orden.

Considerando que para el seno, el coseno, la raíz cuadrada y el arco tangente se utilizan 10 operaciones, el total de operaciones necesarias es menor que que 250 . Con los valores habituales de los motores, se puede estimar que un tiempo de muestreo de $100 \mu$ seg es aceptable. Entonces, considerando, que un DSP realiza una operación por ciclo, son necesarias $2.5 \mathrm{M}$ flops/seg, lo que es fácilmente alcanzado por un DSP del tipo TMS320C30, por ejemplo.

\subsection{Conclusión}

En este capítulo presentamos un nuevo método para diseñar un observador no lineal de orden reducido para estimar la posición y la velocidad angular del rotor del MSIP. El término de corrección es una función no lineal de los estados estimados y el método de diseño es una aplicación de los resultados obtenidos en el Lema 4.4. Se logran condiciones suficientes empleando la teoría de Lyapunov, que permiten garantizar la convergencia a cero del error de estimación. Una adecuada elección de las matrices de la ecuación de Lyapunov permite un diseño sencillo del término de corrección. El mayor inconveniente consiste en calcular la constante de Lipschitz. 
Sin embargo, esto siempre puede hacerse si la función es derivable y se trabaja en un compacto.

Debido a que el observador está basado en un modelo paramétrico del motor, su comportamiento depende de la exactitud con que se conocen los valores de los parámetros. A pesar de ello, hemos mostrado que el observador presenta un buen desempeño, aunque se produzcan variaciones en los parámetros mecánicos. Por lo tanto, variaciones de cupla no modeladas, no introducen errores de importancia. Sin embargo, si en la aplicación bajo estudio las variaciones fueran intolerables para el desempeño buscado, se puede utilizar un esquema adaptivo con extensión de estados, similar al del capítulo anterior.

Como fue indicado en el capítulo 5, consideramos que se debe tomar en cuenta el comentario que se realiza, en las conclusiones finales de la tesis, respecto del empleo del PWM en el control de accionamientos. 


\section{Capítulo 7}

\section{Control de velocidad del MSIP}

\subsection{Introducción}

En la casi totalidad de las aplicaciones, el objetivo de emplear observadores es estimar los estados no medidos para poder utilizarlos en un esquema de control de lazo cerrado. En los sistemas lineales se sabe que es posible diseñar separadamente, un observador que converja y un controlador estabilizante, para que estos puedan ser incluídos juntos en un esquema de lazo cerrado. Este hecho es conocido como el teorema de separación y el principio de equivalencia cierta [51], [86]. Este principio no es válido en general, y entonces no podemos utilizarlo en un esquema de lazo cerrado en sistemas no lineales.

Esto obliga a realizar un estudio de la dinámica del sistema que considera en su conjunto a la planta, al controlador y al observador. Aplicaciones en las que observadores y controladores se juntan en un esquema de lazo cerrado pueden verse, entre otras, en [12], [18], [35]. En este capítulo proponemos un esquema de lazo cerrado que permite controlar la velocidad de un accionamiento que utiliza un motor sincrónico de imán permanente. La estrategia de control empleada es la linealización exacta por transformación y realimentación. La clara descripción de la estrategia y las condiciones que debe satisfacer un sistema para que la misma pueda emplearse, se encuentran en [24], [47], [88]. La aplicación de esta estrategia al MRC se encuentra en [20], [46] y al motor de paso de imán permanente en [12]. En estos casos, es necesario 
medir las variables mecánicas y las eléctricas para construir la estrategia de control. Como puede verse en la próxima sección, este es también el caso si se implementa esta estrategia en el MSIP. Para evitar el uso de los sensores mecánico/ópticos, una posibilidad es construir una estrategia que utilice un observador para estimar las variables mecánicas.

\subsection{La estrategia de control}

Entre las técnicas desarrolladas para el diseño de estrategias de control para los sistemas no lineales, la linealización exacta por transformación y realimentación ha tenido amplia difusión en los últimos años. La idea consiste en encontrar por medio de transformación de coordenadas y realimentación, una descripción entrada/salida lineal del sistema y entonces aplicar en las nuevas coordenadas bien conocidas leyes de control lineal. En esta sección describiremos cómo emplear esta estrategia en el caso del motor sincrónico de imán permanente.

Recordemos que el modelo del MSIP, en su marco estacionario de referencia de dos ejes, está dado por:

$$
\begin{aligned}
\dot{\theta} & =\omega \\
\dot{\omega} & =\frac{-3}{2} \frac{\phi}{J} x_{\alpha} \sin \theta+\frac{3}{2} \frac{\phi}{J} x_{\beta} \cos \theta-\frac{B}{J} \omega \\
\dot{x}_{\alpha} & =\frac{-R}{L} x_{\alpha}+\frac{\phi}{L} \omega \sin \theta+\frac{v_{\alpha}}{L} \\
\dot{x}_{\beta} & =\frac{-R}{L} x_{\beta}-\frac{\phi}{L} \omega \cos \theta+\frac{v_{\beta}}{L}
\end{aligned}
$$

donde $x_{\alpha}, x_{\beta}, v_{\alpha}, v_{\beta}, R, L$ son las corrientes, tensiones, resistencia e inductancia, respectivamente, y $\theta, \omega, B$ y $J$ son la posición del rotor, su velocidad angular, el coeficiente viscoso y el momento de inercia, respectivamente. Al sistema (7.1-7.4) le podemos aplicar la transformación, no singular, dada por:

$$
\left[\begin{array}{l}
v_{d} \\
v_{q}
\end{array}\right]=\left[\begin{array}{cc}
\cos \theta & \sin \theta \\
-\sin \theta & \cos \theta
\end{array}\right]\left[\begin{array}{l}
v_{\alpha} \\
v_{\beta}
\end{array}\right]
$$


Figura 7.1: Esquema de la trasformación D-Q

$$
\left[\begin{array}{l}
x_{d} \\
x_{q}
\end{array}\right]=\left[\begin{array}{cc}
\cos \theta & \sin \theta \\
-\sin \theta & \cos \theta
\end{array}\right]\left[\begin{array}{l}
x_{\alpha} \\
x_{\beta}
\end{array}\right]
$$

Esta transformación es conocida con el nombre de transformación $D-Q$ en el ámbito de la electrónica de potencia y es empleada en el estudio de diferentes tipos de accionamientos. Una representación esquemática de esta transformación es la dada en la figura 7.1. El cuadro de referencia de dos ejes $\left(\begin{array}{l}\alpha \\ \text { y }\end{array} \beta\right)$ está estacionario, mientras los ejes ortogonales $D$ y $Q$ giran con el rotor. Reemplazando (7.5) y (7.6) en (7.1-7.4) se obtiene:

$$
\begin{aligned}
\dot{\theta} & =\omega \\
\dot{\omega} & =\frac{3}{2} \frac{\phi}{J} x_{q}-\frac{B}{J} \omega
\end{aligned}
$$




$$
\begin{aligned}
& \dot{x}_{d}=\frac{-R}{L} x_{d}+\omega x_{q}+\frac{v_{d}}{L} \\
& \dot{x}_{q}=\frac{-R}{L} x_{q}-\omega x_{d}-\frac{\phi}{L} \omega+\frac{v_{q}}{L}
\end{aligned}
$$

El sistema en las nuevas coordenadas, ecuaciones (7.7)-(7.10) también es no lineal, pero puede definirse una entrada auxiliar $\left[\begin{array}{ll}u_{d} & u_{q}\end{array}\right]^{\prime}$, tal que:

$$
\begin{aligned}
& v_{d}=R x_{d}-L \omega x_{q}+L u_{d} \\
& v_{q}=R x_{q}+L \omega x_{d}+\phi \omega+L u_{q}
\end{aligned}
$$

Si reemplazamos (7.11) y (7.12) en (7.9) y (7.10), respectivamente, obtenemos:

$$
\begin{aligned}
\dot{\theta} & =\omega \\
\dot{\omega} & =\frac{3}{2} \frac{\phi}{J} x_{q}-\frac{B}{J} \omega \\
\dot{x}_{d} & =u_{d} \\
\dot{x}_{q} & =u_{q}
\end{aligned}
$$

Nota: El cálculo de la transformación utilizando las adecuadas herramientas matemáticas se encuentra en el Apéndice 7.A.

Como puede verse en (7.13)-(7.16) se ha logrado representar al sistema en nuevas coordenadas, $\left[\begin{array}{llll}\theta & \omega & x_{d} & x_{q}\end{array}\right]^{\prime}$, como dos sub-sistemas lineales desacoplados, uno de tercer orden, con variables de estado $\theta, \omega, x_{q}$ y entrada $u_{q}$, y otro de primer orden, con variable $x_{d}$ y entrada $u_{d}$. Dado que en las nuevas coordenadas el sistema con entradas $u_{d}$ y $u_{q}$ es lineal, ahora es posible diseñar estrategias lineales para el lazo externo de control. Cabe destacar que para construir esta estrategia de control es necesario contar con las cuatro variables de estado, $\left[\begin{array}{llll}\theta & \omega & x_{\alpha} & x_{\beta}\end{array}\right]^{\prime}$, para implementar las ecuaciones (7.5), (7.6), (7.11) y (7.12). Por lo tanto, para construir la realimentación es necesario utilizar cuatro medidores de los cuales dos son para las variables mecánicas. Sin embargo, si deseamos evitar la medición de las variables mecánicas podemos desarrollar una estrategia de control basada en la linealización exacta por transformación y realimentación utilizando estimaciones de las mismas. 


\subsection{Control de velocidad sin sensores mecánicos}

Basados en las consideraciones de la sección anterior, proponemos un esquema de control de velocidad para el MSIP que emplea la linealización exacta por transformación y realimentación usando las estimaciones de las variables mecánicas obtenidas a partir del observador del capítulo 6 .

Después de utilizar la transformación $D-Q$ obtuvimos:

$$
\begin{aligned}
\dot{\theta} & =\omega \\
\dot{\omega} & =\frac{3}{2} \frac{\phi}{J} x_{q}-\frac{B}{J} \omega \\
\dot{x}_{d} & =\frac{-R}{L} x_{d}+\omega x_{q}+\frac{v_{d}}{L} \\
\dot{x}_{q} & =\frac{-R}{L} x_{q}-\omega x_{d}-\frac{\phi}{L} \omega+\frac{v_{q}}{L}
\end{aligned}
$$

Si utilizamos la ley de control dada por:

$$
\begin{aligned}
& v_{d}=R x_{d}-L \omega x_{q}+L u_{d} \\
& v_{q}=R x_{q}+L \omega x_{d}+\phi \omega+L u_{q}
\end{aligned}
$$

obtenemos el sistema lineal y desacoplado dado por (7.13)-(7.16). Este esquema de control, que no usa observador es representado en la figura 7.2. Sin embargo, nuestra propuesta es eliminar los sensores de las variables mecánicas y emplear en el lazo un observador para las mismas, tal como lo representamos en la figura 7.3. Debido al uso de las variables estimadas, la ley de control está dada por:

$$
\begin{aligned}
& \hat{v}_{d}=R \hat{x}_{d}-L \hat{\omega} \hat{x}_{q}+L u_{d} \\
& \hat{v}_{q}=R \hat{x}_{q}+L \hat{\omega} \hat{x}_{d}+\phi \hat{\omega}+L u_{q}
\end{aligned}
$$

Cuando se incluye el observador en el lazo, la dinámica del sistema puede ser descripta por:

$$
\begin{aligned}
\dot{\theta} & =\omega \\
\dot{\omega} & =\frac{3}{2} \frac{\phi}{J} x_{q}-\frac{B}{J} \omega \\
\dot{x}_{d} & =u_{d}+\varphi_{1} \\
\dot{x}_{q} & =u_{q}+\varphi_{2}
\end{aligned}
$$


Figura 7.2: Esquema de control sin observador 
Figura 7.3: Esquema de control con observador 
La aparición de las funciones no lineales $\varphi_{1}$ y $\varphi_{2}$, se debe a que las variables de control $\left(\left[\begin{array}{ll}v_{\alpha} & v_{\beta}\end{array}\right]\right)$ no toman los valores exactos necesarios para la linealización por transformación y realimentación, dados por (7.21) y (7.22), sino que toman los valores dados por (7.23) y (7.24) que se obtienen usando las variables estimadas. El valor de estas funciones es:

$$
\begin{aligned}
\varphi_{1} & =\frac{R}{L}\left(\hat{x}_{d}-x_{d}\right)-\left(\hat{\omega} \hat{x}_{q}-\omega x_{q}\right) \\
\varphi_{2} & =\frac{R}{L}\left(\hat{x}_{q}-x_{q}\right)+\left(\hat{\omega} \hat{x}_{d}-\omega x_{d}\right)+\frac{\phi}{L}(\hat{\omega}-\omega)
\end{aligned}
$$

Obviamente, cuando las variables estimadas coinciden con las verdaderas (error de estimación nulo), las funciones $\varphi_{1}$ y $\varphi_{2}$ se anulan, ya que $\left[\begin{array}{ll}\hat{v}_{d} & \hat{v}_{q}\end{array}\right]=\left[\begin{array}{ll}v_{d} & v_{q}\end{array}\right]$. Si se utiliza el observador propuesto en 6.2.2, (ver pág.97), la dinámica del error de estimación es:

$$
\begin{aligned}
\dot{e}_{\theta} & =e_{\omega}+\alpha_{1}\left(\theta, \omega, e_{\theta}, e_{\omega}\right) \\
\dot{e}_{\omega} & =\frac{-B}{J} e_{\omega}+\frac{3 \phi}{2 J}\left(x_{q}-\hat{x}_{q}\right)+\alpha_{2}\left(\theta, \omega, e_{\theta}, e_{\omega}\right)
\end{aligned}
$$

donde

$$
\begin{aligned}
\alpha_{1}= & \frac{\cos \left(\theta-e_{\theta}\right)}{\frac{\phi}{L}\left(\omega-e_{\omega}\right)}\left[g_{11} \frac{\phi}{L}\left(\omega \sin \theta-\left(\omega-e_{\omega}\right) \sin \left(\theta-e_{\theta}\right)\right)-\right. \\
& g_{12} \frac{\phi}{L}\left(\omega \cos \theta-\left(\omega-e_{\omega}\right) \cos \left(\theta-e_{\theta}\right)\right] \\
+ & \frac{\sin \left(\theta-e_{\theta}\right)}{\frac{\phi}{L}\left(\omega-e_{\omega}\right)}\left[g_{21} \frac{\phi}{L}\left(\omega \sin \theta-\left(\omega-e_{\omega}\right) \sin \left(\theta-e_{\theta}\right)\right)-\right. \\
& g_{22} \frac{\phi}{L}\left(\omega \cos \theta-\left(\omega-e_{\omega}\right) \cos \left(\theta-e_{\theta}\right)\right] \\
\alpha_{2}= & \frac{\sin \left(\theta-e_{\theta}\right)}{\frac{\phi}{L}}\left[g_{11} \frac{\phi}{L}\left(\omega \sin \theta-\left(\omega-e_{\omega}\right) \sin \left(\theta-e_{\theta}\right)\right)-\right. \\
& g_{12} \frac{\phi}{L}\left(\omega \cos \theta-\left(\omega-e_{\omega}\right) \cos \left(\theta-e_{\theta}\right)\right] \\
- & \frac{\cos \left(\theta-e_{\theta}\right)}{\frac{\phi}{L}}\left[g_{21} \frac{\phi}{L}\left(\omega \sin \theta-\left(\omega-e_{\omega}\right) \sin \left(\theta-e_{\theta}\right)\right)-\right. \\
& g_{22} \frac{\phi}{L}\left(\omega \cos \theta-\left(\omega-e_{\omega}\right) \cos \left(\theta-e_{\theta}\right)\right]
\end{aligned}
$$




$$
\left[\begin{array}{c}
\hat{x}_{d} \\
\hat{x}_{q}
\end{array}\right]=\left[\begin{array}{cc}
\cos \left(\theta-e_{\theta}\right) & \sin \left(\theta-e_{\theta}\right) \\
-\sin \left(\theta-e_{\theta}\right) & \cos \left(\theta-e_{\theta}\right)
\end{array}\right]\left[\begin{array}{cc}
\cos \theta & -\sin \theta \\
\sin \theta & \cos \theta
\end{array}\right]\left[\begin{array}{c}
x_{d} \\
x_{q}
\end{array}\right]
$$

Para garantizar el adecuado funcionamiento del sistema que tiene un observador en el lazo, es necesario estudiar la estabilidad del sistema dinámico formado por las ecuaciones (7.25)-(7.28) y (7.31)-(7.32). Esto es en general, un problema difícil. Sin embargo, daremos un argumento de plausibilidad utilizando la técnica de la perturbación singular [57], [58]. Ésta nos permitiría bajo ciertas suposiciones realizar un análisis separando la dinámica del observador y la del controlador. El sistema bajo estudio es de orden 6 , con vector de estado $\left[\begin{array}{llllll}\theta & \omega & x_{d} & x_{q} & e_{\theta} & e_{\omega}\end{array}\right]^{\prime}$. Partimos el vector de estado de la siguiente manera: $\zeta=\left[\begin{array}{llll}\theta & \omega & x_{d} & x_{q}\end{array}\right]^{\prime}$ y $\rho=\left[\begin{array}{ll}e_{\theta} & e_{\omega}\end{array}\right]^{\prime}$. Si elegimos $g=\max \left(g_{11}, g_{12}, g_{21}, g_{22}\right)$ y dividimos (7.31) y (7.32) por $g$, el sistema puede ser escrito de la siguiente forma:

$$
\begin{aligned}
\dot{\zeta} & =\sigma_{1}(\zeta, \rho, u) \\
\mu \dot{\rho} & =\sigma_{2}(\zeta, \rho, \mu)
\end{aligned}
$$

donde $\mu=1 / g$. Si se eligen los valores de $\left(g_{11}, g_{12}, g_{21}, g_{22}\right)$ para que el observador converja y $\max \left(g_{11}, g_{12}, g_{21}, g_{22}\right)$ sea grande y $\mu$ sea pequeño, entonces el subsistema (7.37) es de dinámica más rápida que el subsistema (7.36). Es posible satisfacer esta condición eligiendo $G$ como en el capítulo anterior (ver pág.100). Podemos rescatar el comportamiento de "baja frecuencia" si aproximamos el sistema dinámico por un sistema de orden 4 . En efecto, si $\mu=0$ la solución de $\sigma_{2}\left(\zeta, \rho_{0}, \mu\right)=0$ es $\rho_{0}=0$ y entonces $\varphi_{1}=\varphi_{2}=0$. Por lo tanto, es dado esperar que si el observador converge suficientemente "rápido", el comportamiento del sistema con observador sea el mismo que el del sistema sin observador.

Una demostración rigurosa puede basarse en mostrar que el sistema (7.36)-(7.37) satisface ciertas hipótesis (suposiciones 7.5.1 a 7.5.4 de [57]) y luego obtener estabilidad asintótica uniforme por la aplicación del teorema 5.1 del capítulo 7 de [57].

Nota: Con el criterio de diseño que estamos empleando parecería que los valores de $g_{11}$ y $g_{22}$ pueden ser tan grandes como se deseen. Sin embargo, en la imple- 
Figura 7.4: Cupla generada. Esquema de control sin observador

mentación ese valor estará limitado, pues las mediciones estarán contaminadas por ruido. Puede decirse, que el máximo valor $g$ debe ser compatible con el ruido de medición. Entonces una vez que decidimos el valor de $g$ la estabilidad del sistema empleando observador queda garantizada siempre que se pueda utilizar la técnica de perturbación singular.

\subsection{Resultados de simulación}

Como fue expresado oportunamente (ver 96) la cupla generada debe ser constante luego de superado el transitorio. En la figura 7.4 puede verse la forma de onda de la cupla generada cuando se utiliza un esquema de control sin observador. En este caso se realimenta la medición de posición para construir el esquema de control. A con- 
Figura 7.5: Esquema de control propuesto

tinuación ilustramos el comportamiento del conjunto planta-observador-controlador. En la figura 7.5 representamos el esquema de control propuesto que utiliza el observador y por lo tanto no necesita la medición de posición. El controlador usa la linealización exacta por transformación y relimentación y un controlador de velocidad PI (Proporcional + Integral) en el lazo externo junto a un controlador de corriente construído con la realimentación de los estado, pero empleando las variables estimadas $(\hat{\omega} \mathrm{y} \hat{\theta})$. Por lo tanto,

$$
\begin{aligned}
& v_{\alpha}=L\left(\cos \hat{\theta} u_{d}-\sin \hat{\theta} u_{q}\right)+R x_{\alpha}-L \hat{\omega} x_{\alpha}-\sin \hat{\theta} \phi \hat{\omega} \\
& v_{\beta}=L\left(\sin \hat{\theta} u_{d}+\cos \hat{\theta} u_{q}\right)+R x_{\beta}-L \hat{\omega} x_{\beta}+\cos \hat{\omega} \phi \hat{\omega} \\
& u_{d}=-K_{x_{d}} \hat{x}_{d}+r_{x_{d}}
\end{aligned}
$$




$$
u_{q}=-K_{x_{q}} \hat{x}_{q}+K_{p}\left(\omega^{\star}-\hat{\omega}\right)+K_{i} \int\left(\omega^{\star}-\hat{\omega}\right) d t
$$

donde $r_{x_{d}}$ es la referencia de corriente, $K_{p}$ es la ganancia proporcional, $K_{i}$ es la ganancia integral y $K_{x_{d}}$ y $K_{x_{q}}$ son las ganancias de realimentación. Los datos y los valores de parámetros del motor y del controlador son los dados en la siguiente tabla:

\begin{tabular}{||c|c||}
\hline$P_{N}$ & $600 \mathrm{~W}$ \\
$\Omega_{N}$ & $200 \mathrm{rad} / \mathrm{seg}$. \\
pares de polos & 1 \\
$L$ & $20.5 \mathrm{mHy}$ \\
$R$ & $1.55 \Omega$ \\
$\phi$ & $0.22 \mathrm{Nm} / \mathrm{A}$ \\
$J$ & $2.210^{-3} \mathrm{Kgm}^{2}$ \\
$B$ & $2.210^{-2} \mathrm{Nms} / \mathrm{rad}$ \\
$K_{x_{d}}$ & 10 \\
$K_{x_{q}}$ & 200 \\
$K_{p}$ & 1 \\
$K_{i}$ & 100 \\
$g_{11}$ & 500 \\
$g_{12}$ & 0 \\
$g_{21}$ & 0 \\
$g_{22}$ & 500 \\
\hline
\end{tabular}

La figura 7.6 muestra la respuesta del sistema cuando se sigue una referencia de velocidad $\left(\omega^{\star}\right)$ que varía de $50 \mathrm{rad} / \mathrm{seg}$ a $5 \mathrm{rad} / \mathrm{seg}$ y luego a $25 \mathrm{rad} / \mathrm{seg}$. Las condiciones iniciales en el observador son: $1 \mathrm{rad}$ para la posición estimada y $20 \mathrm{rad} / \mathrm{seg}$ para la velocidad estimada. Debido a las escalas de tiempo y la rápida convergencia del observador, la velocidad estimada, apenas puede distinguirse. Sin embargo, en la figura 7.7 hemos representado el error de velocidad $\left(e_{\omega}=\omega-\hat{\omega}\right)$ con la escala de tiempo ampliada donde puede verse, claramente, la convergencia. En la figura 7.8, 
Figura 7.6: Respuesta del sistema con observador 
Figura 7.7: Error de velocidad 
Figura 7.8: Error de posicion

se ilustra el error de posición. Finalmente presentamos la forma de onda de la cupla generada cuando se emplea observador (figura 7.9). Si se compara esta figura con la figura 7.4 se ve que luego de superado el transitorio de convergencia del observador ambas formas de onda son iguales como se muestra en la figura 7.10.

\subsection{Conclusión}

En este capítulo propusimos emplear el observador en un esquema de control de lazo cerrado que utiliza la linealización exacta por transformación y realimentación, para el control de velocidad del MSIP. Mediante este esquema pudimos eliminar los medidores de las variables mecánicas y los resultados obtenidos fueron altamente satisfactorios. Para garantizar un funcionamiento correcto del sistema es necesario 
Figura 7.9: Cupla generada. Esquema de control con observador 
Figura 7.10: Diferencia de Cupla 
elegir las ganancias del observador de manera que se pueda separar la dinámica de convergencia del mismo y la dinámica de la ley de control. Además mostramos como la transformación $D-Q$ aplicada al MSIP se relaciona con la ley obtenida para el control por linealización exacta por transformación y relimentación.

\section{Apéndice 7.A}

El problema de la linealización exacta por transformación y realimentación se encuentra bien tratado en [24], [47], [88]. Describiremos, brevemente, como puede hallarse la transformación empleada anteriormente. La notación en este apéndice es la usualmente utilizada por la mayoría de la bibliografía que trata este tema. Si denominamos $f$ y $g$ a dos campos vectoriales analíticos (o al menos suficientemente derivables), el corchete de Lie de $f(x)$ y $g(x)$ es denotado y definido como:

$$
[f, g]=\left(\frac{\partial g}{\partial x}\right) f-\left(\frac{\partial f}{\partial x}\right) g
$$

donde $\frac{\partial g}{\partial x}$ y $\frac{\partial f}{\partial x}$ son las matrices de derivadas parciales. La notación $a d_{f}^{i} g_{j}=$ $\left[f, a d_{f}^{i-1} g_{j}\right]$ para $i=1, \ldots$, con $a d_{f}^{0} g_{j}=g_{j}$, es usada para indicar sucesivas aplicaciones del corchete de Lie.

El modelo del MSIP es de la siguiente forma:

$$
\dot{\xi}=f(\xi)+u_{1} g_{1}+u_{2} g_{2}
$$

donde $\xi=\left[\begin{array}{llll}\theta & \omega & x_{\alpha} & x_{\beta}\end{array}\right]^{\prime} \in R^{4}, g_{1}$ y $g_{2}$ vectores campo constantes y $u_{1}$ y $u_{2}$ escalares. Se desea encontrar una transformación de estados:

$$
\begin{aligned}
& z_{1}=T_{1}(\xi) \\
& z_{2}=T_{2}(\xi) \\
& z_{3}=T_{3}(\xi) \\
& z_{4}=T_{4}(\xi)
\end{aligned}
$$

que permita llevar el sistema a la forma:

$$
\dot{z}_{1}=z_{2}
$$




$$
\begin{aligned}
& \dot{z}_{2}=z_{3} \\
& \dot{z}_{3}=\phi_{1}(z)+u_{1} \gamma_{11}(z)+u_{2} \gamma_{12}(z) \\
& \dot{z}_{4}=\phi_{2}(z)+u_{1} \gamma_{21}(z)+u_{2} \gamma_{22}(z)
\end{aligned}
$$

Derivando (7.43) a (7.46) con respecto al tiempo se obtiene:

$$
\begin{aligned}
& \dot{T}_{1}=\frac{\partial T_{1}}{\partial \xi} f(\xi)+u_{1} \frac{\partial T_{1}}{\partial \xi} g_{1}+u_{2} \frac{\partial T_{1}}{\partial \xi} g_{2} \\
& \dot{T}_{2}=\frac{\partial T_{2}}{\partial \xi} f(\xi)+u_{1} \frac{\partial T_{2}}{\partial \xi} g_{1}+u_{2} \frac{\partial T_{2}}{\partial \xi} g_{2} \\
& \dot{T}_{3}=\frac{\partial T_{3}}{\partial \xi} f(\xi)+u_{1} \frac{\partial T_{3}}{\partial \xi} g_{1}+u_{2} \frac{\partial T_{3}}{\partial \xi} g_{2} \\
& \dot{T}_{4}=\frac{\partial T_{4}}{\partial \xi} f(\xi)+u_{1} \frac{\partial T_{4}}{\partial \xi} g_{1}+u_{2} \frac{\partial T_{4}}{\partial \xi} g_{2}
\end{aligned}
$$

Por lo tanto, para satisfacer (7.47) se debe cumplir:

$$
\begin{aligned}
& \frac{\partial T_{1}}{\partial \xi} g_{1}=0 \\
& \frac{\partial T_{1}}{\partial \xi} g_{2}=0 \\
& \frac{\partial T_{2}}{\partial \xi} g_{1}=0 \\
& \frac{\partial T_{2}}{\partial \xi} g_{2}=0
\end{aligned}
$$

y para satisfacer las condiciones originales de controlabilidad [24], [47], [88] pediremos:

$$
\operatorname{det}\left[\begin{array}{cc}
\frac{\partial T_{3}}{\partial \xi} g_{1} & \frac{\partial T_{3}}{\partial \xi} g_{2} \\
\frac{\partial T_{4}}{\partial \xi} g_{1} & \frac{\partial T_{4}}{\partial \xi} g_{2}
\end{array}\right] \neq 0
$$

Aplicando la fórmula de Leibnitz [24], [47], [88] en (7.55) a (7.58) y (7.59), obtenemos:

$$
\begin{aligned}
& \frac{\partial T_{1}}{\partial \xi}\left[\begin{array}{llll}
g_{1} & a d_{f}^{1} g_{1} & g_{2} & a d_{f}^{1} g_{2}
\end{array}\right]=\left[\begin{array}{llll}
0 & 0 & 0 & 0
\end{array}\right] \\
& \operatorname{det}\left[\begin{array}{cc}
\frac{\partial T_{1}}{\partial \xi} a d_{f}^{2} g_{1} & \frac{\partial T_{1}}{\partial \xi} a d_{f}^{2} g_{2} \\
\frac{\partial T_{4}}{\partial \xi} g_{1} & \frac{\partial T_{4}}{\partial \xi} g_{2}
\end{array}\right] \neq 0
\end{aligned}
$$


Para el MSIP se tiene:

$$
f=\left[\begin{array}{c}
\omega \\
K_{1}\left(-x_{\alpha} \sin \theta+x_{\beta} \cos \theta\right)-K_{2} \omega \\
-K_{3} x_{\alpha}+K_{4} \omega \sin \theta \\
-K_{3} x_{\beta}-K_{4} \omega \cos \theta
\end{array}\right]
$$

donde $K_{1}=\frac{3 \phi}{2 J}, K_{2}=\frac{B}{J}, K_{3}=\frac{R}{L}$ y $K_{4}=\frac{\phi}{L}$.

$$
\begin{aligned}
& g_{1}=\left[\begin{array}{llll}
0 & 0 & K_{5} & 0
\end{array}\right]^{\prime} \\
& g_{2}=\left[\begin{array}{llll}
0 & 0 & 0 & K_{5}
\end{array}\right]^{\prime}
\end{aligned}
$$

donde $K_{5}=\frac{1}{L}$. Por lo tanto:

$$
a d_{f}^{1} g_{1}=-\Lambda\left[\begin{array}{c}
0 \\
0 \\
K_{5} \\
0
\end{array}\right]=\left[\begin{array}{c}
0 \\
K_{1} K_{5} \sin \theta \\
K_{3} K_{5} \\
0
\end{array}\right]
$$

donde

$$
\begin{gathered}
\Lambda=\left[\begin{array}{cccc}
0 & 1 & 0 & 0 \\
K_{1}\left(-x_{\alpha} \cos \theta-x_{\beta} \sin \theta\right) & -K_{2} & -K_{1} \sin \theta & K_{1} \cos \theta \\
-K_{4} \omega \cos \theta & K_{4} \sin \theta & -K_{3} & 0 \\
-K_{4} \omega \sin \theta & -K_{4} \cos \theta & 0 & -K_{3}
\end{array}\right] \\
a d_{f}^{2} g_{1}=\left[\begin{array}{c}
\partial a d_{f}^{1} g_{1} \\
\partial \xi-\frac{\partial f}{\partial \xi} a d_{f}^{1} g_{1} \\
-K_{1} K_{5} \sin \theta \\
K_{1} K_{5}\left(\omega \cos \theta+\left(K_{2}+K_{3}\right) \sin \theta\right. \\
-K_{4} K_{1} K_{5} \sin ^{2} \theta+K_{3}^{2} K_{5} \\
K_{4} K_{1} K_{5} \sin \theta \cos \theta
\end{array}\right]
\end{gathered}
$$




$$
\begin{aligned}
a d_{f}^{2} g_{2}= & \frac{\partial a d_{f}^{1} g_{2}}{\partial \xi} f-\frac{\partial f}{\partial \xi} a d_{f}^{1} g_{2} \\
= & {\left[\begin{array}{c}
K_{1} K_{5} \cos \theta \\
K_{1} K_{5}\left(\omega \sin \theta-\left(K_{2}+K_{3}\right) \cos \theta\right. \\
K_{4} K_{1} K_{5} \sin \theta \cos \theta \\
-K_{4} K_{1} K_{5} \cos ^{2} \theta-K_{3}^{2} K_{5}
\end{array}\right] }
\end{aligned}
$$

Por lo tanto de (7.60) obtenemos:

$$
\frac{\partial T_{1}}{\partial \xi}=\left[\begin{array}{llll}
\frac{\partial T_{1}}{\partial \theta} & 0 & 0 & 0
\end{array}\right]
$$

Por consiguiente la (7.61) es:

$$
\operatorname{det}\left[\begin{array}{cc}
\frac{\partial T_{1}}{\partial \theta} K_{1} K_{5} \sin \theta & \frac{\partial T_{1}}{\partial \theta} K_{1} K_{5} \cos \theta \\
\frac{\partial T_{4}}{\partial x_{\alpha}} K_{5} & \frac{\partial T_{4}}{\partial x_{\beta}} K_{5}
\end{array}\right] \neq 0
$$

Resolviendo (7.68),

$$
-K_{1} K_{5}^{2} \frac{\partial T_{1}}{\partial \theta}\left[\sin \theta \frac{\partial T_{4}}{\partial x_{\beta}}+\cos \theta \frac{\partial T_{4}}{\partial x_{\alpha}}\right] \neq 0
$$

Existen varias posibles soluciones para (7.69), por razones de "globalidad" elegimos una que hace el determinante constante. Por ello,

$$
\begin{aligned}
& z_{1}=\theta \\
& z_{2}=\omega \\
& z_{3}=K_{1}\left(-x_{\alpha} \sin \theta+x_{\beta} \cos \theta\right)-K_{2} \omega \\
& z_{4}=x_{\alpha} \cos \theta+x_{\beta} \sin \theta
\end{aligned}
$$

La relación con la transformación $D-Q$ es:

$$
\begin{aligned}
x_{d} & =z_{4} \\
x_{q} & =\left(z_{3}+K_{2} z_{2}\right) / K_{1} \\
\theta & =z_{1} \\
\omega & =z_{2}
\end{aligned}
$$


Las expresiones $(7.74-7.77)$ demuestran que la transformación $D-Q$ coincide con una transformación que linealiza exactamente el sistema por transformación y realimentación. 


\section{Capítulo 8}

\section{Conclusiones Finales}

\subsection{Resultados obtenidos}

En esta tesis hemos desarrollado observadores no lineales de orden reducido para ser empleados en accionamientos eléctricos, con el propósito de estimar la posición y la velocidad angular de los motores que forman parte de los mismos. Con el fin de satisfacer este objetivo desarrollamos las técnicas del capítulo 4 y las aplicamos al MRC (capítulo 5) y al MSIP (capítulo 6), representantes de las clases de los motores de reluctancia variable y de los motores de continua sin escobillas, respectivamente. Los resultados obtenidos permiten afirmar que es posible construir observadores cuya convergencia es "global" o "casi global" empleando técnicas no lineales. Es importante destacar que las demostraciones de convergencia tienen en cuenta que la señal de comando es función de la posición estimada. En el capítulo 7, hemos mostrado cómo se puede construir un esquema de control sin sensores mecánicos para un accionamiento de velocidad variable que utililiza un MSIP. En este esquema utilizamos el observador del capítulo 6 y la estrategia de linealización exacta por transformación y realimentación. Esto deja al accionamiento como un sistema lineal lo que permitió aplicarle una estrategia clásica $P I$.

Al comparar los observadores empleados para estimar la posición y la velocidad angular con los presentados por otros autores, podemos ver que de nuestra propuesta surgen las siguientes ventajas: 
- Una cota para la velocidad de convergencia puede ser obtenida a partir del método de diseño empleado.

- La convergencia no es una propiedad local cuando se satisfacen las condiciones de diseño.

- La capacidad de cómputo necesaria para implementar los observadores es menor que la que se necesita en la implementación de observadores de similar desempeño. Esto se debe a que los observadores son de orden reducido.

- Los observadores pueden emplearse en esquemas de control no lineal sin sensores mecánicos en los que se obtiene un muy buen desempeño que supera o iguala al presentado en otros trabajos.

\subsection{Sugerencias para futuros desarrollos}

Debe considerarse que los observadores están basados en disponer de un modelo correcto de la planta. En consecuencia, es muy importante conocer los parámetros del motor con un adecuado grado de exactitud. Algunas veces esto no es posible o los parámetros varían durante el funcionamiento del accionamiento, y por lo tanto puede considerarse la posibilidad de hacer los observadores adaptivos. Un primer paso en este sentido es el observador con estimador de cupla de carga del capítulo 5 . Pero está claro que un esquema de adaptación total implica la estimación de todos los parámetros. Por otro lado, los resultados obtenidos en el capítulo 6 permiten afirmar que se puede construir un observador que para estas aplicaciones es casi insensible a la variación de los parámetros mecánicos. Esto hace pensar que es posible intentar el diseño de observadores robustos; lo que abre un camino para posibles futuros desarrollos continuando la línea de investigación del presente trabajo. Por una parte, si se elige explorar la adaptación paramétrica se puede tratar de construir observadores cuyo error converja asintóticamente a cero. Pero debe considerarse que el número de ecuaciones diferenciales puestas en juego crece considerablemente, au- 
mentando la complejidad del estudio de la convergencia del sistema y el número de ecuaciones diferenciales a implementar. Esto también sucede si se usan esquemas adaptivos con las técnicas de estimación para velocidad y posición propuestas hasta el presente. Si se marcha hacia lo robusto, la simplificación de tener un número reducido de ecuaciones diferenciales se sigue manteniendo, pero es casi seguro que existirá un error de estimación permanente cuando los parámetros del modelo difieran de los del motor. El desafío consiste en construir un observador que permita garantizar que para todo posible valor de los parámetros, el error sea lo suficientemente pequeño como para que las estimaciones puedan emplearse en un esquema de control sin producir un grave deterioro en el desempeño del mismo.

Podemos manifestar que la aplicación de las técnicas del capítulo 4 puede resultar ampliamente provechosa en otros problemas de estimación. Por ejemplo, dentro del campo de la electrónica de potencia, se pueden utilizar para estimar el flujo en accionamientos que emplean el motor de inducción. Esta estimación es necesaria si se desea construir un accionamiento de velocidad variable usando la técnica del control vectorial. Otro aspecto a considerar es el establecimiento de la óptima configuración de hardware que permite la más eficiente implementación de las técnicas propuestas.

Un problema interesante surge debido al empleo del PWM en el comando de accionamientos. Por lo general, como hicimos en esta tesis, suelen emplearse métodos de promediación ('averaging') [100], [101] para estudiar el problema que aparece debido a la discontinuidad del lado derecho de las ecuaciones diferenciales. La discontinuidad es generada por el modelo usado para representar el PWM. En ese sentido, un camino a seguir es el de considerar las inclusiones diferenciales [112], [123] como un marco teórico para el estudio del citado problema. Sin embargo, no debe perderse de vista que la implementación física del sistema no permite que tales discontinuidades existan más allá del modelo utilizado.

Finalmente, consideramos que puede resultar interesante analizar esquemas de control que empleen observadores y además consideren las restricciones que provienen del empleo de actuadores que limitan los valores de las señales de comando como 
puede verse en [107] y también que restringen el valor de los estados [75]. 


\section{Bibliografía}

[1] B.D.O. Anderson and J.B. Moore, Optimal Filtering, Prentice-Hall, New Jersey, USA, 1979.

[2] K. Åström and B. Wittenmark, Computer controlled systems, Prentice-Hall, New Jersey, USA, 1984.

[3] D.J. Atkinson, P.P. Acarnley and J.W. Finch, "Observer for induction motor state and parameter estimation", IEEE Trans. on Ind. Appl., Vol. 27, No. 6, pp. 1119-1127, Nov./Dec. 1991.

[4] A. Bado, S. Bolognani and M. Zigliotto, "Effective Estimation of Speed and Rotor Position of a PM Synchronous Motor Drive by a Kalman Filtering Technique", Proc. of the Power Electronics Specialists Conference (PESC'92), Toledo, Spain, pp. 951-957, Jun. 1992.

[5] W.T. Baumann and W.J. Rugh, "Feedback control of nonlinear systems by extended linearization", IEEE Trans. on Aut. Cont., Vol. 31, No. 1, pp. 40-45, 1986.

[6] A. Bellini, G. Figalli and T. Lombari, "An approach to induction machines by means of the bilinear system theory", Proc. Int. Conf. Electrical Machines, Brussels, Belgium, pp. E2/2, 1978.

[7] A. Bellini, G. Figalli and G. Ulivi, "Realization of a bilinear observer of the induction machine", Proc. 2nd. Int. Conf. Electrical Variable Speed Drives, London, England, pp. 175-178, 1979. 
[8] D. Bestle and M. Zeitz, "Canonical form observer design for non-linear timevariable systems", Int. J. Control, Vol. 38, No. 2, pp. 419-431, Aug. 1983.

[9] J. Birk and M. Zeitz, "Extended Luenberger observer for non-linear multivariable systems", Int. J. Control, Vol. 47, No. 6, pp. 1823-1836, Jun. 1988.

[10] B. Blake, "New applications and developments in switched reluctance drives", Proc. of the Conference on Drives/Motors/Controls, Birmingham, U.K., pp. 4.19-4.24, 1988.

[11] J. Böcker and J. Janning, "Discrete-Time flux observer for PWM inverter fed induction motors", Proc. of the European Power Electronics (EPE'91), Firenze, Italy, Sep. 1991.

[12] M. Bodson, J. Chiasson, R. Novotnak and R. Rekowski, "High-performance nonlinear feedback control of a permanent magnet stepper motor", IEEE Trans. on Contr. Syst. Tech., Vol. 1, No. 1, pp. 1-13, Mar. 1993.

[13] S. Bolognani, G. Buja and M. I. Valla, "Switched-Reluctance motor performance analysis based on an improved modeling of its magnetic characteristics", Electric Machines and Power Systems, Vol.19 , No.4, pp. 542-547, 1991.

[14] B. Bose and P. Szczesny, "A microcomputer-based control and simulation of an advanced IPM synchronous machine drive system for electric vehicle propulsion", IEEE Trans. on Ind. Elect., Vol. 35, No. 4, pp. 547-559, Nov. 1988.

[15] J. Brewer, "Kronecker products and matrix calculus in system theory", IEEE Trans. on Circuits and Systems, Vol. cas-25, No. 9, pp. 772-781, Sep. 1978.

[16] G. Buja, R. Menis and M.I. Valla, "Variable Structure Control of a SRM Drive", IEEE Trans. on Industrial Electronics, Vol. 40, No. 1, pp. 56-63, Feb. 1993. 
[17] G. Buja, R. Menis and M.I. Valla, "Disturbance torque estimation in a sensorless DC drive", Proc. of the IEEE Ind. Elect. Conference (IECON'93), Hawaii, USA, pp. 977-981, Sep. 1993.

[18] C. Canudas de Wit, N. Fixot and K.J. Åström, "Trajectory tracking in robot manipulators via nonlinear estimated state feedback", IEEE Trans. on Robotics and Aut., Vol. 8, No. 1, pp. 138-144, Feb. 1992.

[19] C. Chen, Linear system theory and design, CBS College Publishing, New York, USA, 1984.

[20] N. Chen, "Exact terminal control of switched reluctance motors by feedback linearization", Proc. of the ISMM International Symposium, Hawaii, USA, pp. 208-211, Feb. 1988.

[21] J.N. Chiasson and R.T. Novotnak, "Nonlinear speed observer for PM stepper motor", IEEE Trans. on Aut. Contr., Vol. 38, No. 10, pp. 1584-1588, Oct. 1993.

[22] E. Coddington and N. Levinson, Theory of ordinary differential equations, Mc.Graw-Hill, 1955.

[23] D.G. Cumming, "Design of observers of reduced dynamics", Electron. Lett., 5, No. 10, pp. 213-214, May 1969.

[24] C. D’Attelis, Introducción a los sistemas no lineales de control y sus aplicaciones, Asociación Argentina de Control Automático, Bs. As., Argentina, 1992.

[25] R. Dhaouadi, N. Mohan and L. Norum, "Design and Implementation of an Extended Kalman Filter for the State Estimation of a Permanent Magnet Synchronous Motor", IEEE Trans. on Power Electronics, Vol. 6, No. 3, pp. 491-497, Jul. 1991. 
[26] A. Ellison, Conversión electromecánica de la energía, Librería de las Naciones, Bs. As., Argentina, 1969.

[27] C. Elmas and H. Zelaya-De La Parra, "Position sensorless operation of a switched reluctance drive based on observer", Proceedings of the European Power Electronics Association, Brighton, England, pp. 82-87, Sep. 1993.

[28] S.V. Emelyanov, Titles in new types of feedback, variable structure systems and binary control, International Research Institute for Management Sciences, Moscow 1990.

[29] N. Ertugrul and P. Acarnley, "A new algorithm for sensorless operation of permanent magnet motors", IEEE Trans. on Ind. Appl., Vol. 30, No. 1, pp. 126-133, Jan./Feb. 1994.

[30] M. Etchechoury, J. Solsona, C. Muravchik, "Sistemas no lineales con control que incluyen un observador para su linealización por realimentación", Anales del X Congreso Chileno de Ingeniería Eléctrica, Valdivia, Chile, Oct. 1993, pp. E-31-E-36.

[31] A. Ferrero and A. Raciti, "A digital method for the determination of the magnetic characteristics of variable reluctance motors", IEEE Trans. on Instr. and Meas., Vol. 39, No. 4, 604-608, Aug. 1990.

[32] A. Fitzgerald and C. Kingsley, Electric Machinery, Mc Graw-Hill, USA, USA, 1961

[33] A. Fratta, A. Vagati and F. Villata, "On the evolution of ac machines for spindle drive applications", IEEE Trans. on Ind. Appl., Vol. 28, No. 5, pp. 1081-1086, 1992.

[34] T. Furuhashi, S. Sangwongwanich and S. Okuma, "A position-and-velocity sensorless control for brushless DC motors using an adaptive sliding mode observer", IEEE Trans. on Ind. Elec., Vol. 39, No. 2, pp. 89-95, Apr. 1992. 
[35] R. García, "Algunos aspectos de la Teoría del Control de Sistemas no Lineales: Observadores de tipo Luenberger", Tesis Doctoral, Fac. de Ciencias Exactas y Naturales, UBA, Argentina, 1993.

[36] A. Gelb, Applied optimal estimation, The M.I.T. Press, USA, 1974.

[37] B. Gopinath, "On the control of linear multiple input-output systems", Bell Syst. Tech. J., 50, pp. 1063-1081, Mar. 1971.

[38] H. Gutt, "Trends in developing electrical actuators", European Trans. on Electrical Power Engineering, Vol. 2, No. 2, pp. 69-76, 1992.

[39] W. Hahn, Stability of motion, Springer-Verlag, USA, 1967.

[40] M.H. Haque, "Estimation of three-phase induction motor parameters", Electric power systems research, 26, pp. 187-193, 1993.

[41] H. Hashimoto, V. Utkin, J.X. Xu, H. Suzuki and F. Harashima, "VSS observer for linear time varying system", Proc. of the IEEE Industrial Electronics Conference (IECON'90), pp. 34-39, Pacific Grove, USA, Nov. 1990.

[42] H. Henderson and S. Searle, "Vec and vech for matrices, with some uses in Jacobians and multivariable statistics", The Canadian Journal of Statistics, Vol. 7, No. 1, pp. 65-81, 1979.

[43] R. Hermann and A. Krener, "Nonlinear controllability and observability", IEEE Trans. on Aut. Contr., Vol. AC-22, No. 5, pp. 728-740, Oct. 1977.

[44] J. Hindmarsh, Máquinas eléctricas y sus aplicaciones, Ed. Urmo S. A., Bilbao, España, 1974.

[45] J. Holtz, "Pulsewidth modulation - A survey", IEEE Trans. on Ind. Elect., Vol. 39, No. 5, pp. 410-420, Oct. 1992. 
[46] M. Ilic-Spong, R. Marino, S. Peresada and D. Taylor, "Feedback Linearizing Control of Switched Reluctance Motors", IEEE Trans. Aut. Contr., Vol. AC32, No. 5, pp. 371-379, May 1987.

[47] A. Isidori, Nonlinear Control Systems. An Introduction, 2nd. Edition, Springer-Verlag, Berlin, Germany, 1989.

[48] T. Iwasaki and T. Kataoka, "Application of an extended Kalman filter to parameter identification of an induction motor", Proc. of the IEEE Ind. Appl. Soc. Annual Meeting, (IAS'89), San Diego, USA, pp. 248-253, Oct. 1989.

[49] A. Jazwinski, Stochastic processes and filtering theory, Academic Press, Inc., New York, USA, 1970.

[50] L. Jones and J. Lang, "A State Observer for the Permanent-Magnet Synchronous Motor", IEEE Trans. on Ind. Elec., Vol. 36, No. 3, pp. 374-382, Aug. 1989.

[51] T. Kailath, Linear Systems, Prentice Hall Inc., New York, 1980.

[52] K. Kamiyama, K. Saito, I. Shimizu, K. Imagawa, M. Tobise and Y. Watanabe, "The state-of-the-art rolling mill motor drives in Japan", Proceedings of the 6th. Conference on Power Electronics and Motion Control, Budapest, Hungary, pp. 13-17, Oct. 1990.

[53] R.E. Kalman, "A new approach to linear filtering and prediction problems", Trans. ASME, Vol. 82D, pp.35, 1960.

[54] R.E. Kalman and R. Bucy, "New results in linear filtering and prediction", Trans. ASME, Vol. 83D, p.95, 1961.

[55] H. Keller, "Non-linear observer design by transformation into generalized observer canonical form", Int. J. Control, Vol. 46, No. 6, pp. 1915-1930, Dec. 1987. 
[56] T. Kenjo, Stepping motors and their microprocessor controls, Clarendon Press, Oxford, 1984.

[57] P. Kokotovic, H. Khalil and J. O'Reilly, Singular perturbation methods in control: Analisys and Design, Academic Press, Florida, USA, 1986.

[58] P. Kokotovic and H. Khalil (Edited by), Singular perturbations in systems and control, IEEE Press, New York, USA, 1986.

[59] S. Kou, D. Elliot and T. Tarn, "Exponentials Observers for Nonlinear Dynamic Systems", Information and Control, Vol. 29, pp. 204-216, 1975.

[60] A.J. Krener and A. Isidori, "Linearization by output injection and nonlinear observers", Systems \& Control Letters, 3, pp. 47-52, Jun. 1983.

[61] A. Krener and W. Respondek, "Nonlinear observers with linealizable error dynamics", SIAM J. Control and Optimization, Vol. 23, No. 2, pp. 197-216, Mar. 1985.

[62] H. Kwakernaak and R. Sivan, Linear optimal control systems, John Wiley \& Sons, Inc., Canada, 1972.

[63] J.H. Lang and G.C. Verghese, "Adaptive Estimation of States and Parameters in Electrical Machines", Proc. of the IEEE Ind. Elect. Conference (IECON'90), pp. C1.1-C1.7, Pacific Grove, USA, Sep. 1990.

[64] D. Lawrence, "On a nonlinear observer with pseudo-linearized error dynamics", Proc. of the 31st Conference on Decision and Control (CDC'92), Tucson, Arizona, USA, pp. 751-756, Dec. 1992.

[65] P. Lawrenson, J. Stephenson, P. Blenkinsop, J. Corda and N. Fulton, "Variable-speed switched reluctance motors", IEE Proceedings Pt. B, Vol. 127, No. 4, pp. 253-265, Jul. 1980. 
[66] S. Lefschetz, Differential Equations: Geometric Theory, Second Edition, Dover Publications, Inc., New York, 1977.

[67] W. Leonhard, "High-performance digital motion control with electrical servo drives", Proc. of the 6th. Conference on Power Electronics and Motion Control, Budapest, Hungary, pp. 1-12, Oct. 1990.

[68] C.W. Li and L. W. Tao, "Observing non-linear time-variable systems through a canonical form observer", Int. J. Control, Vol. 44, No. 6, pp. 1703-1713, Dec. 1986.

[69] T. Lipo, "Recent progress in the development of solid-state AC motor drives", IEEE Trans. on Power Electronics, Vol. PE-3, No.2, pp. 105-117, Apr. 1988.

[70] T. Low, T. Lee and K. Chang, "A Nonlinear Speed Observer for PermanentMagnet Synchronous Motor", IEEE Trans. on Ind. Elec., Vol. 40, No. 3, pp. 307-315, Jun. 1993.

[71] D. G. Luenberger, "Observers of low dynamic order", IEEE Trans. Military Electr., Vol. 3, No. 2, pp. 74-80, 1964.

[72] D.G. Luenberger, "An introduction to observers", IEEE Trans. Aut. Contr., Vol. AC-16, pp. 596-603, Dec. 1971.

[73] A. Lumsdaine and J. Lang, "State Observers for Variable-Reluctance Motors", IEEE Trans. Ind. Elect., Vol. 37, No. 2, pp. 133-142, Apr. 1990.

[74] J. Lyons, S. Mac Minn and M. Preston, "Flux/Current Methods For SRM Rotor Position Estimation", Conference Record of the 1991 IEEE Industry Applications Society Annual Meeting, Vol. I, pp. 482-487, Jun. 1991.

[75] J. Mancilla Aguilar, R. García, C. D’Attellis, "Linealización exacta de sistemas no lineales: Seguimiento de trayectorias con controles limitados y restricciones 
de estado", Anales del XIV Simposio Nacional de Control Automático, Bs. As., Argentina, pp. 165-170, Sep. 1994.

[76] N. Matsui and M. Shigyo, "Brushless de motor control without position and speed sensors", IEEE Trans. on Ind. Appl., Vol. 28, No. 1, pp. 120-127, Jan./Feb. 1992.

[77] R. Marino, "Adaptive observer for single output nonlinear systems", IEEE Trans. on Aut. Contr., Vol. 35, No. 9, pp. 1054-1058, Sep. 1990.

[78] T. Miller, Switched Reluctance Motor Drive. A Reference Book of Collected Papers, Intertec Communications Inc., CA(USA), 1988.

[79] T.J.E. Miller, Brushless Permanent-Magnet and Reluctance Motor Drives, Clarendon Press, Oxford, 1989.

[80] T. Miller, J. Stephenson, S. MacMinn and J. Hendershot, "Switched Reluctance Drives", Tutorial presented at the 1990 IEEE Industry Applications Soc. Annual Meeting, (IAS'90), Seattle, USA, Oct. 1990.

[81] H. Moghbelli, G. Adams and R. Hoft, "Performance of a 10-Hp switched reluctance motor and comparison with induction motors", IEEE Trans. on Ind. Appl., Vol. 27, No. 3, pp. 531-538, May/Jun. 1991.

[82] N. Mohan, T. Undeland and W. Robbins, Power Electronics: Converters, Applications and Design, John Wiley \& Sons, Inc., Singapore, 1989.

[83] J. Moreira, "Torque Ripple Minimization in Switched Reluctance Motors Via Bi-Cubic Spline Interpolation", Proc. of the IEEE Power Electronic Specialists (PESC'92), pp. 851-856, 1992.

[84] S. Morimoto, K. Hatanaka, Y. Tong and Y. Takeda, "Servo drive system and control characteristics of salient pole permanent magnet synchronous motor", IEEE Trans. on Ind. Appl., Vol. 29, No. 2, pp. 338-343, Mar./Apr. 1993. 
[85] J. Murphy and F. Turnbull, Power Electronic Control of AC Motors, Pergamon Press, UK, 1988.

[86] I. Nagrath and M. Gopal, Control systems engineering, 2nd. Edition, John Wiley and Sons, Inc., USA, 1982.

[87] P.K. Nandam and P.C. Sen, "A comparative study of a Luenberger observer and adaptive observer-based variable structure speed control system using a self-controlled synchronuos motor", IEEE Trans. on Ind. Electr., Vol. 37, No. 2, pp. 127-132, Apr. 1990.

[88] H. Nijmeijer and A.J. van der Schaft, Nonlinear Dynamical Control Systems, Springer-Verlag, New York, Inc., USA, 1990.

[89] R. Nilsen and M.P. Kazmierkowski, "Reduced-order observer with parameter adaption for fast rotor flux estimation in induction machines", IEE Proceedings, Vol. 136, Pt.D, No. 1, pp. 35-43, Jan. 1989.

[90] R. Nilsen and M.P. Kazmierkowski, "New reduced-order observer with parameter adaption for flux estimation in induction motors", Proc. of the IEEE Power Electronic Specialists (PESC'92), pp. 245-252, 1992.

[91] T. Orlwska-Kowalska, "Application of extended Luenberger observer for flux and rotor time-constant estimation in induction motor drives", IEE Proceedings, Vol. 136, Pt.D, No. 6, pp. 324-330, Nov. 1989.

[92] S. K. Panda and G.A.J. Amaratunga, "Switched reluctance motor drive without direct rotor position sensing", Proc. IEEE Ind. Appl. Soc. Annual Meeting (IAS'90), Seattle, USA, pp. 525-530, Oct. 1990.

[93] A. Phelps, "On constructing nonlinear observers", SIAM J. Control and Optimization, Vol. 29, No. 3, pp. 516-534, May 1991. 
[94] P. Pillay and R. Krishman, "Application characteristics of permanent magnet synchronous motor and brushless dc motors for servo drives", IEEE Trans. on Ind. Appl., Vol. 27, No. 5, pp. 986-996, Sep./Oct. 1991.

[95] W. Press, B. Flannery, S. Teukolsky and W. Vetterling, Numerical Recipes. The Art of Scientific Computing, Cambridge University Press, USA, 1986.

[96] A. Radun, "High-power density switched reluctance motor drive for aerospace applications", IEEE Trans. on Ind. Appl., Vol. 28, No. 1, pp. 113-119, Jan./Feb. 1992.

[97] W.F. Ray and I.H. Al-Bahadly, "Sensorless methods for determining the rotor position of switched reluctance motors", Proc. of the European Power Electronics Association, Brighton, England, pp. 7-13, Jun. 1993.

[98] C. Reboulet and C. Champetier, "A new method for linearizing nonlinear systems: the pseudolinearization", Int. J. Cont., Vol. 40, No. 4, pp. 631, Oct. 1984.

[99] R. Sanchez Peña, Introducción a la Teoría de Control Robusto, Asociación Argentina de Control Automático, Bs. As., Argentina, 1992.

[100] S. Sanders, J. Noworolski, X. Liu and G. Verghese, "Generalized averaging method for power conversion circuits", IEEE Trans. on Power Electronics, Vol. 6, No. 2, pp. 251-259, Apr. 1991.

[101] S. Sanders and G. Verghese, "Synthesis of averaged circuit models for switched power converters", IEEE Trans. on Circuits and Systems, Vol. 38, No. 8, pp. 905-915, Aug. 1991.

[102] S. Sangwongwanich, T. Yonemoto, T. Furuhashi and S. Okuma, "Design of sliding observer for robust estimation of rotor flux of induction motors", Int Power Electronics Conference, Tokyo, Japan, pp. 1235-1242, 1990. 
[103] P. Sen, "Electric motor drives and control - Past, present and future", Proc. of IEEE Industrial Electronics Conference (IECON'88), Singapore, pp. 534-544, 1988.

[104] R.B. Sepe and J. Lang, "Real-Time observer-based (adaptive) control of a permanent-magnet synchronuos motor without mechanical sensors", IEEE Trans. on Ind. Appl., Vol. 28, No. 6, pp. 1345-1352, Nov./Dec. 1992.

[105] J.J E. Slotine, J.K Hedrick and E. A. Misawa, "On Sliding Observers for Nonlinear Systems", Journal of Dynamic Systems, Measurement, and Control, Vol. 109, pp. 245-252, Sep. 1987.

[106] J. Solsona, D. Kleiman, C. Muravchik, "Observadores con dinámica lineal del error para sistemas no lineales de control", Anales de la 4ta. Reunión de Trabajo en Procesamiento de la Información y Control (RPIC'91), pp. 493500, Nov. 1991.

[107] J. Solsona, M. Etchechoury, C. Muravchik, "Sobre la linealización exacta de sistemas con saturación", Anales del XIII Simposio Nacional de Control Automático, Bs. As., Argentina, pp. 84-89, Sep. 1992.

[108] J. Solsona, M. Etchechoury, M.I. Valla, C. Muravchik, "Un observador no lineal de orden reducido para motores de reluctancia conmutada", Anales del X Congreso Chileno de Ingeniería Eléctrica pp. E.107-E.112, Valdivia, Chile, Oct. 1993.

[109] J. Solsona, M.I. Valla, M. Etchechoury, C. Muravchik, "Estimación de la posición y la velocidad angular del motor sincrónico de imán permanente", Anales del XIV Simposio Nacional de Control Automático, pp. 335-340, Bs. As., Argentina, Sep. 1994.

[110] J.J. Slotine and W. Li, Applied Nonlinear Control, Prentice-Hall, Inc., New Jersey, USA, 1991. 
[111] A. Frank D'Souza, Design of Control Systems, Prentice-Hall, Inc. New Jersey, USA, 1988.

[112] P. Tallos, "Viability problems for nonautonomous differential inclusions", SIAM J. Control and Optimization, Vol. 29, No. 2, pp. 253-263, Mar. 1991.

[113] D.G. Taylor, "An Experimental Study on Composite Control of Switched Reluctance Motors", IEEE Control Systems Magazine, Vol. 11, No. 2, pp.31-36, Feb. 1991.

[114] F.E. Thau, "Observing the state of nonlinear dynamic systems", Int. J. Control, Vol. 18, pp. 471-479, 1973.

[115] V. Utkin, "Variable structure systems with sliding modes", IEEE Trans. on Aut. Contr., Vol. AC-22, No. 2, pp. 212-222, Feb. 1977.

[116] M.I. Valla, "Control de Motores de Reluctancia Conmutada", Tesis Doctoral, Fac. de Ingeniería, UNLP, mayo 1994.

[117] A. J. van der Schaft, "On nonlinear observers", IEEE Trans. on Aut. Contr., Vol. 30, No. 12, pp. 1254-1256, Dec. 1985.

[118] G.C. Verghese and S.R. Sanders, "Flux estimation in induction machines", IEEE Trans. on Ind. Elect., Vol. 35, No. 1, pp. 85-94, Feb. 1988.

[119] M. Vidyasagar, Nonlinear systems analysis, Prentice-Hall, USA, 1978

[120] B.L. Walcott, M.J. Corless and S.H. Zak, "Comparative study of non-linear state-observation techniques", Int. J. Control, Vol. 45, No. 6, pp. 2109-2132, Jun. 1987.

[121] B.L. Walcott and S.H. Zak, "State Observation of Nonlinear Uncertain Dynamical Systems", IEEE Trans. on Aut. Cont., Vol. AC-32, No. 2, pp. 166-169, Feb. 1987. 
[122] R. Wallace and D. Taylor, "Low-torque ripple switched reluctance motors for direct-drive robotics", IEEE Trans on Robotics and Automation, Vol. 7, No. 6, pp. 733-742, Dec. 1991.

[123] P. Wolensky, "A uniqueness theorem for differential inclusions", Journal of Differential Equations, Vol. 84, pp. 165-182, Mar. 1990.

[124] X.H. Xia and W.B. Gao, "On exponential observers for nonlinear systems", Systems \& Control Letters, Vol. 11, No. 3, pp. 319-325, 1988.

[125] X.H. Xia and W.B. Gao, "Nonlinear observer design by observer error linearization", SIAM J. Control and Optimization, Vol. 27, No. 1, pp. 199-216, Jan. 1989.

[126] J. Xu, H. Hashimoto and F. Harashima, "On Design of VSS Observer for Nonlinear Systems", Trans. of the Society of Instrument and Control Engineers, Vol. 25, No. 2, pp. 152-158, Feb. 1989.

[127] J. Xu, and H. Hashimoto, "Parameter identification methodologies based on variable structure control", Int. J. Control, Vol. 57, No. 5, 1207-1220, May 1993.

[128] S. Zak, "On Stabilization and Observation of Nonlinear/ Uncertain Dynamic Systems", IEEE Trans. on Aut. Cont., Vol. 35, No. 5, pp. 604-607, May 1990. 